\title{
Testing the transtheoretical model of behavior change : validity and applicability for fruit intake
}

Citation for published version (APA):

de Vet, E. W. M. L. (2005). Testing the transtheoretical model of behavior change : validity and applicability for fruit intake. [Doctoral Thesis, Maastricht University]. Kurt Lewin Instituut. https://doi.org/10.26481/dis.20051216ev

Document status and date:

Published: 01/01/2005

DOI:

10.26481/dis.20051216ev

Document Version:

Publisher's PDF, also known as Version of record

\section{Please check the document version of this publication:}

- A submitted manuscript is the version of the article upon submission and before peer-review. There can be important differences between the submitted version and the official published version of record.

People interested in the research are advised to contact the author for the final version of the publication, or visit the DOI to the publisher's website.

- The final author version and the galley proof are versions of the publication after peer review.

- The final published version features the final layout of the paper including the volume, issue and page numbers.

Link to publication

\footnotetext{
General rights rights.

- You may freely distribute the URL identifying the publication in the public portal. please follow below link for the End User Agreement:

www.umlib.nl/taverne-license

Take down policy

If you believe that this document breaches copyright please contact us at:

repository@maastrichtuniversity.nl

providing details and we will investigate your claim.
}

Copyright and moral rights for the publications made accessible in the public portal are retained by the authors and/or other copyright owners and it is a condition of accessing publications that users recognise and abide by the legal requirements associated with these

- Users may download and print one copy of any publication from the public portal for the purpose of private study or research.

- You may not further distribute the material or use it for any profit-making activity or commercial gain

If the publication is distributed under the terms of Article $25 \mathrm{fa}$ of the Dutch Copyright Act, indicated by the "Taverne" license above, 


\section{Testing the Transtheoretical model of behavior change}

Validity and applicability for fruit intake 



\title{
Testing the Transtheoretical model of behavior change
}

\author{
Validity and applicability for fruit intake
}

\section{PROEFSCHRIFT}

ter verkrijging van de graad van doctor aan de Universiteit Maastricht, op gezag van de Rector Magnificus, Prof. mr. G.P.M.F. Mols volgens het besluit van het College van Decanen, in het openbaar te verdedigen op vrijdag 16 december 2005 om 11.45 uur

door

Emily Wilhelmina Maria Lucia de Vet 


\section{Promotores:}

Prof dr.ir. J. Brug

Prof dr. N.K. de Vries

\section{Copromotor:}

Dr. J. de Nooijer

\section{Beoordelingscommissie:}

Prof. dr. H. de Vries (voorzitter)

Prof dr. G.J. Kok

Dr. S.P.J. Kremers

Prof. dr. D.T.D de Ridder (Universiteit Utrecht)

Prof. dr. N.D. Weinstein (Rutgers University, United States of America)

Cover design by Ellen Oosterwijk

Printed by Optima Grafische Communicatie

ISBN 90-76269-48-3

The studies described in this thesis were performed at the Nutrition and Toxicology Research Institute Maastricht (NUTRIM), which participates in the Graduate School VLAG (Food Tedunology, Agrobiotechnology, Nutrition and Health Sciences) and at the Kurt Lewin Institute. Both institutes are accredited by the Royal Netherlands Academy of Arts and Sciences.

The studies were conducted with financiall support from the Netherlands Organisation for Health Research and Development (ZonMW, project number 4005.0003) 
Voor ons ma 


\section{Contents}

General Introduction

\section{Part 1 Predictors of stage transitions}

Chapter 1 Determinants of forward stage transitions: a Delphi study

Chapter 2 Determinants of forward stage transition for precontemplation and contemplation for fruit consumption

Chapter 3 Do the Transtheoretical processes of change predict transitions in stages of change for fruit intake?

\section{Part 2 Staging health behavior change}

Chapter 4 Stages of change in fruit intake: a longitudinal examination of stability, stage transitions and transition profiles

Chapter 5 The Transtheoretical stages of change and fruit intake: real or pseudostages?

Chapter 6 Testing the Transtheoretical model for fruit intake: comparing web-based individualized stage-matched and stage-mismatched feedback

General Discussion

References

Summary \& Samenvatting 


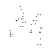

thes a ? +

...

$\therefore<\div$

$-$ 


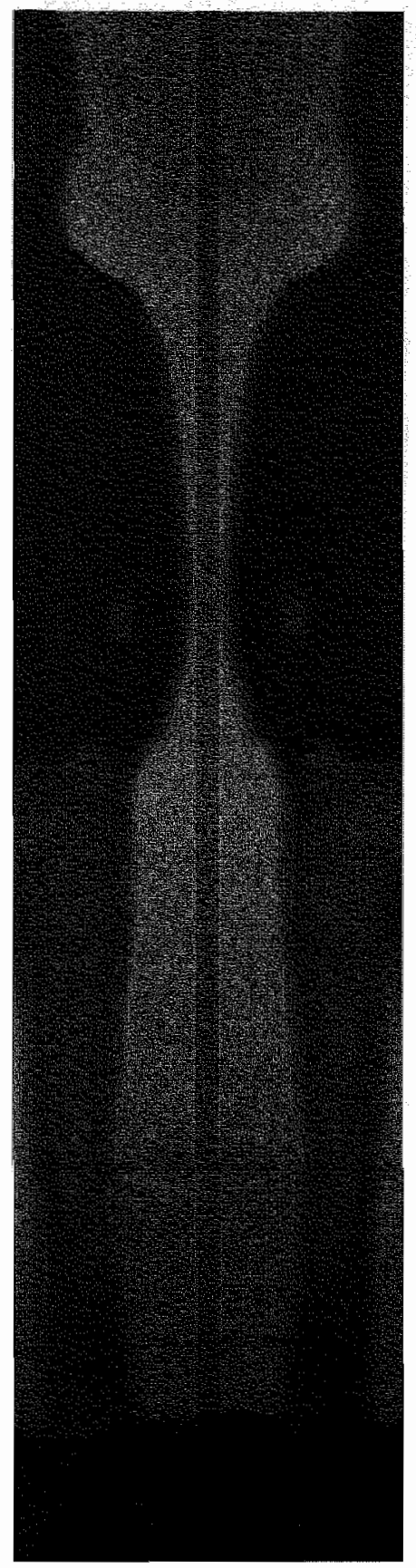

General Introduction 


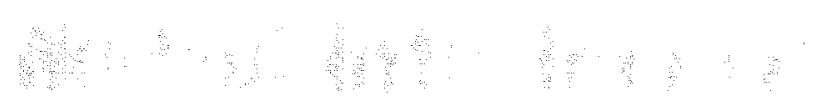




\section{General introduction}

Chronic diseases, such as cardiovascular diseases and cancers, are major causes of death in most Western countries, including the Netherlands (Statistics Netherlands, 2003). For many of these diseases, behavioral factors are associated with an increased risk, and healthy lifestyles can reduce the risk of acquiring such diseases (e.g., Van der Wilk \& Jansen, 2005; Van Oers, 2002; WHO/FAO, 2003). Health education is a discipline aiming to promote such healthy lifestyles, by means of planned activities that stimulate learning through communication to facilitate voluntary actions conducive to health (Green \& Kreuter, 1999). These health education activities are most likely to achieve health behavior change, when activities are guided by social and behavioral science theory (Kok, Schaalma, De Vries, Parcel, \& Paulussen, 1996; Kok, Schaalma, Ruiter, Van Empelen, \& Brug, 2004; Kok, Van Den Borne, \& Dolan Mullen, 1997).

Theories that are used frequently in health education are multi-stage models (e.g, the Transtheoretical model; Prochaska \& DiClemente, 1983). Multistage models conceptualize health behavior change as a process comprising several discrete stages, and delineate processes that are involved with establishing motivation, facilitating behavioral enaction and providing maintenance strategies (Armitage \& Conner, 2000).

Such models can be used in two forms of theory-based research, i.e., problem-driven and theory-driven research (Kok et al., 1996). In problem-driven research, insights from different relevant theories are used to understand and solve a particular problem, whereas in theory-driven research a specific theory is tested in an applied setting in order to gain insight into the validity of the theory (Brug, Oenema, \& Ferreira, 2005; Kok et al., 1996). The studies in this thesis are of the latter kind. More specifically, predictions and assumptions of the Transtheoretical model of behavior change will be systematically tested when the model is applied to fruit intake.

\section{The Transtheoretical model of behavior change}

The Transtheoretical model (TTM) of behavior change originated from research on how people change addictive behaviors with and without psychotherapy. The model conceptualizes that behavior change unfolds through a series of stages of change (Prochaska \& DiClemente, 1983). The TTM concentrates on intentional change and considers change as a dynamic process that involves movement 
through a series of five discrete, qualitatively different stages of change. Precontemplation is the stage in which people are not intending to take action in the foreseeable future, usually defined as the next six months. People in the contemplation stage are intending to change in the next six months. In preparation, people intend to take action in the immediate future, usually defined as the next month. Action is the stage in which people have made specific overt modifications in their behavior somewhere in the past six months (e.g., quit smoking). When a behavior change persists for more than six months, the maintenance stage is reached (Prochaska \& Velicer, 1997a). In addition, a termination stage was defined in which people are no longer at risk for relapse to earlier stages. However, a majority of people may never reach this ideal stage ${ }_{r}$ and termination is nowadays hardly ever presented as part of the TTM (Prochaska \& Velicer, 1997a).

According to the TT'M, behavior change is not a linear process, but people tend to cycle through the stages; they can progress as well as regress from one stage to another. Such movements between stages of change are called stage transitions. People can regress (or relapse) to all earlier stages of change, though, when people progress, it is assumed that individuals will pass through all the stages as outlined by the TTM and stage skipping is not expected (Prochaska, DiClemente, Velicer, \& Rossi, 1992; Prochaska, Redding, \& Evers, 2002).

These stages of change are used to integrate principles and processes derived from different leading theories of intervention and behavior change, hence the name Transtheoretical (Prochaska \& DiClemente, 1983). The TTM integrates the stages of change concept with other components, i.e., decisional balance, self-efficacy, and the processes of change. Decisional balance is based on Janis and Mann's model of decision making (Janis \& Mann, 1977) and refers to an individual's relative weighing of pros and cons (Prochaska et al., 1994). To progress from precontemplation to contemplation the pros of changing must increase, while the cons of changing must decrease for progress from contemplation. According to the TTM, a crossover should occur, usually between contemplation and preparation, after which the pros will outweigh the cons (Prochaska et al., 1994).

Self-efficacy is derived from Bandura's self-efficacy theory (Bandura, 1982) and reflects the situation-specific confidence people have that they can cope with high-risk situations without relapsing. The TTM assumes that self-efficacy increases monotonically from precontemplation to maintenance (Velicer, DiClemente, Rossi, \& Prochaska, 1990). Originally, the TTM included a temptation component, which reflects the intensity of urges to engage in a specific habit when in the midst of difficult situations. The TTM posits that, in contrast to self-efficacy, temptation tends to decline monotonically across the stages of change. Temptations seem to reflect the converse of self-efficacy. Therefore, 
research based on the TTM includes either self-efficacy or temptation (Velicer; Prochaska, Fava, Norman, \& Redding, 1998).

The processes of change represent the overt and covert activities that people use to progress from stage to stage. Therefore, the processes of change should guide intervention development. Ten processes have been identified. These ten processes are grouped into the experiential processes (cognitive, affective and evaluative processes) and behavioral processes (active strategies). The experiential processes should be used to progress from early stages, whereas behavioral processes should become important in later stages of change (Prochaska et all., 2002). Decisional balance, self-efficacy and processes of change will be introduced in depth in chapters 2 and 3.

Each stage of change requires a different set of tasks and the specific TTM components should be applied to facilitate progress to further stages of change. The TTM outlines several consequences of a staging perspective on health behavior change interventions. First, interventions should be designed to meet the needs of individuals at each of the stages of change, so-called stage-matched, stage-based or stage-tailored interventions. Second, interventions should not be evaluated solely in terms of efficacy, but also in terms of impact. Traditional action-oriented interventions mainly target preparators, while only a small percentage of the at-risk population may actually be ready for change. The impact of such interventions may therefore be low. In addition to the matching of interventions to stage of change, proactive recruitment techniques (actively reaching out to interact with all potential participants) are needed to ensure that also precontemplators and contemplators are reached with interventions. Third, the TTM argues that the amount of progress is directly related to the stage individuals were in at the start of the interventions (the so-called stage-effect). It will take more time for precontemplators to progress to action than for preparators. Therefore, forward stage transition should be used as an outcome measure in addition to or instead of actual behavior change as the indicator of intervention success (Prochaska, DiClemente, \& Norcross, 2003; Prochaska et al., 2002; Prochaska \& Velicer, 1997a).

Probably due to its intuitively appealing framework, the TTM has gained great popularity over the last 20 years. The model is now widely applied to study determinants of health behaviors, to design interventions and to evaluate the efficacy and impact of such interventions. The original context of addictive behaviors has been expanded and now includles acquisition behaviors (e.g., smoking initiation), screening behaviors (mammograplyy screening), mental health problems (e.g., eating disorders), HIV prevention (e.g., condom use), medication compliance, and exercise and dietary behaviors (Prochaska et al,, 2002). 


\section{Application of the Transtheoretical model to dietary behaviors}

The TTM has also been applied to dietary behaviors, which are more or less continuous in nature. The TTM has most often been applied to fat, fruit and vegetable intake (see for example Greene \& Rossi, 1998; Ling \& Horwath, 2000; Ma, Betts, \& Horacek, 2001; Van Duyn et al, 2001), but also to grain, fiber and fish consumption (e.g, Ling \& Horwath, 2000; Nitzke et al,, 1999; Siero, Broer. Bemelmans, \& Meyboom-de Jong, 2000).

The application of the TIM to such continuous behaviors deserves further attention, since it might be different from the application to more dichotomous addictive behaviors. One such difference concerns the goal of behavior change. For smoking cessation, for instance, the goal is to abstain completely, while eating a healthy diet could be described as a more general behavior or behavioral category (Povey, Conner, Sparks, James, \& Shepherd, 1999). For example, reducing fat intake involves several behavioral actions such as food purchase, food preparation, or portion size control. Further, although a diet may have been altered in a healthy way (such as a reduction of fat intake) that does not mean that the diet meets the recommendations for a healthy diet (Ni Mhurchu, Margetts, \& Speller, 1997). A second difference concerns individuals" perception of their own consumption levels. Several studies showed that there might be a discrepancy between more objective dietary assessments (such as food frequency questionnaires) and completely subjective self-rated dietary intake (e.g. Lechner, Brug, \& De Vries, 1997; Lechner, Brug, de Vries, Van Assema, \& Mudde, 1998). Third, differences might exist between dietary change and smoking cessation in maintaining desired change (Bowen, Meischke, \& Tomoyasu, 1994). Compared to maintaining smoking cessation, maintaining dietary change requires a contimued effort, in which the changes need to be performed frequently (Bowen et al., 1994). Further, a concept related to maintenance, i.e., relapse, may have a different meaning for dietary change than for smoking cessation. Relapse describes a return to an unhealthy habit. In smoking cessation, relapse is defined as smoking more than 20 cigarettes after a period of 24 hours of not smoking. Bowen (1994) suggests that for dietary change people 'relapse' when they realize they have gradually slipped away from the desired consumption levels.

There is a need to test assumptions and predictions from the TTM to gain more insight into the validity of the TTM, as will be elaborated upon further in the next subsection. Taking into account the differences between dietary behaviors and addictive behaviors from which the TTM originated, it seems particularly useful to test the validity and applicability of the TTM for dietary behavior. This thesis will test the TTM when applied to fruit intake. Four arguments exist for testing the TTM's validity as applied to fruit intake. First, fruit intake is a dietary behavior that is recommended on a daily basis, and may 
therefore be suitable to study stage transitions and the factors that relate to them as well as changes in consumption. Second, research has shown that misconception of intake levels occurred less frequently for fruit intake compared to other dietary behaviors (Lechner et al., 1998). Third, the TTM is a model of intentional behavior change. Fruit intake might be more intentionally controlled than, for example, vegetable or fat intake. Vegetables and foods contributing to total fat intake might be more frequently consumed during meals and in family settings and might therefore be under less individual control. Fourth, ample fruit intake may contribute to the prevention of obesity (Rolls, Ello-Martin, \& Carlton Tohill, 2004), and cardiovascular diseases (Srinath Reddy \& Katan, 2004). Ample fruit intake may also reduce the risk of cancers of the oesophagus, stomach and head and neck area (Dutch Cancer Society, 2004). In most countries, including the US and most countries in Europe, a majority of people does not meet the guidelines for sufficient fruit intake (Serdula et al., 2004; Van der Wilk \& Jansen, 2005 ). In the Netherlands, approximately $70 \%$ of the population eat less than the recommended minimum amount of two servings ( 250 grams) of fruit per day (Dutch Nutrition Centre, 1998; Van Oers, 2002)

\section{Validity of the Transtheoretical model}

The TTM is a widely applied model. However, debate exists about the model's validity, not only for the application of the TTM to diet. Concerns have been expressed that the stages of change are not real stages, but might reflect arbitrary pseudostages, i.e., segments of an underlying continuum (Bandura, 1998; Sutton, 2001; Weinstein, Rothman, \& Sutton, 1998). Furthermore, the TTM has been criticized for the definition of stages of change, the ordering of stages, the qualitative difference of stages of change, and on matching of interventions to stages of change (Bandura, 1997, 1998; Brug et al., 2005; Davidson, 1998; Littell \& Girvin, 2002; Sutton, 2000a; Sutton, 2001; Weinstein et al., 1998). These main critiques will be discussed in the light of stage model properties as proposed by Weinstein and colleagues (1998). These properties should discern a sound stage model from a pseudostage or continuum model of behavior change.

A first property of stage theories is a classification system to define stages. A stage theory needs a set of rules that assign each individual to one of a limited number of categories (Weinstein et al., 1998). If individuals are in more than one stage at a specific moment in time, this would disconfirm the discreteness of stages of change. Individuals can be assigned to stages using multi-dimensional questionnaires. Each stage is measured by a set of questionnaire items and scores are derived for each individual representing their position on each dimension. Such scaling instruments have indeed revealed strong correlations between adjacent stages and even between non-adjacent 
stages, and it has been argued that these results are actually evidence against discrete stages of change (Sutton, 2001). The first property can be (somewhat artificially) achieved by measuring stage of change with an algorithm for which

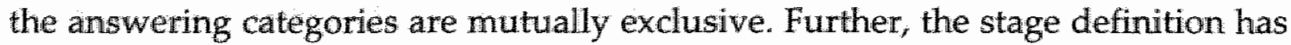
been criticized for the time frame. It seems that there is no theoretical reasoning or empirical evidence that would indicate that the six-month time frame is an appropriate basis for defining the stages (Kraft, Sutton, \& McCreath-Reynolds, 1999). The onlly argument that can be extracted from the literature is that six months is about as far in the future as most people plan a specific behavior (Velicer, Hughes, Fava, \& Prochaska, 1995). Moreover, changing the time frames would lead to different stage distributions (Sutton, 2000a), and as a consequence individuals would receive different interventions.

The second property of a sound stage theory is a specified sequence of stages through which a substantial majority of people move to lasting behavior change (Weinstein et al., 1998). Bandura $(1997,1998)$ argued that in a valid stage theory the sequence of stages should be invariant (a pupa cannot become a butterfly without first being a caterpillar) and irreversible (a butterfly cannot revert to a caterpillar): everyone moves through the same sequence and only forward transitions to the next stage are allowed. In his view, behavior change stages are descriptive categories of intention. Shifts from one such category to another will not make behavior change a dynamic process following discrete, qualitatively different stages (Bandura, 1998). Others have argued that Bandura's strict assumptions with invariant and irreversible stages are appropriate for biological or developmental stages, but may be unrealistic for stages of behavior change for which the process of change can be halted, reversed, or even abandoned at any point (Sutton, 2001; Weinstein et al., 1998).

The third property is that people at a given stage face similar barriers and consequently that they can be helped by similar interventions (Weinstein et al., 1998). The stages should represent relatively homogenous groups. However, research has shown large within-stage differences and stages have been subdivided into several other stages (e.g., Dijkstra, Bakker, \& de Vries, 1997; Kremers, Mudde, \& De Vries, 2001; Norman, Velicer, Fava, \& Prochaska, 2000).

The fourth property is that people in different stages face different barriers, i.e., some barriers must be more important at certain stages than others. Different interventions are needed to help people progress through the stages (Weinstein et al., 1998). Following from the third and fourth property, stages of change should be qualitatively different. Bandura (1998) argued that people just vary in their self-regulatory command as they fluctuate in their struggle to exercise control over their health behaviors, but that does not mean that qualitative transformations occur. Furthermore, even if certain factors would differ qualitatively between genuine stages, the TTM does not clearly specify the factors that influence transitions from one stage to the next (Sutton, 2001).

In their responses to these criticisms, TTM proponents have claimed that many misinterpretations and misapplications of the TTM exist which result in 
misleading conclusions (Padula et al., 2003; Prochaska \& DiClemente, 1998; Prochaska et al., 1992; Prochaska \& Velicer, 1997b). For example, Prochaska and Velicer (1997b) argue that stage is a variable and stage should not be equated with theory (a theory would after all include the systematic relationships between variables). Similarly, Prochaska and DiClemente (1998) have argued that it is critical for the evaluation of the stages of change and other components of the model that the application of the constructs is done as accurately as possible. The processes of change, for example, are usually omitted from the model, while these processes are proposed to be the independent variables within the TTM.

\section{Testing stage models}

It is important to note that almost all comments on the TTM result from a lack of well-designed studies testing the TTM's predictions for a concrete behavior, whereas problem-driven studies applying (parts of) the TTM abound. Weinstein and coworkers (1998) suggested three types of research designs that could be used to test predictions and assumptions from stage models. First, cross-sectional differences in variables between stages of change should show non-linear (discontinuity) patterns. This is the approach most often used to study the TTM. However, cross-sectional comparisons do not provide strong support for stage theories or for the alternative continuum theories (Weinstein et al., 1998). Second, longitudinal designs should be employed. On the one hand, longitudinal data can be used to test the property that people move through stages in the sequence hypothesized. On the other hand, prospective studies can test the assumption that different factors predict different stage transitions. Third, experimental designs can provide stronger evidence for stage theories. Two experimental designs can be employed, a so-called match-mismatch test and a sequence test. If certain factors influence transitions from one stage (e.g., precontemplation), and other variables influence transitions from another stage (e.g., contemplation), then interventions matched to the stage and stage-specific predictors of change should be more effective than interventions mismatched to the stage (i.e., matched to a different stage of change). Briefly, in precontemplators it should be more effective to target variables that influence forward transition from precontemplation than to target variables that influence forward transition from contemplation, and vice versa for contemplators. This is a match-mismatch test. Next, a sequence test can be conducted. Stage models hypothesize that the sequence of interventions should follow the hypothesized sequence of stages. To exemplify, for precontemplators it should be more effective to start with a precontemplationmatched intervention followed by a contemplation-matched intervention compared to the reverse order of intervention delivery. 


\section{Outline of the thesis}

There is a need to test assumptions and predictions from the TTM to gain more insight into the validity of the TTM. In this thesis, studies are presented that systematically test these assumptions and predictions for fruit intake using study designs necessary for stage model testing.

The first part of the thesis is concerned with the qualitative difference of stages of change, by studying predictors of stage transitions. Chapter 1 presents the results from a three-round Delphi study. By means of a Delphi methodology we attempted to integrate existing views on stage transition determinants among stages of change researchers and to identify the extent to which researchers agreed on these determinants. Next, the results from a longitudinal cohort study with six measurements in a four-month period are clescribed. In chapters 2 and 3 , results from the analyses of three assessments in this longitudinal cohort study are presented. Chapter 2 examines whether pros, cons, self-efficacy and fruit intake differ between stages of change at three points in time (cross-sectional comparisons) and whether these same variables predict forward stage transition out of precontemplation and contemplation for two periods of time (longitudinal analyses). Chapter 3 examines whether the processes of change predict stage transitions, and whether experiential processes are indeed of importance in early stages and behavioral strategies in later stages as outlined by the TTM.

The second part of the thesis examines whether health behavior change is adequately described by stages of change. Chapter 4 explores the stability of stages of change, patterns of stage transitions, and the sequences within these patterns during the six assessments of the longitudinal cohort study. Chapter 5 examines whether the Transtheoretical pre-action stages of change represent discrete categories or a continuous variable that is categorized into stage-like categories, so-called pseudostages using three assessments of the longitudinal cohort study. In chapter 6 the results of a match-mismatch test are presented, in which we tested in precontemplators and contemplators the effects of web-based individualized precontemplation-matched, contemplation-matched or actionmatched fruit promotion feedback.

The results are discussed and integrated in the General Discussion. Further, methodological and theoretical issues are discussed in that chapter, as well as implications for future research and practice. 


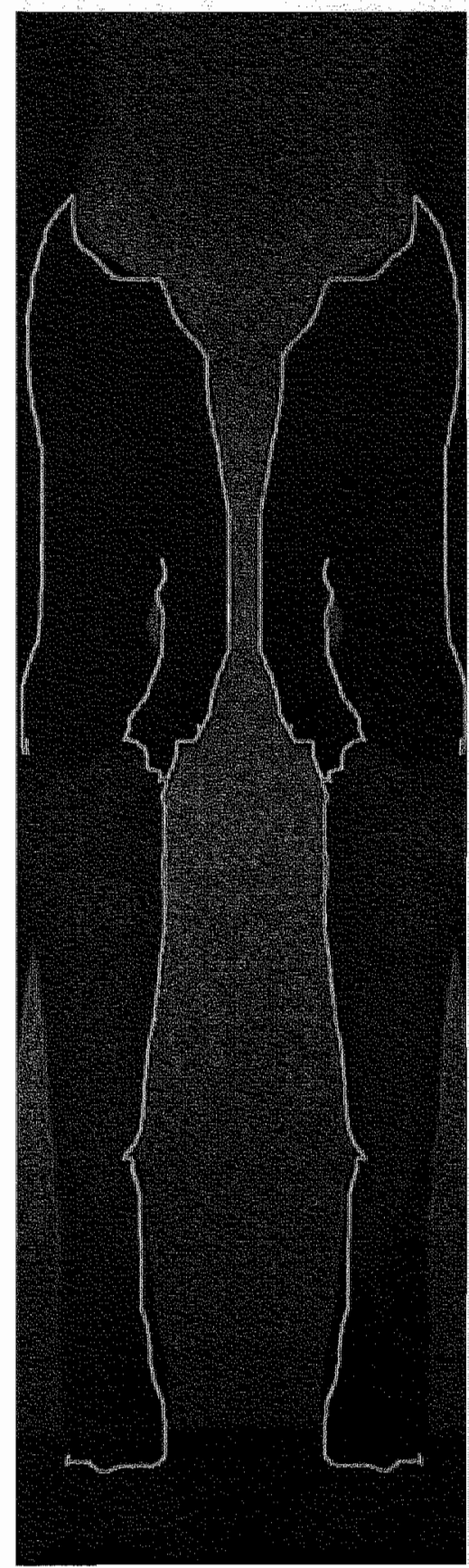

\section{Part 1 \\ Predictors of stage transitions}


$\therefore \quad \therefore$ 


\title{
Chapter 1 \\ Determinants of forward stage transitions: \\ a Delphi study
}

\begin{abstract}
Insight into stage transition determinants is necessary to develop and evaluate stage-tailored health promoting interventions. A three round Delphi study among stages of change researchers was conducted to make an inventory of opinions and examine agreement on determinants of forward transitions between Transtheoretical stages of change. In the first round, ten experts completed an electronic questionnaire with open-ended questions about potential determinants for each stage transition. In the second round, a structured electronic questionnaire based on the first round results, was sent to authors of scientific papers on stages of change, published between 1995 and May 2002. In the third round, participants were presented feedback about the second round and were asked to re-rate their answers based on the information provided. Results showed that participants agreed on various transition determinants, but that determinants were not always stage-specific, e.g., control-related issues and social support were identified as determinants of all transitions from contemplation to maintenance. The results further showed lower consensus about determinants of earlier stage transitions than about determinants of later stage transitions. The Delphi study identified hypotheses worthy of further examination in longitudinal observational and experimental studies.
\end{abstract}

Published as: De Vet, E., Brug, J., De Nooijer, J., Dijkstra, A., \& De Vries, N. K. (2005). Determinants of forward stage transitions: a Delphi study. Health Education Research, 20, 195-205. 


\section{Introduction}

The stages of change concept from the Transtheoretical model (TTM) (Prochaska, DiClemente, \& Norcross, 1992) has been widely used as a basis for planning health promotion interventions (e.g., Naylor, Simmonds, Riddoch, Velleman, \& Turton, 1999; Prochaska, DiClemente, Velicer, \& Rossi, 1993), as an indicator for effects of health promotion interventions (e.g., Brug \& Van Assema, 2000; Resnicow, McCarty, \& Baranowski, 2003) and to examine determinants of health behavior change (e.g., Brug, Glanz, \& Kok, 1997; Ma et al., 2002; Plotnikoff, Hotz, Birkett, \& Courneya, 2001). The TTM construes behavior change as a dynamic process involving progress through a series of five so-called Stages of Change: precontemplation (no intention to change behavior within the next six months), contemplation (intention to change behavior within the next six months), preparation (intention to change behavior within 30 days), action (people changed from unhealthy to healthy behavior within the past six months), and maintenance (maintenance of the behavior change for more than six months). According to the TTM, people can move forward and backward through the stages of change, but none of the stages will be skipped.

The proposed staged character of behavior change has important implications for research and practice in the area of health education and promotion, since stage theories imply that a central goal for health education and promotion should be to promote forward transitions through the stages to attain lasting behavior change. This can be achieved by matching health promotion interventions to stage of change through targeting those variables that can induce forward transitions through the stages (Redding et al., 1999). To adequately develop interventions matched to stage of change, information about these variables or forward transition determinants is needed.

The Transtheoretical model suggests some of these determinants, such as self-efficacy, decisional balance, processes of change and temptation (Prochaska \& Velicer, 1997a), and a large number of other alternative potential determinants have been suggested in the literature, e.g., attitude, subjective norm, social support, intention, affect, awareness, comparison to others, commitment to act, dispositional risk factors, vulnerability (see Brug et al., 1997; Courneya, Plotnikoff, Hotz, \& Birkett, 2001; De Vries \& Mudde, 1998; Ronda, Van Assema, \& Brug, 2001; Santi, Cargo, Brown, Best, \& Cameron, 1994; Segan, Borland, \& Greenwood, 2002; Van Duyn et al., 2001).

In the past two decades, a wide range of research and practice based on the Transtheoretical. Model in many different health-related domains has been executed. Integration of these findings and perspectives is necessary to gain overview in what we have learned about determinants of forward transitions so far, and how this knowledge can be used to improve health promotion practice. 
By means of a Delphi methodology, the present study aimed to provide such an overview by (a) making an inventory among stages of change researchers of opinions on forward stage transition determinants, and (b) identifying the extent to which participants agree on these determinants. In the discussion section the results of the present study will be compared to findings from longitudinal studies.

Although forward stage transition determinants might vary by behavior, the present study did not focus on one specific behavior, since it attempted to integrate concepts and perspectives into an overall systematic inventory on stage transition determinants.

\section{Delphi methodology versus systematic literature review}

Several authors have underlined the usefulness of Delphi methodology in health (promotion) research (De Meyrick, 2003; Green \& Kreuter, 1999; Jones \& Hunter, 1995; McKenna, 1994). As De Meyrick (2003, p.7) phrased it "the Delphi method is particularly well suited to health issues. On many important health issues, there is a relatively small group of acknowledged experts whose knowledge is the only real guide to best practice in relation to the issue of concern." This usefulness of the Delphi technique originates in its central characteristics (a) anonymity, protecting the Delphi results from the influences of group conformity, prestige, power and politics; (b) iteration; Delphi procedure taking place over a number of rounds, allowing individuals to change their opinion; (c) controlled feedback; between rounds, results of the previous round are communicated, and (d) statistical group response; a device to assure that the opinion of every member of the group is equally represented in the final response (Jones \& Hunter, 1995; Linstone \& Turoff, 1975; Pill, 1971; Rowe, Wright, \& Bolger, 1991). The Delphi method. enables working with a variety of target group representatives and large numbers of participants can be handled. Further, a Delphi methodology can be helpful in situations where face-to-face discussions are impractical (Green \& Kreuter, 1999).

One might argue that for an integration of findings and perspectives on stage transition determinants a systematic literature review or meta-analysis would be the most appropriate research method. However, since the designs used in stages of change research differ greatly, a comparison of these studies would be difficult. For example, longitudinal designs provide stronger tests of stage transition determinants, but not many have been conducted to date. Further, most studies evaluate stage transition determinants based on a singletheory perspective and therefore only examine a few concepts in relation to stage transition. The Delphi methodology provides the opportunity to derive from a multiple-theory perspective without the limitations of a systematic review or meta-analysis. 


\section{Method}

A three round Delphi study was conducted assessing two sorts of agreement. First, the extent to which each expert individually agrees with a statement that the factor under consideration determines stage transition. We refer to this sort of agreement as "agreement with stage transition determinan", representing the individual ratings of a potential stage transition determinant. Second, the extent to which experts collectively agree on the potential stage transition determinant under consideration. We refer to this sort of agreement as "consensus" representing the distribution of opinions of all experts. This distinction is made since the main interest of the present study was to identify determinants that experts collectively rate as an important predictor of stage transition, although it is possible that participants collectively agree (small distribution) that the factor under consideration does not determine stage transition (low rating).

All questionnaires of the three-round Delphi study were pretested among experts in the field of health promotion research.

\section{First round}

\section{Procedures and Participants}

Research and practice into staging behavior change varies greatly in e.g., health topic studied, the theoretical framework used, study methods, and materials developed. Since the first round was mainly used to generate a list of possible transition determinants and diversity was of importance, the first round experts had to be representative for the variety in research and practice. Therefore, a heterogeneous sample of experts from different protessional fields was recruited (health promotion, health education, marketing, psychotherapy etc.). These experts all had experience with theory-based research or practice into staging behavior change. An invitation letter to participate in all three rounds of the Delphi study, together with the first round questionnaire, was sent by email to a selection of 18 experts on stages of change. The experts were asked to complete the questionnaire and retum it by email. They were also offered the opportunity to complete the questionnaire by telephone. Two weeks after the questionnaires were sent out, non-responders received a reminder. Ten experts completed the first round interview, nine by email and one by telephone. Six experts were not able or willing to complete the interview, and two did agree to participate but did not complete the first round questionnaire in time to be included in the development of the questionnaire for the second round. 


\section{Questionnaire}

An electronic questionnaire consisting of nine open-ended questions was used in the first round, asking about potential predictors of stage transition. For each stage transition, experts were asked to name factors that predicted the stage transition, e.g., "Which factors do you think predict contemplation of behavioral change among people with no initial intention to change?" The first round questionnaires were primarily conducted to provide input for the second round questionnaire.

\section{Data analyses}

For each question, all answers were written down in a list. Answers that described the same variable were gathered into one item. Three researchers did this separately and differences in interpretations were discussed. The analysis resulted in a list of possible predictors of stage transitions ordered by stage.

\section{Second round}

\section{Procedures and participants}

The second round sample consisted of authors of scientific papers published between 1995 and May 2002, with stages of change in the title or keywords, which were identified through a literature search in PsychInfo and Medline. This search revealed 272 publications. Since some people were first author on more than one publication, the study population consisted of 235 first authors of whom 186 correct email addresses could be retrieved. If the first author"s email address could not be identified, the second author's address was tried. In total, 203 persons were invited to participate by an email letter including a link to an electronic questionnaire. Two reminders were sent, two and five weeks after the invitation email letter. The second reminder also included a question to examine non-response. In total 77 respondents ( $38 \%$ response rate) completed the questionnaire, including 11 who were not first authors and eight respondents from the first round.

\section{Questionnaire}

For the second round a structured 77-item electronic questionnaire was developed, based on the possible determinants identified by the experts in the first round (see http:/www.gvo.unimaas.nl/test/delphi.asp). Twenty-one items represented potential determinants of the transition from precontemplation to contemplation. Twenty-five items operationalized possible determinants of the transition from contemplation to preparation. Ten and 17 items represented possible determinants of the transition from preparation to action and from action stage to maintenance, respectively. For each stage transition an extra item "other, 


\section{Chapter 1}

namely..." was included, so participants could suggest determinants not put forward by the first round participants and therefore not included in the questionnaire. Items were presented in alphabetical order. For each stage transition, participants were asked to rate on a 7-point Likert scale, ranging from 3 (fully disagree) to 3 (fully agree), to what extent they agreed with statements about possible predictors and correlates of the relevant stage transition. Examples of items are: "I think that transition from the precontemplation stage to the contemplation stage is associated with anticipated regret about not behaving in a healthy/precautionary way" or "I think that transition from the preparation stage to the action stage is associated with self-efficacy to behave in a healthy/precautionary way". For all items an "I don't know" answer option was included.

\section{Data analyses}

Delphi studies generally use median scores to summarize the first sort of agreement, i.e., agreement with a statement. A median score represents the value below and above which half the cases fall, the $50^{\text {th }}$ percentile. The second sort of agreement, consensus, is generally calculated by using interquartile deviations (Jones \& Hunter, 1995). Interquartile deviation (IQD) represents the distance between the $25^{\text {th }}$ percentile and the $75^{\text {th }}$ percentile values in opinions, with a smaller IQD indicating larger consensus. An IQD $\leq 1$ can be considered as good consensus on a 7-point Likert scale (Linstone \& Turoff, 1975). An IQD $\leq 1$ means that more than $50 \%$ of all opinions falls within one point on the scale. In the present study, for each item median ratings and IQDs were calculated to assess the extent to which respondents collectively agreed with the stage transition determinant under consideration.

\section{Third round}

\section{Procedures and participants}

The third round sample $(\mathrm{N}=73)$ consisted of all respondents of the second round, provided that they had indicated they were willing to participate further by entering their name or email address in the second round questionnaire. After 3,5 weeks a reminder was sent. Forty-nine respondents completed the questionnaire (24\% response rate, $67 \%$ of second round participants), of which five respondents
were first round participants.

\section{Questionnaire}

In the third round, the second round questionnaire with feedback on the second round group results (median and IQD) was presented to participants. According
to the Delphi methodology ${ }_{x}$ items on which consensus was obtained in the second 
round (IQD $\leq 1$ ) were excluded from the third round questionnaire (see http://www.gvo.unimaas.nl/test/delphi3.asp).

The questionnaire consisted of 55 items. Participants were asked to complete the questionnaire and to re-rate their answers based on the information provided.

\section{Data analyses}

In the third round, group median ratings and IQDs were again analyzed. For each of the four stage transitions, changes in consensus between the second and third round were assessed using Wilcoxon Signed Rank Tests. Differences in IQDs between the second and third round were computed for each item within a stage transition. Next, these differences were ranked and positive and negative ranks were summed. When the sum of positive ranks is significantly different from the sum of the negative ranks, there is a change in consensus for that particular stage transition. All statistical analyses were conducted using SPSS 11.0.

\section{Results}

\section{First round}

As said, the first round questionnaire was primarily conducted to provide input for the second round questionnaire. Therefore, the second round questionnaire represents the first round group results as depicted in the items in Table 1.1.

\section{Second round}

Table 1.1 shows the results of the second round. In this second round, consensus was obtained on $18 \mathrm{items}(\mathrm{IQD} \leq 1)$. For 8 of these $18 \mathrm{items}$ also agreement $(M d n=$ 3) was established. All eight items were either in the transition from preparation to action (issues related to self-efficacy such as actual skills, commitment to act, overcoming practical barriers and self-efficacy) or in the transition from action to maintenance (issues related to (perceived) control such as coping skills to resist temptations to revert to unhealthy/risky behavior, self-efficacy to maintain healthy/precautionary behavior and skills to cope with difficult situations that may hinder healthy/precautionary behavior, and automatism such as the extent to which behaving in a healthy/precautionary way has become habitual). In total 15 of the 18 items on which consensus was obtained had a median score higher or equal to two, representing the "strongly agree with the item" opinion. 
Table 1.1

Results of the Delphi study per item (second and third round)

Second round

Third round

Hem

N Mdn IQD N Mdn IOD

\section{A. Transition from the precontemplation stage to contemplation stage}

1. Anticipated regret about not behaving in a

$69-1 \quad 3$

healthy/precautionary way

2. Awareness of the personal relevance of changing unhealthy/risky behavior

3. Awareness of the relation between unhealthy risky behavior and health problems*

4. Awareness that you are behaving in an unhealthy/risky way

5. Beliefs concerning the effectiveness of healthy/ precautionary behavior in preventing health problems

6. Cognitive attitudes towards healthyl precautionary behavior

7. Cues to action to perform to behave in a healthy/ precautionary way

8. Emotions involved with healthy/precautionary behavior

9. Emotions involved with unhealthy/risky behavior

10. Experience with people with a symptom or the condition for which healthy/precautionary behavior is recommended

11. Experiencing a symptom that might be related to unhealthy/risky behavior

12. Knowledge about the causes and consequences of the threat for which healthy/precautionary behavior is recommended

13. Past buhavior

14. Perceived barriers to behaving in a healthy/ precautionary way

15. Perceived benefits of healthy/precautionary behavior

16. Risk perceptions for the condition for which healthy/precautionary behavior is recommended

17. Self esteem to change unhealthy /risky behavior

18. Social pressure to change unhealthy/risky behavior

19. The "Consciousness-raising" process of change

20. The "Dramatic relief" process of change

21. The "Envirommental re-evaluation" process of change

$\begin{array}{llllll}75 & 2 & 2 & 48 & 2 & 0 \\ 75 & 2 & 1 & - & - & \end{array}$

$\begin{array}{llllll}75 & 2 & 2 & 48 & 2 & 1.50 \\ 74 & 1 & 2 & 48 & 1 & 1\end{array}$

$\begin{array}{llllll}71 & 1 & 2 & 48 & 1 & 1.75\end{array}$

$\begin{array}{llllll}72 & 0 & 3 & 47 & -1 & 3\end{array}$

$\begin{array}{llllll}71 & 1 & 3 & 45 & 1 & 1\end{array}$

$\begin{array}{llllll}71 & 1 & 2 & 46 & 1 & 2\end{array}$

$\begin{array}{llllll}71 & 1 & 2 & 46 & 1 & 2\end{array}$

$\begin{array}{llllll}73 & 2 & 2 & 48 & 2 & 0.75\end{array}$

$\begin{array}{llllll}74 & 1 & 3 & 48 & 1 & 1.75\end{array}$

$\begin{array}{llllll}65 & 0 & 2 & 48 & 0 & 2\end{array}$

$\begin{array}{llllll}72 & 1 & 3 & 48 & 1 & 1.75\end{array}$

$\begin{array}{llllll}73 & 1 & 2 & 48 & 1 & 2\end{array}$

$\begin{array}{llllll}71 & 1 & 2 & 48 & 1 & 1.75\end{array}$

$\begin{array}{llllll}65 & 0 & 2.5 & 48 & 0 & 2\end{array}$

$\begin{array}{llllll}73 & 1 & 3 & 48 & 1 & 1\end{array}$

$\begin{array}{llllll}71 & 2 & 2 & 46 & 2 & 1\end{array}$

$\begin{array}{llllll}63 & 1 & 3 & 46 & 1 & 2\end{array}$

$\begin{array}{llllll}66 & 1 & 2.25 & 45 & 1 & 1.50\end{array}$




\begin{tabular}{ll}
\hline & Second round \\
Item & $\mathrm{N}$ Third round \\
\hline
\end{tabular}

\section{B. Transition from the contemplation stage to preparation stage}

1. Actual skills to behave in a healthy/ precautionary way

2. Anticipated regret about not behaving in a healthy/precautionary way

3. Awareness of the personal relevance to change unhealthy/risky behavior

4. Awareness of the relation between unhealthy/risky behavior and health problems

5. Awareness that you are behaving in a unhealthy/risky way

6. Beliefs about the effectiveness of healthy/precautionary behavior in preventing health problems

7. Cognitive attitudes towards healthy/precautionary behavior

8. Competence of the person that has to change unhealthy/risky behavior

9. Cues to action to behave in a healthy/precautionary behavior

10. Emotions involved with unhealthy/risky behavior

11. Emotions involved with healthy/precautionary behavior

12. Experiencing a symptom that might be related to behaving in an unhealthy/risky way

13. Experience with people with a symptom or condition for which the healthy/ precautionary behavior is recommended

14. Implementation intentions with regard to behaving in a healthy/precautionary way

15. Knowledge about the recommendations related to healthy/precautionary behavior

16. Offer of help from others to change unhealthy/risky behavior

17. Perceived barriers to behaving in a healthy/precautionary way

18. Perceived, benefits of healthy/precautionary behavior

\begin{tabular}{|c|c|c|c|c|c|}
\hline 69 & -1 & 3 & 47 & -1 & 2 \\
\hline 75 & 2 & 2 & 48 & 2 & 0 \\
\hline 75 & 2 & 1 & - & - & $\therefore$ \\
\hline 75 & 2 & 2 & 48 & 2 & 1.50 \\
\hline 74 & 1 & 2 & 48 & 1 & 1 \\
\hline 71 & 1 & 2 & 48 & 1 & 1.75 \\
\hline 72 & 0 & 3 & 47 & -1 & 3 \\
\hline 71 & 1 & 3 & 45 & 1 & 1 \\
\hline 71 & 1 & 2 & 46 & 1 & 2 \\
\hline 71 & 1 & 2 & 46 & 1 & 2 \\
\hline 73 & 2 & 2 & 48 & 2 & 0.75 \\
\hline 74 & 1 & 3 & 48 & 1 & 1.75 \\
\hline 65 & 0 & 2 & 48 & 0 & 2 \\
\hline 72 & 1 & 3 & 48 & 1 & 1.75 \\
\hline 73 & 1 & 2 & 48 & 1 & 2 \\
\hline 71 & 1 & 2 & 48 & 1 & 1.75 \\
\hline 65 & 0 & 2.5 & 48 & 0 & 2 \\
\hline 73 & 1 & 3 & 48 & 1 & 1 \\
\hline
\end{tabular}


Table 1.1 - continued

Results of the Delphi study per item (second and third round)

$\begin{array}{lll} & \text { Second round } & \text { Third round } \\ \text { item } & \text { N Mdn IQD N Mdn IQD }\end{array}$

B. Transition from the contemplation stage to preparation stage

19. Risk perceptions related to the condition for $\quad \begin{array}{lllllll}71 & 2 & 2 & 46 & 2 & 1\end{array}$ which healthy/precautionary behavior is recommended

20. Self-efficacy to behave in a

$\begin{array}{llllll}63 & 1 & 3 & 46 & 1 & 2\end{array}$
healthy/precautionary way

21. Self esteem to change unhealthy/risky behavior

22. The "Dramatic relief" process of change

$\begin{array}{llllll}66 & 1 & 2.25 & 45 & 1 & 1.50\end{array}$

23. The "Environmental re-evaluation" process of $\begin{array}{llllll}64 & 1 & 2 & 46 & 1 & 1\end{array}$

$\begin{array}{llllll}66 & 1 & 2 & 45 & 1 & 1\end{array}$
change

24. The "Self re-evaluation" process of change

$\begin{array}{llllll}66 & 2 & 2 & 45 & 1 & 1\end{array}$

25. Subjective norms about healtixy/precautionary

$\begin{array}{llllll}68 & 1 & 2 & 47 & 1 & 1\end{array}$
behavior

\section{c. Transition from the preparation stage to the action stage}

1. Actual skills to behave in a healhy/ precautionary 7 way

2. Anticipated regret about not behaving in a 69 healthy/ precautionary

3. Commitment to behave in a healthyl precautionary way

4. Cues to action to behave in a healthy/ precautionary way

5. Implementation intentions with regard to behaving $\begin{array}{llll}71 & 2 & 1\end{array}$ iw a healthy/precaulionary way

6. Overcoming practical barriers to behaving in a

$\begin{array}{lll}72 & 3 & 1\end{array}$
healthy/precationary way

7. Perceived social reactions to intending to change unhealthy/ risky behavior

8. Risk perceptions related to the condition for which the healthy/precautionary behavior is

1

248

48 reconmended

9. Self-eficacy to behove in a healthy/ precautionary way

10. Social support to behave in a healthy/ precautionary way

$\begin{array}{llllll}71 & 1 & 2 & 46 & 1 & 1.25 \\ 70 & 0.50 & 2.25 & 47 & 0 & 1 \\ 72 & 3 & 1 & - & - & - \\ 72 & 2 & 1.75 & 47 & 2 & 1\end{array}$


Item

Second round

Third round

D. Transition from the action stage to maintenance stage

1. Anticipated regret about (re)lapse to

N Mdn IQD N Mdn IQD unhealthy/risky behavior

2. Coping skills to resist social pressure to behave in an whealthy/risky way

3. Coping skills to resist temptations to revert to unhealthy/risky behavior

4. Difficulty of behaving in a healthy/ precautionary way

5. Extent to which behaving in a healthyl precautionary way has become habitual

6. Feedback about success of behaving in a healthy/precautionary way

7. Feedback from respected others about behaving in a healthy/precautionary way

8. Implementation intentions with regard to beharving in a healthy/precautionary way

9. Insight into different aspects of changing behavior in generall

10. Perceived social reactions to behaving in a healthy/precautionary way

11. Physiological reactions to behaving in a healthy/precautionary way

12. Psychological reactions to behaving in a healthy/precautionary behavior

13. Re-evaluation of expected pros and cons of healthy/precautionary behavior

14. Self-efficacy to maintain healthy/precautionary behavior

15. Skills to cope with difficult situations that may hinder healthy/ precautionary behavior

16. Social support to maintain healthy/precautionary $\begin{array}{lll}72 & 2\end{array}$ behavior

17. Will-power

$\begin{array}{llllll}64 & 1 & 3.75 & 47 & 0 & 2\end{array}$

Note. All items of the questionnaires are included in this table. Dashes indicate that consensus was obtained on the item in the second round and was excluded from the third round questionnaire.

${ }^{a}$ All items showed a range of -3 to 3 , except item AI (range: $-3,1$ ) and A13 (range: $-3,2$ )

- $M d n=$ Median scores, $I Q D=$ Interquartile dewiations

* Items on which consensus was obtained in the second round are prirted in italics 
Largest consensus among respondents was obtained about determinants of the latter stage transitions. For the preparation to action transition, consensus was obtained on 5 out of 10 items. Least consensus was achieved for the precontemplation to contemplation transition, where only 1 out of 21 items resulted in an IQD $\leq 1$ (see Table 1.2).

Table 1.2 Percentage and number of items on which agreement was reached presented by stage transition

\begin{tabular}{llllll} 
& \multicolumn{3}{c}{ Percentage of items on which agreement was reached } \\
\cline { 2 - 6 } & \multicolumn{3}{c}{ Second round } & \multicolumn{3}{c}{ Third round } \\
\cline { 2 - 6 } Stage transition & $\%$ & $\mathrm{~N}$ & $\%$ & $\mathrm{~N}$ \\
Precontemplation to Contemplation & 4.8 & 1 & 30 & 6 \\
Contemplation to Preparation & 24 & 6 & 63.2 & 12 \\
Preparation to Action & 50 & 5 & 60 & 3 \\
Action to Maintenance & 35.3 & 6 & 72.7 & 8 \\
\hline
\end{tabular}

\section{Third round}

\section{Consensus and agreement with stage transition determinants}

In the third round, consensus was achieved for 29 items (IQD $\leq 1)$. None of these items revealed a median score of three (fully agree with the item, Table 1.1). Thirteen items had a median score of two, representing the "strongly agree with the item" opinion and another 13 items had a median score of one, representing the "slightly agree with the item" option. Respondents strongly agreed that the transition from precontemplation to contemplation is determined by awarenessrelated factors such as awareness of the personal relevance of changing unhealthy/risky behavior, experiencing a symptom that might be related to unhealthy/risky behavior, and the environmental re-evaluation process of change.

Respondents also strongly agreed that implementation intentions, offer of help from others, self-efficacy, and self-esteem could be determinants of the transition from contemplation to preparation. Social support was considered to determine transition from preparation to action.

The action-maintenance transition was perceived to be determined by feedback-related issues like feedback about success, feedback from respected others, physiological reactions and psychological reactions to behaving in a healthy/precautionary way, and the difficulty of behaving in a healthy/precautionary way. 
For the precontemplation to contemplation transition, consensus was obtained on 6 out of 20 items. For the contemplation to preparation transition, consensus was obtained on 12 out of 19 items. For the transitions from preparation to action stage and from action to maintenance, consensus was obtained for 3 out of 5 and 8 out of 11 items, respectively (Table 1.2). Table 1.3 provides a summary of items on which consensus was obtained for each of the stage transitions.

\section{Table 1.3 Items on which consensus was obtained per stage transition}

\begin{tabular}{ll}
\hline Stage transition & Transition determinants \\
\hline PC-C & Awareness of personal relevance to change \\
& Awareness of relation between behavior and health problems \\
& Experiencing a symptom that might be related to unhealthy \\
behavior & Consciousness raising process of change \\
Awareness of personal relevance to change & Experiencing a symptom that might be related to unhealthy \\
behavior & Implementation intentions \\
& Offer of help from others \\
& Pexceived benefits \\
& Self-efficacy \\
& Self-esteem \\
Actual skills & Commitment to act \\
Implementation intentions & Overcoming practical barriers \\
& Self-efficacy \\
Social support & Coping skills to resist social pressure \\
Coping skills to resist temptations \\
Difficulty of behaving healthy \\
Extent to which behavior has become habitual \\
Feedback about success \\
Psychological reactions to behaving healthy \\
Self-efficacy \\
Skills to cope with difficult situations \\
Social support \\
A - M
\end{tabular}

Note. Only items on which participants collectively strongly agreed (Mdn $\geq 2$ ) that they influence forward stage transition are depicted. $P C=$ precontemplation, $C=$ contemplation, $P R=$ preparation, $A$ $=$ action, $\mathrm{M}=$ maintenance 


\section{Changes in consensus}

In the third round significant changes in consensus were found for all stage transitions. For determinants of the transition from precontemplation to contemplation $(z=-3,42 ; p=.001)$, from contemplation to preparation $(z=-3.62 ; p$ $=.001$ ), from preparation to action $(z=-2.03 ; p=.042)$, and from action to maintenance $(z=-2.83 ; p=.005)$ larger consensus was found in the third round as compared to the second round.

\section{Discussion}

The present study aimed to make a systematic inventory of opinions of experts on determinants of forward transitions in Transtheoretical stages of change. The results showed that the respondents agreed on various stage transition determinants, but also that these determinants were not always stage-specific. In sum, awareness-related issues were considered to be important in the early stage transitions between precontemplation and preparation. The concept of implementation intentions was identified as an important determinant for transitions between contemplation and action. Self-efficacy and (perceived) control-related issues, as well as social support were identified as important determinants of all transitions from contemplation to maintenance. Consensus was obtained on only few stage-specific determinants: decisional balance was considered important for the contemplation-preparation transition, commitment to act for preparation to action, and different feedback possibilities about the (effects of the) behavior change for action to maintenance. The results will be discussed per stage transition and compared to previous studies. This comparison will be made with prospective studies, since such studies are better suited to identify predictors of stage transitions then cross-sectional studies.

The role of awareness in the precontemplation-contemplation stage transition has been proposed and studied before. For example, De Bourdeaudhuij and Brug (2000) have shown in a randomized trial, that making people aware of their personal intake levels of dietary fat did result in stronger intentions. The role of the consciousness raising process, which is similar to awareness, has also been studied longitudinally but no evidence for transition prediction was found (Herzog, Abrams, Emmons, Linnan, \& Shadel, 1999).

According to respondents, issues related to awareness and control, implementation intentions, social support and decisional balance might determine the transition from contemplation to preparation. No studies are 
known on the role of implementation intentions and self-esteem in stage transitions. A few prospective studies supported the idea that social support might play a role in the transition from contemplation to preparation (Courneya et al., 2001; De Vries \& Mudde, 1998). Further, some prospective studies have been conducted to investigate the role of decisional balance in early stage transitions in smoking cessation with inconsistent results. Velicer et al: (1999) found that measures of pros and cons effectively detect changes as individuals move between stages of change. Etter \& Perneger (1999) only found support for the cons, whereas others (De Vet, De Nooijer, De Vries, \& Brug, 2005a; Dijkstra, Tromp, \& Conijn, 2003) did not find any support for pros and cons as predictors of stage transition from contemplation to preparation. Self-efficacy or perceived behavioral control has been found to be related to progressing from contemplation (Courneya et al., 2001), although other studies identified self-efficacy to be most important for progress from preparation (De Vries \& Mudde, 1998; Dijkstra et al., 2003).

Once someone is in preparation, initiating behavior change seems, according to the respondents in the present study, to be related to skills, implementation intentions, overcoming practical barriers, self-efficacy, social support and commitment to act. As far as we know, to date no formal stages of change related prospective analyses have been done on skills, implementation intentions and practical barriers as potential transition determinants. Nevertheless, a number of studies have pointed out that implementation intentions can be effective in bridging the intention-behavior gap (Gollwitzer, 1999; Sheeran, 2002). Segan and colleagues (2002) have found support for commitment to act and self-efficacy as predictors of the transition from preparation to action. Courneya et al. (2001) examined the role of social support, but did not find this concept predictive of progression from preparation.

Control and feedback-related issues can be related to maintaining the initiated behavior change according to the respondents. In the area of addictive behaviors a focus thas been on strategies to maintain behavior change, so-called relapse prevention. One of the central ideas in relapse prevention is that addictive habits can be changed through the application of self-control procedures (Marlatt \& Gordon, 1985). Not much is known from empirical literature about relapse prevention in relation to the action-maintenance transition. Most prospective studies done on stage transitions either do not make a distinction between action and maintenance stages (Courneya et al., 2001; Plotnikoff et al., 2001; Velicer et al., 1999) or focus on the pre-action stage transitions only (De Vries \& Mudde, 1998; Etter \& Perneger, 1999; Herzog et al., 1999; Segan et al., 2002). Dijkstra et al. (2003) specifically examined the transition from action to maintenance and found indications that lower scores on pros of smoking and higher scores on selfefficacy were relevant factors for forward stage movement for people in smoking 
cessation action. Armitage and colleagues (2004) also found higher scores on selfefficacy to be predictive of progress from action in dietary fat reduction.

A notable finding of the present study is that consensus about earlier stage transitions falls far behind consensus about determinants of later stage transitions. In the first round, more possible determinants were identified for the early stage transitions (e.g., precontemplation to contemplation) than for the later stage transitions. Also, in the second and third round, lower IQDs and higher median scores were found for determinants of later transitions from preparation to action and from action to maintenance, showing larger consensus on determinants of these stage transitions. These results may reflect the strong emphasis in health education and promotion programs on getting people to action. Before stages of change models came into fashion, behavior change has implicitly been regarded as a two-stage process, from unhealthy to healthy behavior. Most experience may therefore be with action-initiating interventions. The fact that more potential determinants of the earlier stage transitions were proposed might also indicate that these stage transitions are more complex, including more potential stage transition determinants, dependent on the behavior under study.

Some limitations of this Delphi study must be discussed. First, the respondents may not be representative for 'all' experts on stages of change, due to non-optimal response rates. However, when using mail questionnaires a response rate of $38 \%$ is not exceptional (Swanborn, 1987). Lack of time was the main reason reported for not participating in the Delphi study, indicating that non-response was not related to attitude towards stages of change. Furthermore, the study population (except for the first round) consisted only of authors of papers on stages of change. This may have led to biased results. However, the respondents represented authors of papers very critical as well as very supportive toward stage of change. An additional limitation lies within the group process. Since participants were not offered the opportunity to justify their opinions by use of argumentation, all participants" opinions were regarded as equally important. Besides, most authors conducted only a single study on TTM, while others have conducted several studies. Possibly, some participants were more experienced with TTM than others.

Since the time investment that can be asked from participants is limited, only forward stage transition determinants were studied, although we acknowledge that backward transition determinants are also interesting for behavior change and these might differ from forward transition determinants. However, as noted in the introduction, from a planned health promotion perspective an integration of forward stage transition determinants would be more interesting, since motivating people to adopt and maintain healthier lifestyles is one of the central goals in the field. 
To conclude, the present study revealed some integrative insights into stage transition determinants from a multiple-theory perspective, based on knowledge and experiences from experts in the field. It would have been complex to derive these insights from the literature, because most empirical studies have only included few possible stage change determinants and some potential stage change determinants have not been studied in relation to stages of change yet (e.g., implementation intentions). The Delphi study approach thus provided the opportunity to identify a broader spectrum of potential stage transition determinants. A next step would be to conduct experimental and longitudinal studies to test if these determinants do predict forward stage transitions. 


\title{
Chapter 2 \\ Determinants of forward stage transition from precontemplation and contemplation for fruit consumption
}

\begin{abstract}
The present study aimed to gain insight into cross-sectional and longitudinal associations between decisional balance, self-efficacy, fruit intake, and stage of change transition out of precontemplation and contemplation. A longitudinal cohort study with 735 individuals was conducted, using electronic questionnaires. Of the cohort, 648 (response rate $=88 \%$ ), 592 (response rate $=$ $81 \%$ ), and 570 (response rate $=78 \%$ ) respondents completed questionnaires at $T 1$, 53 days after T1 (T2), and 106 days after T1 (T3), respectively. The two equal time intervals of 53 days were analyzed longitudinally and three points in time cross-sectionally. Logistic regression analyses showed that pros, fruit intake and self-efficacy predicted forward stage transition from precontemplation. Selfefficacy predicted forward stage transition from contemplation. Cons did not predict stage transitions. Results from longitudinal analyses were similar to cross-sectional results, except for self-efficacy: no differences between early stages in self-efficacy were found, while self-efficacy predicted these early stage transitions. To conclude, within the limitations posed by the sampling frame, results provided support for the Transtheoretical Model, although determinants might not always be stage-specific.
\end{abstract}

Published as: De Vet, E., De Nooijer, J., De Vries, N. K., \& Brug, J. (2005). Determinants of forward stage transition from precontemplation and contemplation for fruit consumption. American Journal of Health Promotion, 19, 278-285. 


\section{Introduction}

The Transtheoretical Model (TTM) provides a framework for research on the process of health behavior change and for developing and evaluating health promoting interventions (Prochaska et al, 1992; Prochaska \& Velicer, 1997a). The TTM postulates that behavior change is a dynamic process, which involves movement through a sequence of discrete, qualitatively distinct stages. The core organizing construct of the TTM is the Stages of Change construct, which represents a temporal dimension and distinguishes five stages of change: precontemplation (no intention to change behavior within the next six months), contemplation (intention to change behavior within the next six months), preparation (intention to change behavior within 30 days), action (people changed from unhealthy to healthy behavior within the past six months), and maintenance (maintenance of the behavior change for more than six months; Prochaska et al., 1992; Prochaska \& Velicer, 1997a). People in these different stages are assumed to differ on important and relevant factors (Weinstein et al., 1998), such as self-efficacy, and the pros and cons of adopting the behavior. If such factors predict movement to a next stage in the sequence (stage transition), they are known as stage transition determinants. According to the TTM, people in different stages of change benefit most from interventions matched to the relevant stage transition determinants to induce stage transition. Matching interventions to stage of change is called stage-matching or stage-tailoring (Prochaska \& Velicer, 1997a; Weinstein et al., 1998). Insight into stage transition determinants is needed in order to develop effective stage-matched interventions.

To date, most research on stage theories of health behavior has been cross-sectional. In such studies people are classified into stages of change and compared on potential stage transition determinants. Differences between the stages of change on such variables may indicate that these variables induce stage transition. As Sutton (2000b) pointed out, problems exist in interpreting these cross-sectional data. More specifically, when a theoretical variable differs between stages of change, there are no indications whether the theoretical variable influences stage transition or that stage transition influences this theoretical variable or that a third variable influences both the theoretical variable and stage transition (Sutton, 2000b). Longitudinal analyses can provide more insight into potential determinants of stage transitions. The present study, therefore, aimed to replicate results from cross-sectional analyses of differences in potential determinants between stages of change with results based on longitudinal analyses. 
In the present study, we measured stages of change related to fruit intake since ample consumption of fruit is considered to contribute to the prevention of cardiovascular disease (Trichopoulou \& Lagiou, 1997), certain cancers (Key, Allen, Spencer, \& Travis, 2002; Paolini, Sapone, Canistro, Antonelli, \& Chieco, 2003), as well as obesity (Lanza et al., 2001; Pesa \& Tumer, 2001); while fruit intake is below recommended intake levels in many countries, including the US and most countries in Europe (Dutch Nutrition Centre, 1998; Trudeau, Kristal, Li, \& Patterson, 1998). In the Netherlands, approximately $70 \%$ of the population eat less than the recommended minimum amount of two servings (250 grams) of fruit per day (Dutch Nutrition Centre, 1998; Van Oers, 2002) Furthermore, fruit intake has previously been related to stage of change in cross-sectional studies (Brug et al., 1997; Campbell et al., 1998, 1999; Horacek et all, 2002; Ling \& Horwath, 1999; Ma et al., 2002; Van Duyn et al., 2001; Van Duyn et al., 1998).

Decisional balance (i.e., pros and cons) and self-efficacy have been proposed as potential stage transition determinants, and research using crosssectional methods has provided some support for this proposition (Brug et al., 1997; Campbell et al., 1998; Ling \& Horwath, 1999; Van Duyn et al., 2001). Decisional balance refers to the individual's weighing of pros and cons of a behavior and is usually measured by rating the importance of possible perceived pros and cons of the behavior (Prochaska et al., 1994; Velicer, DiClemente, Prochaska, \& Brandenburg, 1985). According to the TTM, the pros must increase to progress from precontemplation to contemplation (Prochaska, 1994). This was confirmed by $\mathrm{Ma}$ and colleagues (2002) and also by Horacek and colleagues (2002), but only for the males in their sample. Van Duyn et al. (2001) found lower pros scores in pre-action than in action, and in action than in maintenance, but did not examine precontemplation, contemplation, and preparation separately. Campbell et al. (1998) did not find significant differences between stages in pros of changing.

According to the TTM, the cons must decrease between contemplation and action (Prochaska, 1994). This prediction was only partially supported by cross-sectional data. Van Duyn et al. (2001) found higher cons scores in preaction stages than in the action stage, whereas Ma et al. (2002) found higher cons scores in contemplation than in maintenance. Further, differences in cons of fruit intake were also found between precontemplation and contemplation. Ma et all. (2001) found higher cons scores in contemplation than in precontemplation, while Campbell et al. (1998) showed the opposite result. Horacek et al. (2002) did not find significant results for the cons of fruit intake.

In the TTM, self-efficacy refers to the situation-specific confidence people have that they can cope with high-risk situations without relapsing to their unhealthy or high-risk habit (Prochaska \& Velicer, 1997a). Self-efficacy can 
be represented by a monotonically increasing function across the five stages (Plummer et al., 2001; Velicer et al., 1998). Plummer et all. (2001) postulated that the self-efficacy measures are particularly sensitive to changes that are involved in progress in the later stages of change and lack of self-efficacy may be a good predictor of relapse. Most cross-sectional analyses confirmed this idea, showing higher self-efficacy scores in action than in pre-action stages (Brug et al., 1997; Horacek et al, 2002; Ling \& Horwath, 1999; Ma et al., 2002; Van Duyn et al., 2001). Further $r_{r}$ several studies also found self-efficacy to differ between the precontemplation and the contemplation/preparation stage. In general, selfefficacy scores were higher in contemplation/preparation than in precontemplation (Brug et al., 1997; Horacek et al., 2002; Ma et al., 2002), although Ling and Horwath (1999) showed the opposite result.

To date, these associations between theoretical determinants and stages of change in fruit intake have not been studied using a longitudinal design. In the present study we examined: (1) whether pros, cons, self-efficacy, and fruit intake differ between stages of change at three points in time (cross-sectional analyses) as suggested by the TTM, and (2) whether pros, cons, self-efficacy and fruit intake predict forward stage transition out of precontemplation and contemplation for two time periods (longitudinal analyses). For the TTM variables we examined whether these variables separately predict stage transitions and whether the complete TTM predict stage transitions.

We focus on forward stage transition out of precontemplation and contemplation, since most respondents were in these stages and it narrows the scope of this paper in order to provide a more in-depth analysis of possible determinants of stage transitions.

\section{Method}

\section{Design}

A cohort of 735 respondents was emailed the web address of the electronic questionnaire at the start of the present study (T1), 53 days later (T2) and again after another 53 days (T3). Respondents also received a personal login code to open and complete the questionnaires and were asked to complete the questionnaire in a single session within one week after receiving the web address by email. Respondents could not save their answers or complete the questionnaire more than once. Respondents' answers were automatically entered into a data file, and after the one-week deadline had passed by, the respondents could not enter the questionnaire anymore. A baseline (TO) 
questionnaire was used to invite potential respondents and to describe the cohort on demographic characteristics and fruit intake, and to classify respondents into stages of change. For $\mathrm{T} 1, \mathrm{~T} 2$ and $\mathrm{T} 3$ the electronic questionnaires were identical and included items measuring stage of change, pros, cons and self-efficacy, and a food frequency questionnaire to assess fruit intake.

The data were analyzed cross-sectionally for the three measurements separately ( $\mathrm{T} 1, \mathrm{~N}=648$, response rate $88 \%$; $\mathrm{T} 2, \mathrm{~N}=592$, response rate $81 \%$; $\mathrm{T} 3$, $\mathrm{N}=570$, response rate $78 \%$ ), and longitudinally for the two equal time intervals of 53 days between these three measurements, $\mathrm{T} 1-\mathrm{T} 2(\mathrm{~N}=552$, response rate $75 \%)$ and $\mathrm{T} 2-\mathrm{T} 3(\mathrm{~N}=518$, response rate $70 \%)$, to compare differences between stages of change and predictors of stage transitions. Three measurements and two time intervals were analyzed in order to try to replicate the results within the present study. Further, test-retest reliability for all the relevant variables was assessed at the start of the study, using two measurements with a 14-day time interval.

\section{Sample}

Participants were identified from a Dutch Internet panel sized 8,165 persons at the time of study. Individuals indicated their willingness to become a panel member on the website of Flycatcher Internet Research BV (www.flycatcher.nl). The total panel consisted of $43 \%$ males and $57 \%$ females, and the mean age was 33 years. Of the panel members, $23 \%, 37 \%$ and $40 \%$ had a low, medium or high level of education, respectively. From this Internet panel, a random sample of 1,500 people aged 18 years and older was invited by an email letter to participate in a larger longitudinal cohort study. Respondents could indicate their willingness to participate in the entire study by completing an electronic questionnaire used to identify respondents (TO). Completed questionnaires were returned by 929 respondents (response rate $=62 \%$ ). Thirteen respondents were excluded from the study because stage of change or fruit intake could not be calculated $(n=916$, response rate $=61 \%)$. Of the remaining 916 respondents, $20 \%$ of the respondents in each of the baseline stages of change, were selected randomly to participate in a different study that will be reported elsewhere. The remaining 735 respondents participated in the present study.

In total, $488(66 \%)$ respondents completed the three questionnaires. Respondents who did not complete all three questionnaires were significantly younger $(F(1,735)=25.90, p<.001)$ and are more often female $\left(\chi^{2}(1, N=735)=\right.$ $5.00 ; p<.05)$ than those who completed all three questionnaires. No differences between completers and non-completers were found with respect to level of education, ethnicity, stage of change or fruit intake. 


\section{Measures}

Stage of change was operationalized by a single-item algorithm consistent with Armitage and Arden (2002) and Laforge et al. (1999) and adapted to fruit intake by asking respondents to select from five statements, each representing a stage of change, the most applicable to their personal situation: "I do not eat at least two servings of fruit every day and I do not intend to change this within the next six months" [precontemplation], "I do not eat at least two servings of fruit every day, but I intend to change this within the next six months" [contemplation], "I do not eat at least two servings of fruit every day, but I intend to change this within the next month" [preparation], "I eat at least two servings of fruit every day, and I have started doing this in the last six months" [action], "I eat at least two servings of fruit every day, and I have done so for more than six months" [maintenance]. Test-retest reliability with a two-week interval showed a Kappa .57, $p<.001$ for stages of change.

Decisional balance for fruit intake was assessed with 16 items based on the decisional balance scale for fruit and vegetable intake developed by $\mathrm{Ma}$ et al. (2002). Pros (other people eat fruit; fruit can substitute for unhealthy foods; fruit is good for your body; a diet is more varied when you eat fruit; eating fruit can help to prevent diseases; eating fruit makes you feel better; eating fruit can help to prevent weight gain; eating fruit can help you lose weight) and cons (eating fruit is expensive; fruit spoils quickly; fruit recommendations are unclear; it is inconvenient to eat fruit; it is difficult to find tasty fruit; it is difficult to keep fruit; it takes time to buy fruit; worry about chemicals on fruit) were assessed by asking respondents how important each item was in their decision making for eating at least two servings of fruit each day on a 5-point Likert scale ranging from -2 (not at all important) to 2 (very important). For example, "How important are the following factors in your decision making to eat at least two servings of fruit each day? Eating fruit is expensive." Cronbach's $\alpha$ were .75 and .74 for pros and cons respectively, which were comparable to the Cronbach's $\alpha$ found by Ma et al. (2002; .73 and .72 for pros and cons respectively). A mean score was computed for pros and cons separately. Test-retest reliability with a two-week interval showed a Pearson correlation of $r=.70, p<.001$ for the pros, and $r=.71$, $p<.001$ for the cons.

Self-efficacy was assessed by asking respondents to rate how difficult or easy eating at least two servings of fruit each day was on a 5-point Likert scale ranging from -2 (very difficult) to 2 (very easy) for six high-risk situations: during weekends, during working days, in winter, when you don't have much time, when you experience (emotional) distress, and when having a touch of flu or a cold, which were identified based on qualitative research. For example, "How difficult or easy do you think it is to eat at least two servings of fruit each day during weekends?" Principal Component Analyses extracted one component, 
with factor loadings ranging from .64 to .87 . A mean score for self-efficacy was computed (Cronbach's $\alpha=.88$; test-retest reliability $r=.83, p<.001$ ).

Fruit intake was assessed with a 14-item food frequency questionnaire (FFQ) that was validated using a 7-day dietary record $\left(r=.51_{i} p<.001\right.$; Van Assema, Brug ${ }_{i}$ Ronda, Steenhuis, \& Oenema, 2002) and biomarkers for fruit consumption levels, i.e., blood levels of total carotenoids $(r=.34 ; p<.001)$, vitamin $C(r=.27 ; p<.001)$, and beta-cryptoxanthin $(r=.42 ; p<.001$; Bogers, Van Assema, Kester, Westerterp, \& Dagnelie, 2004). Respondents were asked about consumption during the previous month. The FFQ included questions about frequency of consumption (from "never/less than one time per month" to " 7 days a week") and number of servings (pieces or small bowls [for small fruit such as berries]) per day that a certain fruit (juice) was consumed. Separate questions were included for the most common fruits in the Netherlands (citrus fruit, such as oranges and grapefruit; apples and pears; bananas), for an important seasonal fruit (tangerines), for freshly squeezed or other unsweetened fruit juice, and for "other fruit including preserved fruit". To calculate total fruit consumption, serving sizes were first transformed to grams based on standard mean weights of the fruits. Subsequently, for each fruit category frequency of consumption was multiplied with serving size and the results were added up and divided by seven to obtain a fruit intake score in grams per day. Test-retest reliability in the present study was $r=.68, p<.001$.

Demographic variables included age, sex, education, and ethnicity, and were only assessed at T0. Education was measured by asking the respondents about the highest level of education they had completed. Respondents who indicated that they had no education, primary school, secondary school, lowest level of high school, or lower vocational training were classified as having a low level of education. Respondents with a medium level of education had completed intermediate or high level high school, or medium level vocational training. Respondents who had completed higher vocational training, college, or university education had a high level of education. Ethnicity of respondents was measured by asking respondents the country of birth for both parents. If one or both parents had been born outside the Netherlands, respondents were classified as "of foreign origin". In case both parents had been born in the Netherlands, respondents were classified as "of Dutch origin".

\section{Analyses}

Descriptive statistics were used to describe the study population at baseline and stage transitions out of precontemplation and contemplation for both time intervals. ANOVA with Scheffé multiple-comparison test (alpha level $=.05$ ) was used to detect differences in age between baseline stages of change. Chi-square 
analyses were used to detect differences in sex, level of education, and ethnicity between baseline stages of change.

To examine differences in pros, cons, self-efficacy, and fruit intake between stages of change at $\mathrm{T} 1, \mathrm{~T} 2$, and $\mathrm{T} 3$ cross-sectional analyses were conducted using ANOVA with Scheffé multiple-comparison test (alpha level = .05).

Since the sample size was not sufficient to examine all possible patterns of stage change, for both time intervals stage transitions were transferred into a dichotomous variable for precontemplation $(1=$ precontemplators who progressed at least one stage; $0=$ precontemplators who remained stable) and contemplation $(1=$ contemplators who progressed at least one stage; $0=$ contemplators who remained stable or regressed).

To explore and test predictors of forward stage transition out of precontemplation and contemplation, logistic regression analyses were conducted with forward stage transition between $\mathrm{T} 1$ and $\mathrm{T} 2$ as dependent variable and pros, cons, self-efficacy, and fruit intake at $\mathrm{T} 1$ as independent variables. First univariate analyses were conducted for each potential predictor. Subsequently, all potential predictors from the TTM were tested in one multivariate model using forced entry. The exact same analyses were conducted for $\mathrm{T} 2-\mathrm{T} 3$ to replicate the results found for the T1-T2 interval.

\section{Results}

\section{Participants}

The mean age of participants was $37.5(\mathrm{SD}=14.1)$ years, and women and men were equally represented ( $51 \%$ vs. $49 \%$ ). Of the respondents, $17 \%, 48 \%$, and $36 \%$ reported a low, medium and high level of education, respectively. Most respondents were of Dutch origin (90\%). Mean fruit intake was 260 grams per day $(\mathrm{SD}=163)$, while $57 \%$ of the respondents ate less fruit than the recommended minimum intake level of 250 grams a day. At baseline (T0), 242 $(33 \%)$ respondents were in precontemplation, $157(21 \%)$ in contemplation, 104 $(14 \%)$ in preparation, $49(7 \%)$ in action, and $183(25 \%)$ in maintenance. At baseline, significant differences in age and sex were found between stages of change with (1) a higher mean age in maintenance than in all other stages ( $F$ ( 4 , $735)=12.12 ; p<.001$ ) and (2) more males and fewer females in precontemplation and maintenance, and more females and fewer males in contemplation, preparation and action $(\chi 2(4, N=735)=14.83 ; p<.01)$. No significant differences between baseline stages of change were found for level of education and ethricity. 


\section{Stage transitions}

Table 2.1 presents stage transitions out of precontermplation and contemplation for the two time intervals. Of the respondents in precontemplation at $T 1,39$ (23\%) progressed to contemplation, preparation, action or maintenance between $\mathrm{T} 1$ and $\mathrm{T} 2$. Of the respondents in precontemplation at $\mathrm{T} 2,27(17 \%)$ progressed to a later stage between $\mathrm{T} 2$ and $\mathrm{T} 3$. Of the respondents in contemplation at $\mathrm{T} 1,41$ $(35 \%)$ respondents progressed out of contemplation to preparation, action or maintenance between $\mathrm{T} 1$ and $\mathrm{T} 2$. Of the respondents in contemplation at $\mathrm{T} 2,26$ ( $24 \%$ ) progressed between $\mathrm{T} 2$ and $\mathrm{T} 3$.

\begin{tabular}{|c|c|c|c|c|c|}
\hline \multicolumn{6}{|c|}{ Posttest } \\
\hline Pretest & $P C^{a, b}$ & c & PR & $\mathbf{A}$ & $M$ \\
\hline \multicolumn{6}{|c|}{$\mathrm{T}_{2}$} \\
\hline \multicolumn{6}{|c|}{ T1 (\% within T1 $)^{c}$} \\
\hline PC & $131(77.1 \%)$ & $23(13.5 \%)^{4}$ & $5(2.9 \%)$ & $4(2.4 \%)$ & $7(4.1 \%)$ \\
\hline$c$ & $16(13.8 \%)$ & $59(50.9 \%)$ & $20(17.2 \%)$ & $18(15.5 \%)$ & $3(2.6 \%)$ \\
\hline \multicolumn{6}{|c|}{$\mathrm{T}_{3}$} \\
\hline \multicolumn{6}{|c|}{ T2 (\% within T2) } \\
\hline PC & $128(82.6 \%)$ & $13(8.4 \%)$ & $4(2.6 \%)$ & $5(3.2 \%)$ & $5(3.2 \%)$ \\
\hline C & $20(18.3 \%)$ & $63(57.8 \%)$ & $13(11.9 \%)$ & $10(9.2 \%)$ & $3(2.8 \%$ \\
\hline
\end{tabular}

Note, $\mathrm{PC}=$ precontemplation, $\mathrm{C}=$ contemplation, $\mathrm{PR}=$ preparation, $\mathrm{A}=$ action, $\mathrm{M}=$ maintenance. ${ }^{b} \mathrm{~T} 2$ is 53 days after $\mathrm{T} 1 ; \mathrm{T} 3$ is 106 days after T1.

"Figures represent number of pretest participants, with the percentage of pretest participants in parentheses.

Forward stage transitions out of precontemplation and contemplation are printed in italic.

\section{Cross-sectional analyses}

Table 2.2 shows the differences between stages of change in terms of pros, cons, self-efficacy, and fruit intake at T1, T2, and T3. At all three points in time, the pros of eating two servings of fruit each day were significantly lower in precontemplation than in all other stages. At $\mathrm{Tl}$, the pros were also lower in contemplation than in preparation.

At all three measurements, the cons of eating two servings of fruit every day were significantly higher in precontemplation and contemplation than in maintenance. At $\mathrm{T} 1$ and $\mathrm{T} 2$, the cons were also higher in preparation than in maintenance, and at $\mathrm{T} 1$ cons were higher in action than in maintenance. At all 
points in time, self-efficacy scores were significantly higher in maintenance than in all other stages, and higher in action than in precontemplation and contemplation. At $\mathrm{T} 1$ and $\mathrm{T} 2$, but not at $\mathrm{T} 3$, respondents in action also had higher self-efficacy scores than respondents in preparation.

Also at all three measurements, fruit intake was higher in maintenance than in precontemplation, contemplation, and preparation, and higher in action than in precontemplation and contemplation. At T1, fruit intake was higher in preparation than in precontemplation, and at $\mathrm{T} 2$ fruit intake was higher in action than in preparation.

Table 2.2 Mean scores on possible predictors of stage transition for respondents in different stages of change and the results of analyses of variance with Scheffé post-hoc test testing for differences between the stages

\begin{tabular}{|c|c|c|c|c|c|c|c|}
\hline $\begin{array}{l}\text { Variable } \\
(-2 \text { to } 2)\end{array}$ & PC & $c$ & PR & $\mathbf{A}$ & $M$ & F value & $\begin{array}{l}\text { Scheffé multilple- } \\
\text { comparison test }\end{array}$ \\
\hline $\mathrm{T1}(\mathrm{N}=648)$ & $n=202$ & $n=137$ & $\mathrm{n}=84$ & $n=56$ & $n=169$ & & \\
\hline Pros & 0.15 & 0.45 & 0.70 & 0.56 & 0.55 & $20.23^{* * * *}$ & $\begin{array}{l}\mathrm{PC}<\mathrm{C}, \mathbb{P R}, \mathrm{A}, \mathrm{M} ; \\
\mathrm{C}<\mathrm{PR}\end{array}$ \\
\hline Cons & -0.10 & -0.06 & -0.07 & -0.18 & -0.48 & $1.2 .80^{* * * *}$ & $\mathrm{M}<\mathrm{PC}, \mathrm{C}, \mathrm{PR}_{r} \mathrm{~A}$ \\
\hline Self Efficacy & -0.26 & -0.25 & -0.11 & 0.40 & 1.12 & $100.85^{* * *}$ & $\mathrm{PC}, \mathrm{C}, \mathrm{PR}<\mathrm{A}<\mathrm{M}$ \\
\hline Fruilt intake & 172 & 203 & 261 & 323 & 371 & $52.39 * * * *$ & $\begin{array}{l}\mathrm{PC}, \mathrm{C}<\mathrm{A}, \mathrm{M} ; \\
\mathrm{PC}<\mathrm{PR} ; \mathrm{PR}<\mathrm{M}\end{array}$ \\
\hline $\mathrm{T} 2(\mathrm{~N}=592)$ & $n=179$ & $n=126$ & $n=49$ & $n=79$ & $n=159$ & & \\
\hline Pros & -0.03 & 0.32 & 0.53 & 0.59 & 0.53 & $24.68^{* * * *}$ & $P C<C, P R A, M$ \\
\hline Cons & -0.04 & -0.13 & -0.09 & -0.24 & -0.44 & $8.70^{2+4 * *}$ & $\mathrm{M}<\mathrm{PC}, \mathrm{C}, \mathrm{PR}$ \\
\hline Self Efficacy & -0.33 & -0.28 & -0.15 & 0.35 & 1.11 & $99.35^{* * * * 4}$ & $P C, C, P R<A<M$ \\
\hline Fruit intake & 156 & 184 & 190 & 325 & 366 & $65.42^{* * 4}$ & $\mathrm{PC}, \mathrm{C}, \mathrm{PR}<\mathrm{A}, \mathrm{M}$ \\
\hline $\mathrm{T} 3(\mathrm{~N}=570)$ & $n=180$ & $n=98$ & $n=65$ & $n=77$ & $n=150$ & & \\
\hline Pros & -0.02 & 0.41 & 0.59 & 0.54 & 0.63 & $32.10^{* * * *}$ & $\mathrm{PC}<\mathrm{C}, \mathrm{PR}_{\mu}, \mathrm{A}, \mathrm{M}$ \\
\hline Cons & -0.12 & -0.08 & -0.18 & -0.19 & -0.42 & $5.36 * * *$ & $\mathrm{M}<\mathrm{PC}, \mathrm{C}$ \\
\hline Self Etficacy & -0.31 & -0.37 & 0.11 & 0.30 & 1.01 & $70.00 * *$ & $\mathbb{P C}_{x} \mathbb{C}<\mathrm{PR}, \mathrm{A}<\mathrm{M}$ \\
\hline Fruit inlake & 153 & 169 & 244 & 306 & 360 & $57.65^{* * *}$ & $\begin{array}{l}\mathrm{PC}, \mathrm{C}<\mathrm{PR}, \mathrm{A}, \mathrm{M} ; \\
\mathrm{PR}<\mathrm{M}\end{array}$ \\
\hline
\end{tabular}

Note. a Fruit intake in grams a day; Significant at ${ }^{*} p<05,{ }^{* *} p<.01$, **** $p<.001$

$\mathrm{PC}=$ precontemplation, $\mathrm{C}=$ contemplation, $\mathrm{PR}=$ preparation, $\mathrm{A}=$ action, $\mathrm{M}=$ maintenance. 


\section{Longitudinal analyses}

\section{Forward stage transition out of precontemplation}

Forward stage transition out of precontemplation was predicted by higher pros in both intervals and by higher self-efficacy and higher fruit intake between T2 and T3 (Table 2.3). Multivariate logistic regression showed that between T2 and $\mathrm{T} 3$, only pros was a significant predictor of forward stage transition out of precontemplation.

Table 2.3 Odds ratio (OR) and $95 \%$ confidence intervals (CI) derived from simple and multiple binary logistic regression analyses to identify predictors of forward stage transition out of the precontemplation stage for two time intervals

\begin{tabular}{|c|c|c|c|c|}
\hline \multirow[b]{2}{*}{ Simple } & \multicolumn{2}{|c|}{ T1-T2 $(N=169)^{a}$} & \multicolumn{2}{|c|}{$T_{2}-T_{3}(N=153)$} \\
\hline & OR & $95 \% \mathrm{Cl}$ & OR & $95 \% \mathrm{Cl}$ \\
\hline Pros & $2.00^{*}$ & $1.05-3.81$ & $5.48^{* * *}$ & $2.09-14.32$ \\
\hline Cons & 0.88 & $0.51-1.53$ & 1.05 & $0.56-1.96$ \\
\hline Self-efficacy & 1.41 & $0.96-2.09$ & $2.12^{* * *}$ & $1.26-3.56$ \\
\hline Fruit intake & 1.002 & $1.000-1.005$ & $1.004^{* *}$ & $1.001-1.006$ \\
\hline \multicolumn{5}{|l|}{ Multiple } \\
\hline Pros & 1.95 & $0.96-3.95$ & $4.21^{* * *}$ & $1.51-11.80$ \\
\hline Cons & 0.74 & $0.39-1.38$ & 1.05 & $0.47-2.34$ \\
\hline Self-efficacy & 1.22 & $0.80-1.86$ & 1.69 & $0.94-3.04$ \\
\hline
\end{tabular}

Note. Significant at $* p<.05_{*}^{* *} p<.01_{*}^{* * *} p<.001$

a T1-T2 $=$ Precontemplators at T1 who progressed out of or remained stable in precontemplation 53 days later at $\mathrm{T} 2 ; \mathrm{T} 2-\mathrm{T} 3=$ Precontemplators at $\mathrm{T} 2$ who progressed out of remained stable in precontemplation 53 days later at $\mathrm{T} 3$.

Forward stage transition out of contemplation

Between T2 and T3, forward stage transition out of contemplation was predicted. only by higher self-efficacy scores in simple as well as multiple logistic regression analyses (Table 2.4 ). 
Table 2.4 Odds ratio (OR) and $95 \%$ confidence intervals (CI) derived from simple and multiple binary logistic regression anallyses to identify predictors of forward stage transition out of the contemplation stage for two time intervals

\begin{tabular}{|c|c|c|c|c|}
\hline \multirow[b]{2}{*}{ Simple } & \multicolumn{2}{|c|}{$T 1-T 2(N=169)^{a}$} & \multicolumn{2}{|c|}{$\mathrm{T} 2-\mathrm{T} 3(\mathrm{~N}=153)$} \\
\hline & OR & $95 \% \mathrm{C}$ & OR & $95 \% \mathrm{Cl}$ \\
\hline Pros & 188 & $0.95-3.73$ & 1.29 & $0.62-2.70$ \\
\hline Cons & 0.84 & $0.48-1.47$ & 144 & $0.70-2.97$ \\
\hline Sex-efficacy & 1.40 & $0.76-2.57$ & $2.13^{*}$ & $1.06-4.30$ \\
\hline Fruit intake & 0.999 & $0.995-1.002$ & 1.002 & $0.999-1.006$ \\
\hline \multicolumn{5}{|l|}{ Multiple } \\
\hline Pros & 1.99 & $0.98-4.04$ & 1.01 & $0.45-2.28$ \\
\hline Cons & 0.72 & $0.39-1.36$ & 1.68 & $0.72-3.90$ \\
\hline Self-efficacy & 125 & $0.66-2.39$ & $2.31^{*}$ & $1.10-4.84$ \\
\hline
\end{tabular}

Note. Significant at * p<.05

${ }^{\mathrm{T}} \mathrm{T} 1-\mathrm{T} 2=$ Contemplators at $\mathrm{T} 1$ who who progressed out of or remained stable in contemplation 53 days later at $\mathrm{T} 2 ; \mathrm{T} 2-\mathrm{T} 3=$ Contemplators at $\mathrm{T} 2$ who progressed out of or remained stable in contemplation 53 days later at T3.

\section{Discussion}

The aim of the present study was to examine associations between pros, cons, self-efficacy, stages of change, and forward stage transitions related to fruit intake using cross-sectional and longitudinal methods. Pros, fruit intake, and self-efficacy predicted forward stage transition out of precontemplation. Selfefficacy predicted forward stage transition out of contemplation. No support was found for cons as a stage transition determinant. To a large extent, results from longitudinal analyses were similar to cross-sectional results, except for selfefficacy: no differences between early stages in self-efficacy scores were found, while self-efficacy scores predicted these early stage transitions.

Our cross-sectional findings are comparable to results from previous studies, and in line with predictions from the TTM. Pros were higher in contemplation than in precontemplation, which is consistent with results of $\mathrm{Ma}$ et al. (2002) and Horacek et al. (2002) and these results are all in line with predictions from the TTM (Prochaska, 1994). Longitudinally, our results also provided some evidence that the perceived pros of fruit intake may predict progress out of precontemplation. Plotnikoff et al. (2001) reported similar results for exercise stage transitions out of both precontemplation and contemplation. Also, in smoking cessation, some evidence has been reported in support of the role of pros of quitting in forward stage transition out of precontemplation (De 
Vries \& Mudde, 1998; Dijkstra et al., 2003; Velicer et al., 1999) as well as out of contemplation (De Vries \& Mudde, 1998; Velicer et al., 1999).

Remarkably, our results indicated that cons were not relevant in stage transitions, either in the cross-sectional or the longitudinal analyses. Whereas the TTM predicts a decrease in cons between the contemplation and action stages, this was not confirmed in the present study or by earlier studies on the same topic (Horacek et al., 2002; Ma et al., 2002). All of these studies, however, including the present research, consistently found the lowest cons in maintenance. Earlier longitudinal studies have further shown similar results concerning the role of cons in stage transitions: in the exercise domain, no support was found for cons as a relevant predictor of stage transition out of precontemplation or contemplation (Plotnikoff et al., 2001). The notion that cons are relevant for early stage transitions is largely based on research on smoking cessation stages of change. For example, De Vries and Mudde (1998) found a relationship between cons and precontemplation forward stage transition, and Velicer et al. (1999) found cons to be related to forward stage transitions from precontemplation and contemplation, although the effect sizes were small. An explanation for the difference in results for fruit intake might be that people do not perceive the cons of eating fruit as very important. Even in precontemplation, respondents rated the cons as less important than the pros in their decision to eat two servings of fruit each day. In addition, although our con-scalle was based on earlier studies and pretested in the Dutch situation, the potential cons listed in the questionnaire may not have been fully representative of the cons perceived by the participants.

The higher self-efficacy scores in maintenance than in action, and higher self-efficacy scores in action than in preparation is similar to earlier research on fruits and vegetables and predictions from the TTM (Horacek et al., 2002; Plummer et al., 2001). People in the action and maintenance stages have changed their behavior, and in these stages self-efficacy may be based on experience rather than on the perception of a behavior. Few differences in self-efficacy were found between pre-action stages, and self-efficacy was low in these stages. Yet, our longitudinal analyses provided some indications that self-efficacy may be relevant to progress through these pre-action stages, since self-efficacy predicted forward stage transition out of precontemplation and contemplation in one of the two time intervals that was studied. The possible role of self-efficacy in changes in early motivational stages is consistent with self-efficacy theory (Bandura, 1986; Schwarzer, 1992a). Previous studies have shown similar results for self-efficacy in precontemplation and contemplation forward stage transition in exercise (Courneya et al., 2001; Plotnikoff et al., 2001). In smoking cessation, however, no support was found for self-efficacy as a relevant factor in stage 
transition out of precontemplation and contemplation (De Vries \& Mudde, 1998; Dijkstra et al., 2003).

Fruit intake was higher in action/maintenance than in the pre-action stages. This confirms the results of past research and predictions from the TTM (Brug et al „1 1997; Campbell et al., 1998; Van Duyn et al., 2001). Fruit intake was found significantly to predict stage transition out of precontemplation in both time intervals, although the odds ratios were small. This might indicate that people, who progress through the early stages of change, may have made small initial behavior changes but do not yet meet the recommendations (Prochaska \& Velicer, $1997 \mathrm{a})$. It might also indicate that precontemplators whose current diet is rather close to the recommendations are somewhat more ready for change than other precontemplators. For example, precontemplators eating one serving of fruit each day may progress more easily than precontemplators whose current diet does not contain any servings of fruit.

There are limitations to the data reported here. The existing Internet panel sampling frame may not have been representative of the Dutch population and, thus, may have reduced external validity. Compared to the Dutch population at large, higher educated people (36\% vs. 29\%) and respondents younger than 30 (36\% vs. $21 \%$ ) were overrepresented; lower educated people $(16 \%$ vs. $27 \%)$ and respondents over 55 years old $(15 \%$ vs. $70 \%)$ were underrepresented; and the prevalence of eating less than two servings of fruit a day (57\% vs. 70\%) was lower (Dutch Nutrition Centre, 1998; Statistics Netherlands, 2003). Using an Internet panel, however, is a convenient and attractive method to have repeated measurements within short time intervals. Research on web-based surveys has shown that rather high response rates can be established, with the majoxity of responses returned in a 24 - to 48 -hour time period following the questionnaire completion request (Couper, 2000).

To conclude, within the constraints posed by the sampling frame, the present study provides evidence that pros and fruit intake are relevant in forward stage transition out of precontemplation. Inconsistent with predictions from the TTM, we did not find support for cons as a relevant variable in forward stage transition. Support was found for self-efficacy as a rellevant variable in forward stage transition from precontemplation and contemplation. In order to be a valid stage model, stages of change should be qualitatively different. This means that determinants should be relevant at different stages, i.e, determinants should be stage-specific. If this assumption cannot be confirmed, health behavior change might follow a continuous process rather than a staging process (Weinstein et al., 1998). It should be noted that self-efficacy might not be a stagespecific transition determinant, and more research is needed into the qualitative difference of stages of change. 


\section{Implications for health promotion practice or research}

Within the limitations posed by the sampling frame, this study seems to indicate that pros, self-efficacy and fruit intake might determine forward stage transition out of precontemplation, and that forward transition out of contemplation might be determined by self-efficacy. Combined with the results of previous studies, there seems to be partial support for the TTM, although the evidence suggests that determinants might not always be stage-specific. If this assertion holds true, results can be used to develop and evaluate interventions tailored to the fruitrelated precontemplation and contemplation stages. However, the study needs to be replicated in a more representative sample and more research is needed into the qualitative difference of stages of change. 


\title{
Chapter 3 \\ Do the Transtheoretical processes of change predict transitions in stages of change for fruit intake?
}

\begin{abstract}
According to the Transtheoretical Model (TTM), processes of change are hypothesized to predict transitions in stages, though the processes of change are the TTM's least studied component. In a longitudinal study, it is explored whether the processes of change do predict stage transitions in fruit intake. A random sample of an existing Internet research panel, resulted in a cohort of 735 adults (response rate 62\%), who were examined three times with electronic questionnaires assessing stages of change, processes of change and fruit intake at $\mathrm{T} 1(\mathrm{~N}=648$; response rate $88 \%), 53$ days later at $\mathrm{T} 2(\mathrm{~N}=592$; response rate $81 \%)$, and another 53 days later at $\mathrm{T} 3(\mathrm{~N}=570$; response rate $78 \%)$. Cross-sectional differences were found for the processes of change between precontemplation and all further stages. Experiential as well as behavioral processes increased from precontemplation to action with similar patterns. Both experiential and behavioral processes predicted forward transition out of precontemplation and forward transition into action, while only behavioral processes predicted forward transition out of contemplation. In conclusion, more frequent application of processes of change seems associated with more advanced stages of change, although the two-factor structure of experiential and behavioral processes may not be distinguishable for fruit intake.
\end{abstract}

A revised version of this chapter is resubmitted for publication as: De Vet, E., De Nooijer, J., De Vries, N. K., Brug, J. Do the Transtheoretical processes of change predict transitions in stage of change for fruit intake? 


\section{Introduction}

Ample fruit intake is considered to be an important everyday health behavior that may contribute to the prevention of obesity (Rolls et al, 2004), cardiovascular diseases (Srinath Reddy \& Katan, 2004), and certain cancers (Key et al., 2004), whereas in most countries, including the US and most countries in Europe, a majority of people do not meet the guidelines for sufficient fruit intake (Serdula et al., 2004, Van der Wilk \& Jansen, 2005). In the Netherlands, approximately $70 \%$ of the population eat less than the recommended minimum amount of two servings (250 grams) of fruit per day (Van Oers, 2002).

It is important to understand why people do not consume adequate amounts of fruit and how fruit intake can be increased. Health behavior theories can be helpful in increasing this knowledge. A widely used theory is the Transtheoretical Model of Behavior change (TTM). The TTM conceptualizes when and how behavior change occurs, and postulates that behavior change is a dynamic process, which involves movement through a sequence of discrete, qualitatively distinct stages (Prochaska \& DiClemente, 1983). The core organizing construct of the TTM is the Stages of Change construct, which represents a temporal dimension and distinguishes five stages of change, thus indicating when individuals change: precontemplation (no intention to change behavior within the next six months), contemplation (intention to change behavior within the next six months), preparation (intention to change behavior within 30 days), action (people changed from unhealthy to healthy behavior within the past six months), and maintenance (maintenance of the behavior change for more than six months; Prochaska \& Velicer, 1997a). People may move forward and backward through the stages of change. Behavior change is usually not a linear process but rather cyclical in nature, though in progression towards maintenance most people will follow the sequence as outlined by the TTM.

The TTM postulates that movement through the stages of change is associated with the engagement in processes of change. The processes of change are the independent variables that render how people proceed to change their behaviors and are therefore assumed to provide good opportunities for intervention programs (Prochaska \& Velicer, 1997a; Prochaska, Velicer, DiClemente, \& Fava, 1988). The processes of change reflect covert and overt activities that individuals use to move from stage to stage and finally to modify their problem behaviors (Prochaska et al, 1988). At each of the stages individuals use different processes to enable movement to a next stage (see Table 3.1). The TTM distinguishes ten separate processes of change, which are grouped in a higher order structure with two factors (Table 3.1, Prochaska \& Velicer, 1997a). 
Table 3.1 Processes of change ( $(\mathrm{f} .$, Prochaska \& Velicer, 1997a) and questionnaire items

\begin{tabular}{|c|c|c|c|c|}
\hline \multicolumn{2}{|l|}{. } & Process & \multirow{2}{*}{$\begin{array}{l}\text { Description } \\
\text { Increase awareness about the } \\
\text { causes, consequences, and } \\
\text { cures for a particular } \\
\text { problem behavior. }\end{array}$} & \multirow{2}{*}{$\begin{array}{l}\text { ltem } \\
\text { I'm interested in } \\
\text { information about } \\
\text { eating fruits. }\end{array}$} \\
\hline$\frac{5}{8}$ & \multirow{5}{*}{ 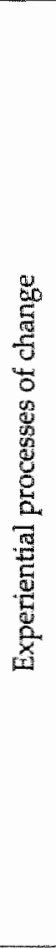 } & Consciousness raising & & \\
\hline $\begin{array}{l}\frac{5}{8} \\
\frac{8}{5} \\
\frac{1}{5} \\
\frac{8}{2} \\
\frac{1}{2}\end{array}$ & & Dramatic relief & $\begin{array}{l}\text { Produce increased emotional } \\
\text { experiences followed by } \\
\text { reduced affect if appropriate } \\
\text { action can be taken. }\end{array}$ & $\begin{array}{l}\text { I an afraid of the } \\
\text { consequences to my } \\
\text { health if I do not eat at } \\
\text { least wo servings of } \\
\text { fruit each day. }\end{array}$ \\
\hline 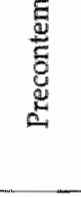 & & $\begin{array}{l}\text { Environmental } \\
\text { reevaluation }\end{array}$ & $\begin{array}{l}\text { Combine both affective and } \\
\text { cognitive assessments of } \\
\text { how presence or absence of a } \\
\text { personal habit affects one's } \\
\text { social environment. }\end{array}$ & $\begin{array}{l}\text { I realize that when I } \\
\text { eat less than two } \\
\text { servings a day, I may } \\
\text { get ill and be a burden } \\
\text { to others. }\end{array}$ \\
\hline 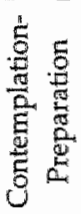 & & Self- reevaluation & $\begin{array}{l}\text { Both cognitive and affective } \\
\text { assessments of one's self- } \\
\text { image with and without a } \\
\text { particular unhealthy habit. }\end{array}$ & $\begin{array}{l}\text { I belief that eating two } \\
\text { servings of fruit each } \\
\text { day will make me a } \\
\text { happier and healthier } \\
\text { person. }\end{array}$ \\
\hline \multirow{2}{*}{ 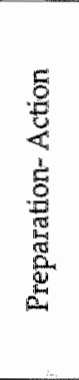 } & & Social liberation & $\begin{array}{l}\text { Realizing that sociall morms } \\
\text { are changing in the direction } \\
\text { of supporting the healthy } \\
\text { behavior change. }\end{array}$ & $\begin{array}{l}\text { I am aware that more } \\
\text { and more people } \\
\text { know that eating two } \\
\text { servings of fruit each } \\
\text { day is good for them. }\end{array}$ \\
\hline & \multirow{5}{*}{ 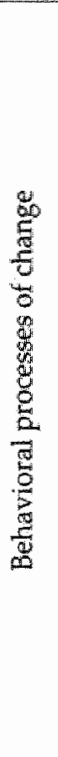 } & Self-liberation & $\begin{array}{l}\text { Believing that one can } \\
\text { change and the commitment } \\
\text { to change. }\end{array}$ & $\begin{array}{l}\text { tell inyself that I can } \\
\text { eat at least two } \\
\text { servings of fruit each } \\
\text { day, if I try hard } \\
\text { enough. }\end{array}$ \\
\hline \multirow{4}{*}{ 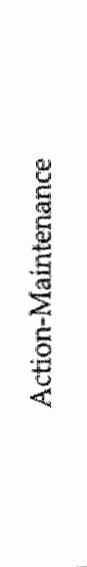 } & & Counterconditioning & $\begin{array}{l}\text { Learning of healthier } \\
\text { behavior that can replace } \\
\text { problem behavior. }\end{array}$ & $\begin{array}{l}\text { Instead of a sinack I } \\
\text { takte a serving of frutut }\end{array}$ \\
\hline & & Stimulus control & $\begin{array}{l}\text { Removing cues for } \\
\text { unhealthy habits and add } \\
\text { prompts for healthier } \\
\text { alternatives. }\end{array}$ & $\begin{array}{l}\text { do things that remind } \\
\text { me of eating at least } \\
\text { two servings of fruit } \\
\text { each day. }\end{array}$ \\
\hline & & $\begin{array}{l}\text { Reinforcement } \\
\text { management }\end{array}$ & $\begin{array}{l}\text { Reinforcements for taking } \\
\text { steps in al particular } \\
\text { direction. }\end{array}$ & $\begin{array}{l}\text { Other people reward } \\
\text { me when I eat at least } \\
\text { two serwings of fruit } \\
\text { eadh day. }\end{array}$ \\
\hline & & Helping relationships & $\begin{array}{l}\text { Positive, supportive } \\
\text { relationships that facilitate } \\
\text { change. }\end{array}$ & $\begin{array}{l}\text { It have someone who } \\
\text { encowrages me to eat } \\
\text { two servings of fruit } \\
\text { each day. }\end{array}$ \\
\hline
\end{tabular}


The ten processes of change are grouped into the two factors, i.e.s. experiential and behavioral processes, since individuals are considered to use multiple processes at the same time (Prochaska et al., 1988). The first factor reflects the experiential processes, which are cognitive, affective or evaluative in nature, such as feelings, thoughts and experiences. Examples of the experiential processes are consciousness-raising, dramatic relief, self-reevaluation, environmental re-evaluation and social liberation (see Table 3.1 for a description of the separate processes; Prochaska \& Velicer, 1997a). The second factor incorporates the behavioral processes of change, which reflect behavioral or active strategies that are used to change behavior. Examples of these behavioral processes are self-liberation, counterconditioning, stimulus control, reinforcement management and helping relationships (Prochaska \& Velicer, 1997a). Selfliberation (beljeving in one's ability to change and the commitment to change) might be regarded as an experiential process, since strictly speaking selfliberation does not involve a behavioral action. However, this process tends to cooccur with the behavioral processes and is therefore grouped together with the behavioral processes (Prochaska, DiClemente, \& Norcross, 1992).

These sets of processes are used differentially across stages of change. The TTM hypothesizes that experiential processes are most relevant for individuals in the contemplation and preparation stages since in those stages cognitive change is required, whereas behavioral processes of change are expected to peak in the action stage as actual behavior change occurs (Perz, DiClemente, \& Carbonari, 1996; Prochaska et al., 1988; Prochaska, Velicer, Guadagnoli, Rossi, \& DiClemente, 1991).

Unlike the stages of change concept which has been applied to a broad range of behaviors, the processes of change have been largelly neglected in interventions and research based on the TTM for many health behaviors, including nutrition (Horacek et al, 2002; Kloek, Van Lenthe, Van Nierop, \& Mackenbach, 2004; Ling \& Horwath, 2001; Ma, Betts, Horacek, Georgiou, \& White, 2003; Van Duyn et al, 2001). This is remarkable since the TTM explicitly proposes that the processes of change guide transitions in stages. In a study that did look at processes of change related to fruit intake, Greene and colleagues (2004) found in a sample of older adults (above 60 years) that all processes of change were used more often in contemplation/preparation than in precontemplation. In action/maintenance, two experiential processes (selfreevaluation and social liberation) and two behavioral processes (counterconditioning and stimulus control) were more frequently used as compared to the pre-action stages. In a cross-sectional survey on fruit and vegetable intake among male college students, Fontes de Oliveira and colleagues (2005) found that processes were used more often in preparation and action/maintenance stages than in precontemplation/contemplation combined, with the exception of stimulus control. Stimulus control did not differ between 
pre-action stages. Further, no differences in process use between preparation and action/maintenance stages were found (Fontes de Oliveira et al., 2005). Similar increases in process use were found through the stages of dietary fat reduction, with lowest process use in precontemplation and some processes peaking in action (Bowen et al., 1994; Greene et al., 1999).

From these studies it seems likely that increased process use is indeed associated with more advanced stages of change, finally resulting in healthy dietary behavior. However, to date no longitudinal studies have been conducted that actually test whether these processes of change predict shifts in stages of change for fruit intake as proposed by the TTM. The objective of the present study is to explore 1) whether results from cross-sectional studies could be replicated in a Dutch adult sample, 2) whether the processes of change predict stage transitions in fruit intake, longitudinally, and 3) whether the relevance of these processes confirms TTM hypotheses, with more cognitive strategies of importance from precontemplation to preparation and the behavioral strategies of importance from preparation to action.

\section{Method}

\section{Design, participants and procedures}

The study presents results from secondary data analyses of a larger longitudinal study on stages of change and fruit intake. The study design is described in chapter 2 (De Vet, De Nooijer et al., 2005a). In brief, a cohort of 735 adults randomly selected from a Dutch Internet research panel were asked three times to complete an electronic questionnaire, i.e., at the start of the present study (T1), 53 days later (T2) and 106 days after T1 (T3). A baseline (T0) questionnaire was used to invite potential respondents, to describe the cohort on demographic characteristics and fruit intake, and to classify respondents into stages of change. For $\mathrm{T} 1, \mathrm{~T} 2$ and $\mathrm{T} 3$ the electronic questionnaires were identical and included items measuring stage of change, processes of change, and a food frequency questionnaire to assess fruit intake. A time interval of two weeks was used to assess test-retest reliability for these variables.

The data were analyzed cross-sectionally for three measurements separately $(\mathrm{T} 1, \mathrm{~N}=648$, response rate $88 \%, \mathrm{~T} 2, \mathrm{~N}=592$, response rate $81 \%$; $\mathrm{T} 3, \mathrm{~N}=$ 570 , response rate $78 \%$ ), and longitudinally for the two equal time intervals of 53 days between these three measurements, $\mathrm{T} 1-\mathrm{T} 2(\mathrm{~N}=552$, response rate $75 \%)$ and $\mathrm{T} 2-\mathrm{T} 3(\mathrm{~N}=518$, response rate $70 \%)$, to compare differences between stages of change and predictors of stage transitions. Three measurements and, thus, two 
time intervals were analyzed in order to try to replicate the results within the study.

In total, $488(66 \%)$ respondents completed the three questionnaires. Respondents who did not complete all three questionnaires were significantly younger $(\mathrm{F}(1,735)=25.90, p<.001)$ and are more often female $\left(\chi^{2}(1, N=735)=\right.$ $5.00 ; p<.05)$ than those who completed all three questionnaires. No differences between completers and non-completers were found with respect to level of education, ethnicity, stage of change or fruit intake.

\section{Measures}

Fruit intake was measured using a 14-item food frequency questionnaire (FFQ) assessing the frequency of consumption during the previous month ("never/less than once a month" to "7 days a week") and assessing the amount of fruit consumed (pieces or small bowls [for small fruit such as berries] per day) with separate questions for the most common fruits in the Netheriands, i.e., citrus fruit, apples and pears, bananas, freshly squeezed or unsweetened fruit juice, tangerines, and for other fruit including preserved fruit. The FFQ has been validated as compared with a 7 -day dietary record $(r=.51 ; p<.001$; Van Assema et al., 2002) and biomarkers for fruit, i.e., blood levels of total carotenoids, vitamin $\mathrm{C}$, and beta-cryptoxanthin $(r=.34, r=.27$, and $r=.42$, respectivelly; all $p<.001$; Bogers et al., 2004). Test-retest reliability with a two-week interval showed a Pearson correlation of $r=.68, p<.001$.

Stage of change was operationalized by a single-item algorithm consistent with Armitage and Arden (2002) and Laforge et al. (1999) and adapted to fruit intake by asking respondents to select from five statements, each representing a stage of change, the most applicable to their personal situation: "I do not eat at least two servings of fruit every day and I do not intend to change this within the next six months" [precontemplation], "I do not eat at least two servings of fruit every day, but I intend to change this within the next six months" [contemplation], "I do not eat at least two servings of fruit every day, but I intend to change this within the next month" [preparation] "I eat at least two servings of fruit every day, and I have started doing this in the last six months" [action] "I eat at least two servings of fruit every day, and $I$ have done so for more than six months" [maintenance]. Test-retest reliability for stages of change with a twoweek interval showed a Kappa of $.57(p<.001)$.

Processes of change were assessed by asking respondents to indicate how often they had experienced the strategy described in ten statements, each statement representing a process of change, using a 5-point Likert scale, ranging from 1 (never) to 5 (repeatedly) consistent with instructions from the TTM (Fava, Velicer, \& Prochaska, 1995). No processes of change questionnaire for fruit intake 
was available at the time of study, so processes of change questionnaires for other behaviors provided input for the present questionnaire and items were adapted to fruit intake whenever appropriate (McKenna \& Francis, 2003; Prochaska et al., 1988; Rossi et al., 1994; see Table 3.1 for the wording of the items). The questionnaire was pretested among Dutch adults. Based on the TIM, aggregate variables for the experiential (consciousness-raising, dramatic relief; selfreevaluation, environmental re-evaluation and social liberation) and behavioral (self-liberation, counterconditioning, stimulus control, reinforcement management and helping relationships) processes were computed (Prochaska \& Velicer, 1997a). Cronbach's $\alpha$ for the experiential and behavioral processes of change were .78 and .62 respectively. Test-retest reliability with a two-week interval showed a Pearson correlation of $r=.71, p<.01$ for the experiential processes and $r=.65_{r} p<$ .01 for the behavioral processes.

Demographic variables included age, sex, level of education, and ethnicity and were only assessed at TO.

\section{Analyses}

Descriptive statistics were used to describe the study population at baseline and stage transitions out of precontemplation and contemplation for both time intervals.

To examine cross-sectional differences in the experiential and behavioral processes of change between stages of change at T1, T2, and T3, ANOVA with Scheffé multiple-comparison tests were used. At $\mathrm{T} 1$ the same analyses were conducted for all ten processes of change separately.

Since the sample size was not sufficient to examine all possible patterns of stage change, for both time intervals stage transitions were transferred into a dichotomous variable for precontemplation $(1=$ precontemplators who progressed at least one stage; $0=$ precontemplators who remained stable), contemplation $(1=$ contemplators who progressed at least one stage; $0=$ contemplators who remained stable or regressed), action $(1=$ respondents in one of the pre-action stages who progressed into the action stage; $0=$ respondents who remained in one of the pre-action stages). The present sample comprised few respondents who progress from preparation to action (between $T 1$ and $T 2, n=25$ and between $\mathrm{T} 2$ and $\mathrm{T} 3, \mathrm{n}=10$ ). In order to gain insight into the preparationaction transition, all respondents in the pre-action stages at $\mathrm{T} 1$ who were in action at T2 were grouped together. The TTM assumes that respondents pass through all distinct stages. So the respondents who reach the action stage from precontemplation and contemplation are assumed to have passed through the preparation stage as well. Research on fruit intake confirmed this stage model assumption (De Vet, De Nooijer, De Vries, \& Brug, 2005b). 
In line with recommendations and earlier prospective analyses on processes of change (Herzog et al., 1999; Segan, Borland, \& Greenwood, 2004; Sutton, 2000a), logistic regression analyses were conducted with stage transition between $\mathrm{T} 1$ and $\mathrm{T} 2$ as the dependent variable and the processes of change at $\mathrm{T} 1$ as independent variables to explore and test predictors of forward stage transition from precontemplation and contemplation, and from pre-action stages into action. First, analyses were conducted for the aggregates of experiential and behavioral processes, separately. When the aggregate of experiential or behavioral processes significantly contributed to the prediction of a stage transition, the effect of the ten separate processes was examined. The exact same analyses were conducted for $\mathrm{T} 2-\mathrm{T} 33$ to replicate the results found for the T1-T2 interval.

Finally, univariate linear regression analyses were conducted with fruit intake at $T_{2}$ as the dependent variable and the stage transitions from precontemplation, contemplation, and to action between $\mathrm{T} 1$ and $\mathrm{T} 2$ as the separate independent variables. The analyses were repeated with fruit intake at T3 as the dependent variable, and the three stage transitions between T2 and T3 as the independent variables.

For all statistical analyses the alpha level was set at .05 .

\section{Results}

\section{Participants}

The mean age of participants was $37.5(\mathrm{SD}=14.1)$ years, and women and men were equally represented ( $51 \%$ vs. $49 \%$ ). Of the respondents, $17 \%, 48 \%$, and $36 \%$ reported a low, medium and high level of education, respectively. Most respondents were of Dutch origin $(90 \%)$. Mean fruit intake was $260(\mathrm{SD}=163$ ) grams per day, and $57 \%$ of the respondents ate less fruit than the recommended minimum intake level of 250 grams a day. At baseline (TO), 242 (33\%) respondents were in precontemplation, $157(21 \%)$ in contemplation, $104(14 \%)$ in preparation, $49(7 \%)$ in action, and $183(25 \%)$ in maintenance. 


\section{Cross-sectional analyses}

\section{Experiential processes of change}

At all three time points, respondents in precontemplation applied the experiential processes less often than respondents in all other stages (see Table 3.2). At T1, respondents in contemplation also applied the experiential processes less often than respondents in preparation. At both $\mathrm{T} 1$ and $\mathrm{T} 2$ experiential processes were applied most often in preparation, while at T3, experiential process use peaked in maintenance. For the separate processes of change, similar consistent patterns were found with consciousness raising, dramatic relief, environmental reevaluation, self-reevaluation and social liberation being applied least often in precontemplation. Consciousness-raising was further applied less often in contemplation than in preparation.

\section{Behavioral processes of change}

At all three time points, respondents in precontemplation applied the behavioral processes less often than respondents in all other stages. Further, at all three time points, behavioral processes were applied more often in preparation and action than in maintenance. Respondents in contemplation applied behavioral processes less often than respondents in preparation at $\mathrm{T} 1$ and less often than respondents in action at T2 and T3. At T1, behavioral processes peaked in preparation, but at $\mathrm{T} 2$ and $\mathrm{T} 3$ these processes peaked in the action stage. Cross-sectional analyses of the separate processes at $\mathrm{T} 1$ showed that counterconditioning was applied less often in precontemplation and contemplation than in action and maintenance, and also less often in precontemplation than in preparation. Helping relationships, reinforcement management, self-liberation and stimulus control were applied less in precontemplation than in contemplation and preparation. Precontemplators also applied helping relationships less often than maintainers, while for reinforcement management, self-liberation and stimulus control, no differences were found between precontemplation and maintenance. 


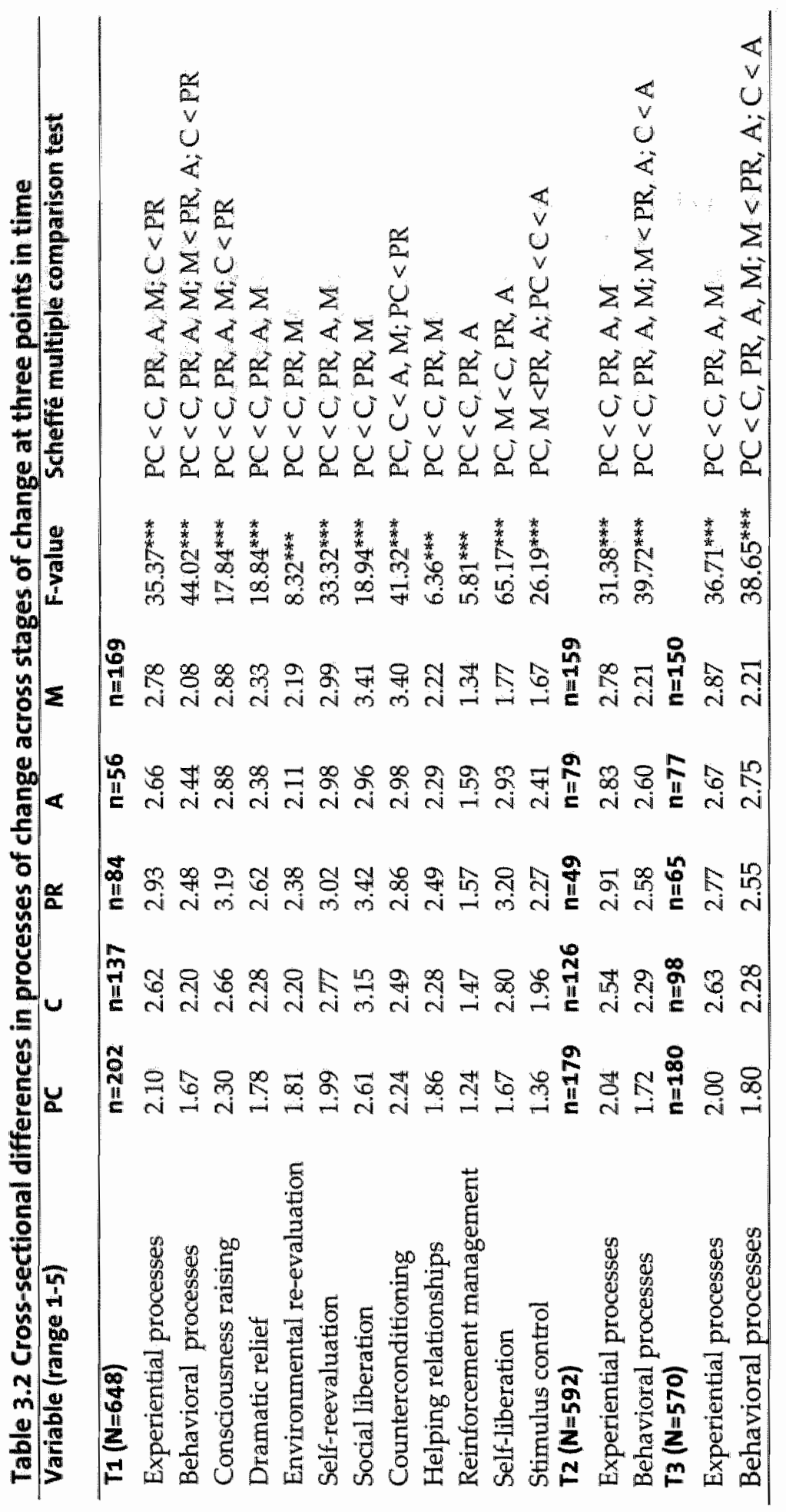




\section{Predictors of stage transitions}

\section{Forward stage transition out of precontemplation}

Of the precontemplators at $\mathrm{T} 1,131(77 \%)$ were still in precontemplation at $\mathrm{T} 2$, while $39(23 \%)$ progressed to a further stage of change. Of the precontemplators at $\mathrm{T} 2,128(83 \%)$ remained in precontemplation at $\mathrm{T} 3$, while $27(17 \%)$ former precontemplators had progressed at T3 (see chapter 2).

More frequent use of experiential processes of change significantly predicted forward stage transition out of precontemplation in both time intervals. Further analyses showed that this stage transition was predicted by more frequent use of consciousness raising, dramatic relief, environmental reevaluation and self-reevaluation, leaving social liberation as a non-significant predictor (Table 3.3). In the T2-T3 interval, in addition to the experiential processes, also more frequent use of behavioral processes predicted forward transition out of precontemplation. Further analyses revealed that more frequent use of reinforcement management, self-liberation, and stimulus control predicted this stage transition.

Table 3.3 Odds ratio (OR) and $95 \%$ confidence intervals (CI) for predictors of forward stage transition from the precontemplation stage for two time intervals

\begin{tabular}{|c|c|c|c|c|}
\hline & \multicolumn{2}{|c|}{ T1-T2 $(N=169)$} & \multicolumn{2}{|c|}{$\mathrm{T}_{2}-\mathrm{T}_{3}(\mathrm{~N}=154)$} \\
\hline & OR & $95 \% \mathrm{Cl}$ & OR & $95 \% \mathrm{Cl}$ \\
\hline Experiential processes & $2.36^{* * 3+}$ & $1.32-4.20$ & $2.52^{* * *}$ & $1.35-4.69$ \\
\hline Behavioral processes & 1.38 & $0.68-2.79$ & $2.56^{* * * 4}$ & $1.33-4.94$ \\
\hline Consciousness raising & $1.65^{*}$ & $\mathbb{1 1 . 0 9 - 2 . 4 5}$ & $1.90^{*}$ & $1.10-3.30$ \\
\hline Dramatic relief & $1.56 \%$ & $11.03-2.37$ & $1.77^{*}$ & $1.05-2.99$ \\
\hline Environmental re-ewalluation & $1.76^{*}$ & $1.14-2.72$ & $1.67^{*}$ & $1.05-2.64$ \\
\hline Self-reevaluation & $1.76^{* 4}$ & $1.20-2.58$ & $2.13^{* * * *}$ & $1.37-3.31$ \\
\hline Social liberation & 1.13 & $0.79 \cdot 1.60$ & 1.44 & $0.92-2.26$ \\
\hline Counterconditioning & 1.18 & $0.82-1.70$ & 1.41 & $0.94-2.12$ \\
\hline Helping relationships & 1.40 & $0.998-1.97$ & 1.23 & $0.80-1.90$ \\
\hline Reinforcement management & 0.94 & $0.43-2.03$ & $1.67^{*}$ & $1.02-2.74$ \\
\hline Self-liberation & 0.87 & $0.56-1.36$ & $2.65^{* * * *}$ & $1.56-4.47$ \\
\hline Stimulus control & 0.95 & $0.52-1.72$ & $1.64^{*}$ & $1.02-2.66$ \\
\hline
\end{tabular}




\section{Forward stage transition out of contemplation}

Of the contemplators at $\mathrm{T} 1,75(65 \%)$ were still in contemplation or regressed to precontemplation at $\mathrm{T} 2$, while $41(35 \%)$ had progressed to a further stage of change. Of the contemplators at T2, $83(76 \%)$ were still in contemplation or regressed to precontemplation at $\mathrm{T} 3$, while $26(24 \%)$ of the former contemplators had progressed at T3 (see chapter 2).

In both time intervals, experiential processes did not predict stage transition out of contemplation. In the T2-T3 interval, a higher frequency of behavioral process use, more specifically counterconditioning and stimulus control, predicted forward stage transition out of contemplation (Table 3.4).

Table 3.4 Odds ratio (OR) and $95 \%$ confidence intervals (CI) for predictors of forward stage transition from the contemplation stage for two time intervals

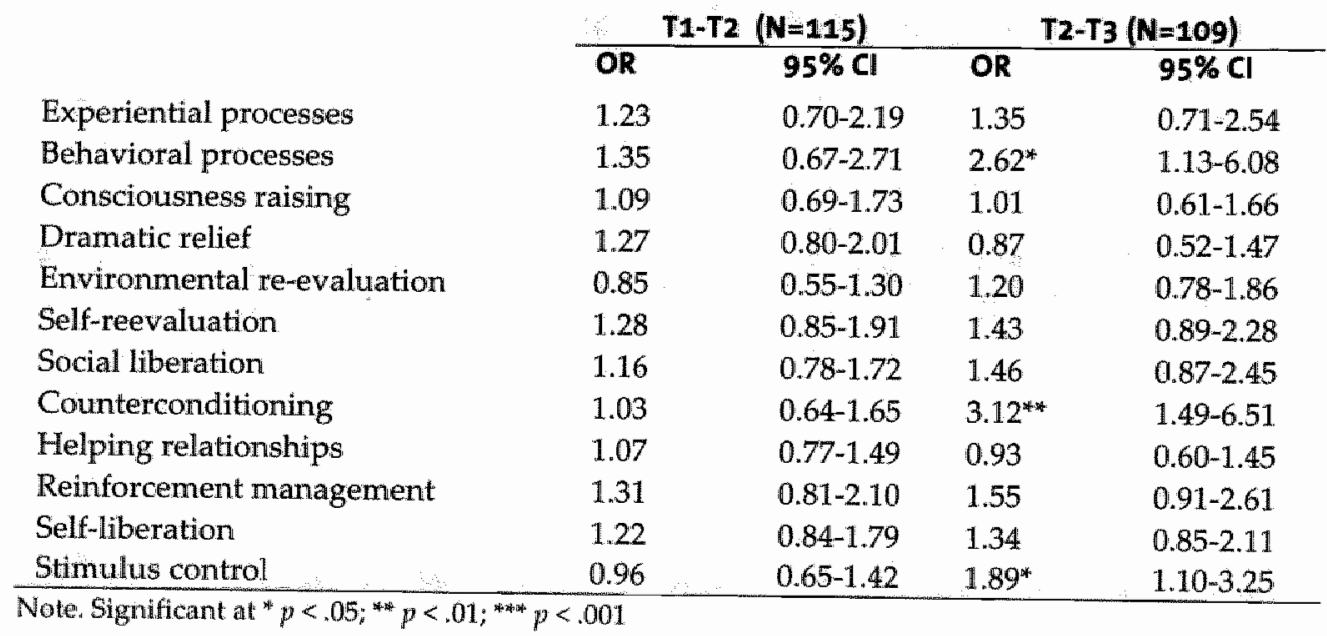

\section{Forward stage transition into the action stage}

Of the respondents in one of the pre-action stages at $\mathrm{T} 1,299(86 \%)$ were still in one of those stages at T2, while $47(14 \%)$ had progressed to the action stage of change. Of the respondents in pre-action stages at $\mathrm{T} 2,275(92 \%)$ remained in one of the pre-action stages at T3, while $25(8 \%)$ had progressed to the action stage at $\mathrm{T} 3$. Between $\mathrm{T} 1$ and $\mathrm{T} 2$, and between $\mathrm{T} 2$ and $\mathrm{T} 3,4$ and 5 respondents progressed from the precontemplation stage to action, respectively.

In both time intervals, stage transition from one of the pre-action stages into the action stage was predicted by more frequent use of experiential as well as behavioral processes. More specifically, frequent use of consciousness raising, 
self-reevaluation, counterconditioning, self-liberation, and stimulus control predicted forward transition into the action stage in both intervals. In the T1-T2 interval, also the dramatic relief process predicted stage transition into action, whereas in the T2-T3 interval the environmental re-evaluation and reinforcement management processes further predicted this transition (Table 3.5).

Table 3.5 Odds ratio (OR) and $95 \%$ confidence intervals $(\mathrm{CI})$ for predictors of forward stage transition into the action stage for two time intervals

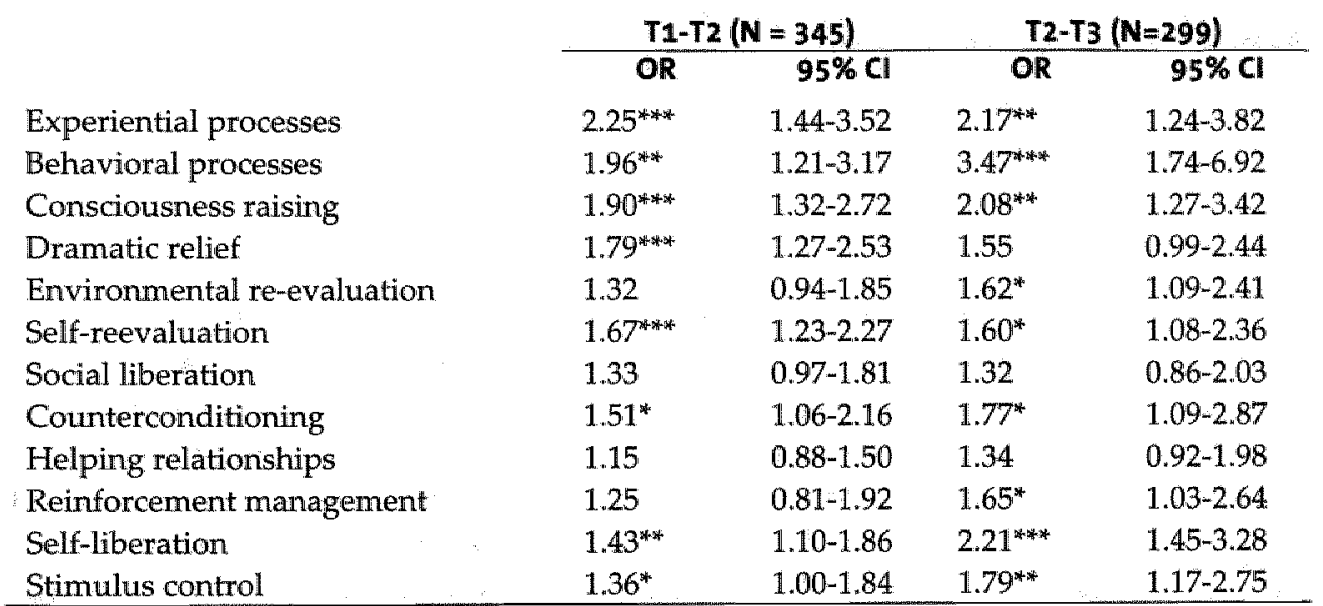

Note. Significant at $* p<.055^{* *} p<.01 ;{ }^{* * *} p<.001$

\section{Predictors of fruit intake}

Forward stage transition out of precontemplation (beta $=.27, p<.001$ ) and contemplation (beta $=.25, p<.01$ ) as well as progress into the action stage (beta = $.46, p<.001$ ) between T1 and T2 predicted fruit intake at T2. Further forward stage transition out of precontemplation (beta $=.27, p<.001$ ) and contemplation (beta $=.51, p<.01)$ as well as progress into the action stage (beta $=.36, p<.001$ ) between $\mathrm{T} 2$ and $\mathrm{T} 3$ predicted fruit intake at $\mathrm{T} 3$. 


\section{Discussion}

The present study showed that increased process use seems associated with more advanced stages of change. First, cross-sectional differences were found for the processes of change, with the largest difference in process use between precontemplation and all other stages consistent with earlier cross-sectional research (Fiontes de Oliveira et al., 2005; Greene et al,, 2004). Experiential as well as behavioral processes increased from precontemplation to action with similar patterns, and with more use of experiential processes than behavioral ones. Second, in the longitudinal analyses, processes of change predicted stage transitions. Both experiential and behavioral processes predicted forward transition out of precontemplation and forward transition into action, while only behavioral processes predicted forward transition out of contemplation.

In line with predictions from the TTM, transition out of precontemplation was indeed predicted by consciousness raising, dramatic relief and environmental re-evaluation (Prochaska \& Velicer, 1997a). Also behavioral processes, such as reinforcement management, self-liberation, and stimulus control seem of importance for this transition, with self-liberation being the strongest predictor of transition out of precontemplation. Notably, the TTM predicts that behavioral processes especially should occur in later stages of change. However, for exercise behavior, similar effects were found in a metaanalysis of cross-sectional studies, with self-liberation as the process with the largest effect size between precontemplation and contemplation (Marshall \& Biddle, 2001). The type of health behavior under study, e.g., a "continuous" behavior that needs to be performed repeatedly, might explain why behavioral processes are already of importance in people who have been allocated to early stages. Defining stages of change for continuous behaviors such as diet or exercise is different than for, for example, smoking or other behaviors for which complete cessation is the goal (Lamb \& Joshi, 2004; Ni Mhurchu et al., 1997; Povey et al,, 1999). Our results showed that all forward transitions predicted fruit intake. Respondents progressing from precontemplation and contemplation may already have made small behavior changes, that did not make them comply to recommended intake levels yet, and are therefore not yet defined to be in action. Nevertheless, such small but not yet sufficient changes in fruit intake may of course very well. require engagement in behavioral processes and strategies to achieve the change.

The TTM postulates that the experiential processes would increase first, followed by an increase in behavioral processes. Our results, however, demonstrated a similar pattern for the experiential and behavioral processes across the stages of change. In two meta-analyses of cross-sectional studies, 
similar results for dietary change and exercise were found (Marshall \& Biddle, 2001; Rosen, 2000). It has been argued that the two-factor higher order structure may not be distinguishable for these behaviors and that the processes might as well reflect one factor (Marshall \& Biddle, 2001; Rosen, 2000). From a different point of view, this finding might imply that treatments matched to individuals in early stages should not solely focus on cognitive change, but opportunities may already exist for the incorporation of small behavioral changes or at least changes that prepare for the desired behavior. Rosen (2000) found large effects for stimulus control in dietary change between stages, and our results replicate his findings. Thus, creating a stimulating environment by adding cues to eat fruits (stimulus control) could already be relevant for individuals in early stages. As a result, when people start consuming adequate amounts of fruit, the focus should not exclusively be on behavioral strategies, but, for example consciousness raising activities like looking for recipes and reading other information about fruits may remain important issues, even in action.

According to the TTM, the self-reevaluation process of change should predict forward transition out of the contemplation stage. Our results showed that self-reevaluation was relevant for transition out of precontemplation, but not for transition out of the contemplation stage for fruit intake. Other people have suggested that self-monitoring and awareness of one's personal performance, which may be close to self-reevaluation, is an important prerequisite for motivation (Lechner et al., 1998; Ronda et al., 2001). Remarkably, none of the experiential processes, but only behavioral processes (counterconditioning and stimulus control) predicted the transition out of contemplation.

Overall, eight out of ten processes were relevant for one of the stage transitions for fruit intake, with the exception of social liberation and helping relationships. Past research also showed that social liberation is difficult to place along the stages of change continuum and this process might therefore not be predictive of stage transitions (Redding et al., 1999). The fact that having helping relationships was not predictive of transitions may, on the one hand, be attributed. to the type of health-related behavior under study. It may be that for fruit intake the encouragement of others (in contrast to e.g., smoking cessation) is not very important. On the other hand, Rosen (2000) also found weak effects for having helping relationships in dietary change, and explains the lack of effect by the idea that use of social support may depend on the availability of social support, which may be unrelated to stage of change.

Even though our results seem to support the importance of the Transtheoretical processes of change for the induction of progression through the stages of change for fruit intake, some comments on the concept of processes of change must be acknowledged. First, processes of change were not stage-specific, since similar processes of change were relevant for more than one transition in 
stages of change. This conflicts with an important suggested implication of stages of change: stage-targeting of nutrition education. Second, according to the TTM, processes of change should be assessed retrospectively. However, recalling the use of processes of change may not be accurate. Some people may not be aware of having applied particular processes in the past, while people who are more involved in the issue of fruit intake (e.g, respondents who progress to action) might be more able to recall the applied processes of change. Third, to justify the term 'processes of change', effects of actual use of these so-called processes should preferably be tested in experimental research. The questionnaire items regularly used to assess the experiential processes do not assess actual use, but might better be described as beliefs. The processes may therefore be similar to, for example, outcome expectancies from Bandura's Social Cognitive Theory (Bandura, 1986).

Furthermore, some limitations of our study must be considered. In the present study a processes of change scale with only one item per process was used, based on measurement instruments developed for other behaviors (McKenna \& Francis, 2003; Prochaska et al., 1988; Rossi et al., 1994). The aggregated experiential and behavioral processes measures (with five items each), operationalized as outlined by the TTM, were the point of departure in the analyses instead of the separate single item measures for separate processes. These aggregate measures showed moderate to good internal consistencies and good test-retest reliabilities. However, further validation of the scale is warranted. Further, the existing Internet-panel sampling frame may not have been representative of the Dutch population. Compared to the general Dutch population, higher educated people and respondents younger than 30 were overrepresented; lower educated people and respondents over 55 years old were underrepresented in the Internet-panel sample; and the prevalence of eating less of than two servings of fruit each day was lower in the Internet-panel than in the Dutch population (Statistics Netherlands, 2003; Van Oers, 2002). On the other hand, the Internet may provide a way to include populations that are normally difficult to access, such as precontemplators (Nosek, Banaji, \& Greenwald, 2002). The use of an Internet-panel also provided the opportunity to have repeated measurements within short time intervals. Several longitudinal studies showed non-significant relations between processes of change and transitions in stage of change (Herzog et al., 1999; Nigg, 2001; Segan et al., 2004), which may be due to the larger time intervals between measurements in those studies. As Nigg (2001) pointed out, the processes of change are thought of as dynamic change variables, and increasing time intervals between assessments would dampen direct relations. 


\section{Implications for research and practice}

Despite the limitations, the present study provides indications that the Transtheoretical processes of change predict stage movements for fruit intake, but that the pattern of relevant processes for fruit intake is not as straightforward as outlined by the TTM. Though the lack of stage-specificity will make stagetargeting difficult, the results from the present study do indicate that it is indeed important to include the processes of change in health promoting interventions aimed at stage progression for increasing fruit intake. Further research in a more representative sample is necessary to gain insight into how engagement in processes of change can be effectively increased. 


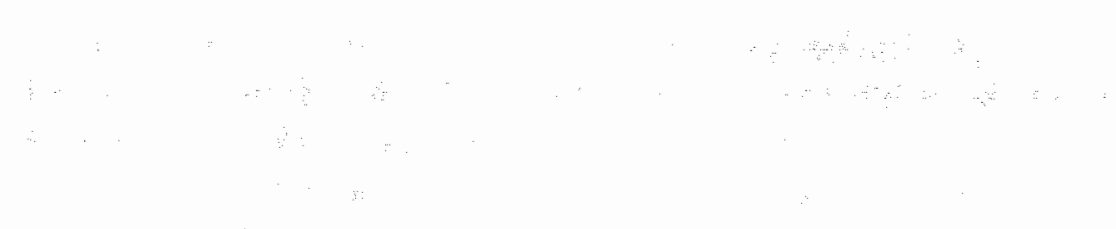




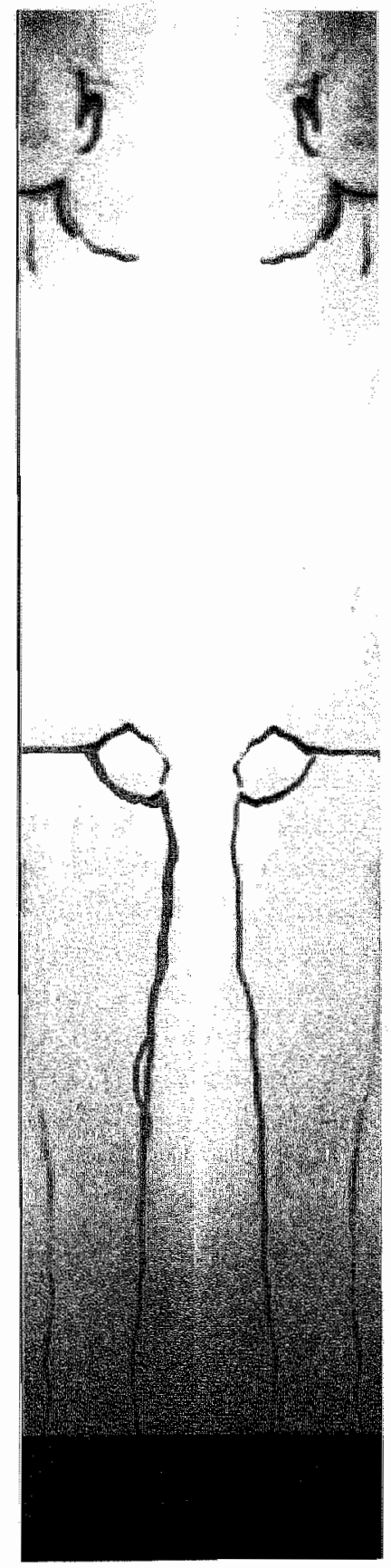

\section{Part 2 Staging health behavior change}


$+49$

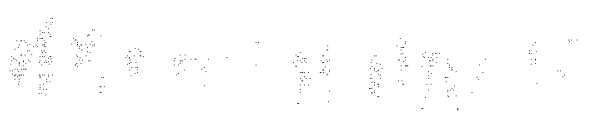

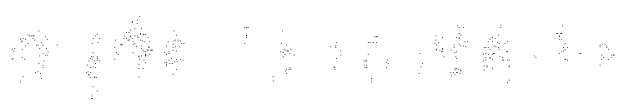

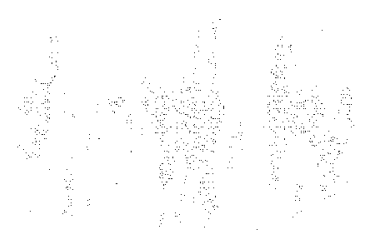




\section{Chapter 4 \\ Stages of change in fruit intake: a longitudinal examination of stability, stage transitions and transition profiles}

\section{Abstract}

The present study examined stability and transitions in Transtheoretical stages of change for fruit intake in an adult cohort of 735 individuals not exposed to a planned intervention. Six assessments took place within a four-month period with varying time intervals using electronic questionnaires, measuring fruit intake, stage of change and demographic characteristics. Precontemplation and maintenance were the most stable stages of change, while preparation was the least stable. Longer time intervals showed lower stability, but frequent stage transitions also occurred in shorter time intervals. Seven transition profiles were distinguished. Sequences within transition profiles mostly showed stage transitions to adjacent stages, independent of the interval duration. Differences in fruit intake, age, level of education and ethnicity were found between different transition profiles. Stability rates and most transition sequences largely support the Transtheoretical Model. Results indicating potential deviations from the model, e.g., short-term stage instability and stage transition profiles not in accordance with the Transtheoretical Model are discussed.

Published as: De Vet, E., De Nooijer, I., De Vries, N. K., \& Brug, J. (2005). Stages of change in fruit intake: a longitudinal examination of stability, stage transitions and transition profiles. Psychology and Health, 20,415-428 


\section{Introduction}

Stage models of behavior change are widely used in health promotion, health psychology, as well as in non-health domains like marketing (O'Brien, 1971; Schwarzer, 1992b; Weinstein \& Sandman, 1992). The most widely used stage model in health promotion is the Transtheoretical Model (TTM; Prochaska \& DiClemente, 1983; Prochaska \& Velicer, 1997a), which distinguishes five so-called stages of change. In the first stage, precontemplation, people express not to intend changing their (unhealthy) behavior within the next six months. The TTM uses a six-month time frame because it assumes that this is about as far in the future as most people plan a specific behavior change (Prochaska et al., 1994; Velicer et al., 1995). In the second stage, contemplation, people do intend to change their behavior in the next six months, but not within 30 days. People in preparation intend to change within 30 days. When someone has changed from unhealthy behavior to healthy behavior in the past six months, the action stage is reached, and people are in the maintenance stage when they have maintained this behavior change for more than six months.

Using stage models can serve several purposes. First, stage models provide the opportunity to identify specific target group segments within a population and to match interventions to stage of change (e.g., Aveyard, Griffin, Lawrence, \& Cheng, 2003; Clark, Nigg, Greene, Riebe, \& Saunders, 2002; Prochaska, Velicer, Fava, Rossi, \& Tsoh, 2001). Second, changes in stages of change, i.e., stage transitions, can and have been used as an intermediate impact indicator in the evaluation of behavior change interventions (e.g., Brug \& Van Assema, 2000; Gielen et al., 2001). Finally, stage models are used to gain insight into the process of health behavior change (e.g., Dijkstra et al., 2003; McKenna \& Francis, 2003; Rosen, 2000; Velicer et al., 1999). The present study contributes to the examination of the process of health behavior change by describing stage transition patterns longitudinally for fruit intake in an adult cohort not exposed to a planned intervention. In order to use stages of change for stage-matched interventions or to evaluate behavior change interventions, stages of change need to be stable to a certain extent. Nevertheless, self-change in the absence of planned interventions is possible, because people are exposed to numerous health behavior change messages every day via the media as well as through people in their social environment. Thus, stage transitions are expected to occur, even when people are not exposed to a formal planned intervention. To date, no guidelines are available on the amount of stage transitions that are acceptable for the development and evaluation of (stage-matched) behavior change interventions, so more insight into stability and changeability of stages of change is needed.

So far, only a few studies have examined changes in stage of change over time (Courneya et al., 2001; De Nooijer, Van Assema, De Vet, \& Brug, 2005; 
Norman, Velicer, Fava, \& Prochaska, 1998; Schumann, Meyer, Rumpt, Hapke, \& John, 2002). For example, Schumann and colleagues (2002) examined changes in stages of change to quit smoking between two time points using a six-month time interval. They concluded that without an intervention, there is no substantial smoking behavior change toward abstinence. De Nooijer et al. (2005) examined stage stability for nutrition behaviors, i.e., fat, vegetable and fruit intake. Their results indicated frequent stage transitions in absence of planned interventions.

An interesting approach to study stage transitions is to describe patterns of stage transitions (Norman et al., 1998; Prochaska et al., 1991). Prochaska et al. (1991) constructed a developmental profile of the entire change process from precontemplation to maintenance for smoking cessation, distinguishing 14 patterns of change. They focused on successful changers, and therefore, unstable patterns of change were not considered. Norman and colleagues (Norman et al., 1998) studied stable as well as unstable patterns of change in smoking cessation in a representative sample and identified four stage transition patterns: stable, progress, regress and vacillating. People in the stable typology were in the same stage at each of the five stage assessments. People in progress showed forward stage transition between the first and last assessment. The regress typology represented people who showed stage regression between baseline and last follow-up. Vacillating was characterized by stability between baseline and followup, with stage transitions in-between. The typologies introduced by Norman and colleagues (1998) provide an initial framework for investigating stage stability and stage transitions over time. However, their four-category typology may not be sufficient to describe all relevant patterns of stage stability and stage transition. For example, people who show progress between baseline and last follow-up, do not necessarily show steady progress over time.

The available stage transition studies all used six-month time intervals. In such a relatively long period of time, it is, for example, to be expected that people are exposed to various relevant health messages that may induce stage transitions. Therefore, studies describing stage transition patterns within shorter time intervals are also needed. Further, when patterns of change are examined, it is important to take into account the path or route people follow within that pattern, the so-called sequence. For example, when in a progress typology, a person proceeding from precontemplation to action differs from someone who progresses from precontemplation to contemplation in the same period of time. Finally, more insight is needed into qualitative and distinctive differences between persons in different stage transition patterns, for example, in demographic factors.

The present study aimed (1) to examine stage stability and naturally occurring stage transitions in a four-month period with five time intervals varying from several days to several weeks, (2) to describe distinctive patterns of stage transitions and stage stability, and the specific sequences within these 
patterns of stage transitions, and (3) to investigate differences between people in these patterns according to sex, age, level of education, ethnicity and fruit intake.

In the present study stages of change related to fruit intake were measured, since ample consumption of fruit is considered to contribute to the prevention of cardiovascullar disease (Trichopoulou \& Lagiou, 1997), certain cancers (Key et al., 2002; Paolini et al., 2003), as well as obesity (Lanza et al., 2001; Pesa \& Turner, 2001), while approximately $70 \%$ of the Dutch population eat less than the recommended minimum amount of two servings (250 grams) of fruit per day (Dutch Nutrition Centre, 1998; Van Oers, 2002). Furthermore, fruit intake is a valuable behavior to relate to stages of change, since fruit intake is a repetitive behavior, i.e., the recommended health behavior needs to be performed every day. It may therefore be well suited to map patterns of stage transitions and stage stability.

\section{Method}

\section{Participants and procedures}

Potential participants were identified from a random sample of 1,500 people aged 18 years and older from a Dutch Internet panel. At the time of the study, the size of the entire panel was about 8,165 persons. People were invited by an email letter to participate in a longitudinal cohort study. Respondents could indicate their willingness to participate in the entire study by completing the first electronic questionnaire (T0). Completed questionnaires were returned by 929 respondents (response rate $=62 \%$ ). Thirteen respondents were excluded from the study because stage of change or fruit consumption could not be calculated $(n=916$, response rate $=61 \%$ ). Of the 916 respondents, for each stage of change at baseline, $20 \%$ was selected randomly to participate in a different study. The remaining 735 respondents comprised the present study's cohort. Mean age was 37.5 ( $\mathrm{SD}=14.1$ ) years, and women and men were equally represented (51\% vs. $49 \%$ ). Of the respondents, $17 \%, 48 \%$ and $36 \%$ reported a low medium and high level of education, respectively. Most respondents were of Dutch origin (90\%). Mean fruit intake was $260(\mathrm{SD}=163$ ) grams per day, while $57 \%$ of the respondents ate less fruit than the recommended minimum intake level of 250 grams a day. At baseline (T0), $242(33 \%)$ respondents were in precontemplation, $157(21 \%)$ in contemplation, $104(14 \%)$ in preparation, $49(7 \%)$ in action and $183(25 \%)$ in maintenance. For the six assessments, the proportion of respondents eating according to recommendations ranged from $63 \%$ to $73 \%$ for the action stage and from $78 \%$ to $85 \%$ for the maintenance stage. The proportion of respondents not 
eating according to recommendations ranged from $79 \%$ to $85 \%$ for precontemplation, from $71 \%$ to $83 \%$ for contemplation and from $48 \%$ to $80 \%$ for preparation. Demographic information for each baseline stage of change is described in Table 4.1.

Table 4.1 Demographic information for each baseline stage of change

\begin{tabular}{|c|c|c|c|c|c|}
\hline & $\begin{array}{l}P^{2} \\
(\mathrm{n}=242 ; \\
32.9 \%)\end{array}$ & $\begin{array}{l}C \\
(n=157 \\
21.4 \%)\end{array}$ & $\begin{array}{l}P R \\
(n=104 ; \\
14.1 \%)\end{array}$ & $\begin{array}{l}A \\
(n=49 \\
6.7 \%)\end{array}$ & $\begin{array}{l}M \\
(n=183 ; \\
24.9 \%)\end{array}$ \\
\hline Mean age (SD) & $\begin{array}{l}37.5 \\
(13.7)\end{array}$ & $\begin{array}{l}34.0 \\
(12.8)\end{array}$ & $\begin{array}{l}34.9 \\
(13.2)\end{array}$ & $\begin{array}{l}33.7 \\
(12.1)\end{array}$ & $\begin{array}{l}43.1 \\
(15.0)\end{array}$ \\
\hline \multicolumn{6}{|l|}{ Sex } \\
\hline Female & $44.2 \%$ & $57.3 \%$ & $59.6 \%$ & $61.2 \%$ & $44.8 \%$ \\
\hline Male & $55.8 \%$ & $42.7 \%$ & $40.4 \%$ & $38.8 \%$ & $55.2 \%$ \\
\hline \multicolumn{6}{|l|}{ Level of education } \\
\hline Low level & $19.8 \%$ & $14.6 \%$ & $11.5 \%$ & $16.7 \%$ & $16.6 \%$ \\
\hline Medium level & $45.5 \%$ & $52.9 \%$ & $46.2 \%$ & $56.3 \%$ & $44.2 \%$ \\
\hline High level & $34.7 \%$ & $32.5 \%$ & $42.3 \%$ & $27.1 \%$ & $39.2 \%$ \\
\hline \multicolumn{6}{|l|}{ Ethnicity } \\
\hline Dutch origin & $92.1 \%$ & $89.8 \%$ & $86.5 \%$ & $89.8 \%$ & $89.6 \%$ \\
\hline Foreign origin & $7.9 \%$ & $10.2 \%$ & $13.5 \%$ & $10.2 \%$ & $10.4 \%$ \\
\hline Mean fruit intake grams/day & 186 & 208 & 241 & 338 & 391 \\
\hline$(S D)$ & (141) & (97) & $(117)$ & $(167)$ & $(168)$ \\
\hline $\begin{array}{l}\text { Eating according to } \\
\text { recommendations }\end{array}$ & $21.3 \%$ & $29.3 \%$ & $38.5 \%$ & $62.5 \%$ & $81.9 \%$ \\
\hline
\end{tabular}

Note, $\mathrm{PC}=$ precontemplation, $\mathrm{C}=$ contemplation, $\mathrm{PR}=$ preparation, $\mathrm{A}=$ action, $\mathrm{M}=$ maintenance.

After baseline measurement (T0), five follow up measurements (T1-T5) were conducted with a $3,14,21,29$, and 53-days time interval, using the same questionnaire every time (Table 4.2). Different time intervals were used to study whether smaller time intervals would reveal higher stability. Also, in stage-based interventions the delivery of the interventions commonly takes place some time after stage assessment. To explore whether delivery of stage-based interventions can still be accurate days after stage assessment, small time intervals were used (e.g., 3, 14 and 21 days). The 29-day interval reflects the period in which preparators intend to change behavior and can be used to study whether progress actually occurs within one month. For the planning of each of the assessments bank and school holidays were taken into account and questionnaires were only sent on weekdays, since respondents needed computers to complete questionnaires and most did so during working hours. 
Table 4.2 study time frame, number of respondents and response rates

\begin{tabular}{llll}
\hline Assessment & $\begin{array}{l}\text { Days after previous } \\
\text { assessment }\end{array}$ & Number of respondents & Response rate \\
\hline T0 & 0 & 735 & - \\
T1 & 14 & 648 & $88 \%$ \\
T2 & 21 & 610 & $83 \%$ \\
T3 & 29 & 495 & $67 \%$ \\
T4 & 3 & 595 & $81 \%$ \\
T5 & 53 & 570 & $78 \%$ \\
\hline
\end{tabular}

In total, $376(51 \%)$ respondents completed all questionnaires. Eighty-six percent of the respondents completed four or more questionnaires. Respondents who did not complete all six questionnaires were significantly younger $(F(1,735)$ $=28.77$; Cohen's $d=0.40 ; p<.001)$ and are more often of foreign origin $\left(\chi^{2}(1, N=\right.$ $\left.735)=7.83, r_{w}=.10 ; p<.01\right)$ than those who completed all six questionnaires. No differences between completers and non-completers were found with respect to sex, level of education, stage of change or fruit intake.

Respondents used a personal login code to open and complete the questionnaires. Answers could not be saved and the questionnaire could not be completed more than once. Respondents did not know in advance when questionnaires were sent and each of the questionnaires had a different web address. Questionnaires had to be completed in a single session within one week after the emailed completion request, except for the T3 questionnaire. To make sure that $\mathrm{T} 3$ and 'T4 were not interfering, the $\mathrm{T} 3$ questionnaire had to be completed within one and a half days. Respondents' answers were automatically entered into a data file, and after the deadline had passed by, the respondents could not enter the questionnaire anymore. Table 4.2 shows the study time frame, number of respondents and response rates for each of the follow-up measurements.

\section{Measures}

Stage of change was assessed consistent with Nigg and colleagues (1999) and Laforge et al. (1999) and in line with the definitions determined by the TTM, by asking respondents to select the most suitable statement out of five statements each representing a stage of change: "I do not eat at least two servings of fruit every day, and I do not intend to change this within the next six months" (precontemplation), "I do not eat at least two servings of fruit every day, but 1 intend to change this within the next six months" (contemplation), "I do not eat at 
least two servings of fruit every day, but $I$ intend to change this within the next month" (preparation), "I eat at least two servings of fruit every day, and I've started doing this in the last six months" (action), and "I eat at least two servings of fruit every day, and I have done so for more than six months" (maintenance).

Fruit intake was assessed with a 14-item food frequency questionnaire (FFQ), validated as compared to a 7 -day dietary record $(r=.51 ; p<.001$; Van Assema et al., 2002) and biomarkers for fruit consumption levels, i.e., blood levels of total carotenoids $(r=.34 ; p<.001)$, vitamin $C(r=.27 ; p<.001)$ and betacryptoxanthin $(r=.42 ; p<.001$; Bogers et al., 2004). Respondents were asked about consumption during the previous month. The FFQ included questions about frequency of consumption (from "never/less than one time per month" to "7 days a week") and number of servings (pieces or small bowls [for small fruit such as berries] per day that a certain fruit (juice) was consumed). Separate questions were included for the most common fruits in the Netherlands (citrus fruit, such as oranges and grapefruit; apples or pears; bananas), for an important seasonal fruit (tangerines), for freshly squeezed or other unsweetened fruit juice, and for "other fruit including preserved fruit". To calculate total fruit consumption, serving sizes were first transformed to grams based on standard mean weights of the fruits. Subsequently, for each fruit category frequency of consumption was multiplied with serving size and the results were added up and divided by seven to obtain a fruit intake score in grams per day.

Demographic variables included sex, age, education and ethnicity, and were only assessed at baseline. Education was measured by asking the respondents about the highest level of education they had completed. Respondents who indicated that they had no education, primary school, secondary school, and lowest level of high school or lower vocational training were classified as having a low level of education. Respondents with a medium level of education had completed intermediate or high level high school, or medium level vocational training. Respondents who had completed higher vocational training, college or university education had a high level of education. Ethnicity of respondents was measured by asking respondents the country of birth for both respondents ${ }^{\prime}$ parents. If one or both parents had been born outside the Netherlands, respondents were classified as "of foreign origin". If both parents had been born in the Netherlands, respondents were classified as "of Dutch origin". 


\section{Results}

\section{Stage stability and stage movements}

Stability between two time points for each time interval was computed for respondents who completed both relevant measurements, by means of stability rates per stage of change (percentages of respondents being in the same stages of change at both measurements) and Cohen's Kappa. Generally, a Kappa > .75 is considered excellent agreement, a Kappa between .40 and .75 as fair to good and a Kappa <.40 as poor (Fleiss, 1981).

Table 4.3 Stability rates and Cohen's kappa between consecutive time points

\begin{tabular}{|c|c|c|c|c|c|}
\hline & $\begin{array}{c}\text { To-T1 } \\
14 \text { days }\end{array}$ & $\begin{array}{c}T 1-T 2 \\
21 \text { days }\end{array}$ & $\begin{array}{c}\text { T2-T3 } \\
29 \text { days } \\
\end{array}$ & $\begin{array}{l}T 3-T 4 \\
3 \text { days } \\
\end{array}$ & $\begin{array}{c}\text { T4-T5 } \\
53 \text { days }\end{array}$ \\
\hline PC stable & $74.3 \%$ & $80.1 \%$ & $80.8 \%$ & $88.2 \%$ & $82.6 \%$ \\
\hline C stable & $51.8 \%$ & $58.5 \%$ & $62.9 \%$ & $77.9 \%$ & $57.8 \%$ \\
\hline PR stable & $45.1 \%$ & $38.7 \%$ & $36.4 \%$ & $50.0 \%$ & $54.5 \%$ \\
\hline A stable & $45 \%$ & $60.4 \%$ & $72.4 \%$ & $76.4 \%$ & $59.4 \%$ \\
\hline M stable & $87.5 \%$ & $87.4 \%$ & $86.4 \%$ & $90.2 \%$ & $85.8 \%$ \\
\hline Kappa & $.567^{* * * *}$ & $614^{* * *}$ & $.655^{* * *}$ & $.761^{* * *}$ & $.647^{* * * *}$ \\
\hline
\end{tabular}

Table 4.3 shows that the precontemplation (stability rate ranged from $74 \%$ to $83 \%$ ) and maintenance (stability rate ranged from $86 \%$ to $90 \%$ ) stages were the most stable, while preparation (stability rate ranged from $36 \%$ to $55 \%$ ) was the least stable stage of change. It was expected that stability rates would be highest for T3T4, followed by T0-T1, T1-T2, T2-T3 and lowest for T4-T5. Only the maintenance stage follows this exact outline. Nevertheless, most stages of change show highest stability rates on the shortest time interval. (T3-T4), with exception of the preparation stage. On the contrary, for the preparation stage, the highest stability rate is established in the longest time interval (T4-T5). Kappa's between consecutive follow-ups varied from $.56(p<.001)$ between T0 and T1 and .76 ( $p<$ .001 ) between T3 and T4 (see Table 4.3). The latter was the only Kappa that indicated excellent agreement according to Fleiss (1981). None of the time intervals revealed a poor agreement in classification in stages of change. Table 4.4 depicts the occurring stage transitions between $\mathrm{T} 2$ and $\mathrm{T} 3$ as an example. This pattern was largely similar in all other time intervals. Respondents in preparation had a similar chance to regress or progress in each time interval. 
Respondents in precontemplation were most likely to progress to contemplation, again for all time intervals. Respondents who changed from contemplation or action moved to every possible other stage.

\section{Table 4.4 Stage transitions between two consecutive follow-ups}

\begin{tabular}{|c|c|c|c|c|c|c|}
\hline \multirow[t]{11}{*}{$\begin{array}{l}\mathrm{T2} \\
\% \text { of } \mathrm{T} 2\end{array}$} & T3 & PC & c & $\mathrm{Pr}$ & A & $M$ \\
\hline & PC & 122 & 16 & 1 & 3 & 9 \\
\hline & & $80.8 \%$ & $10.6 \%$ & $0.7 \%$ & $2.0 \%$ & $6.0 \%$ \\
\hline & $c$ & 10 & 56 & 11 & 12 & - \\
\hline & & $11.2 \%$ & $62.9 \%$ & $12.4 \%$ & $13.5 \%$ & \\
\hline & $\mathrm{Pr}$ & - & 12 & 16 & 14 & 2 \\
\hline & $P r$ & & $27.3 \%$ & $36.4 \%$ & $31.8 \%$ & $4.5 \%$ \\
\hline & $A$ & 1 & 5 & 5 & 42 & 5 \\
\hline & A & $1.7 \%$ & $8.6 \%$ & $8.6 \%$ & $72.4 \%$ & $8.6 \%$ \\
\hline & & 6 & - & 2 & 8 & 102 \\
\hline & $m$ & $5.1 \%$ & & $1.7 \%$ & $6.8 \%$ & $86.4 \%$ \\
\hline
\end{tabular}

\section{Transition profiles and sequences}

For the description of the underlying patterns of stage of change transitions, transition profiles over all six measurements (TO-T5) were analyzed, resulting in seven transition profiles: Stable, Progress, Regress, Progress then Regress, Regress then Progress, Multiple Change and Impossible (Figure 4.1). Respondents who were in the same stage at all six measurements were regarded as Stable. Respondents who showed forward stage transition betwreen T0 and T5 with no backward stage transitions on any of the measurements in-between were defined as being in Progress. Similarly, respondents who showed backward stage transition between T0 and T5 and no forward stage transition on any of the measurements in-between were in Regress. Respondents who first showed forward stage transition(s) followed by backward transition(s) were in Progress then Regress, and the opposite pattern was defined as Regress then Progress. Respondents in the Multiple Change profile showed a vacillating pattern of stage of change, characterized by stage transitions in several directions during the fourmonth period. Respondents were classified in the Impossible profile when they reported being in precontemplation, contemplation or preparation at one measurement and at the next measurement regard themselves as maintainers (which is impossible according to the time restraints prescribed by the TTM). Transition profiles were only calculated for respondents who completed all six questionnaires $(n=376)$. 

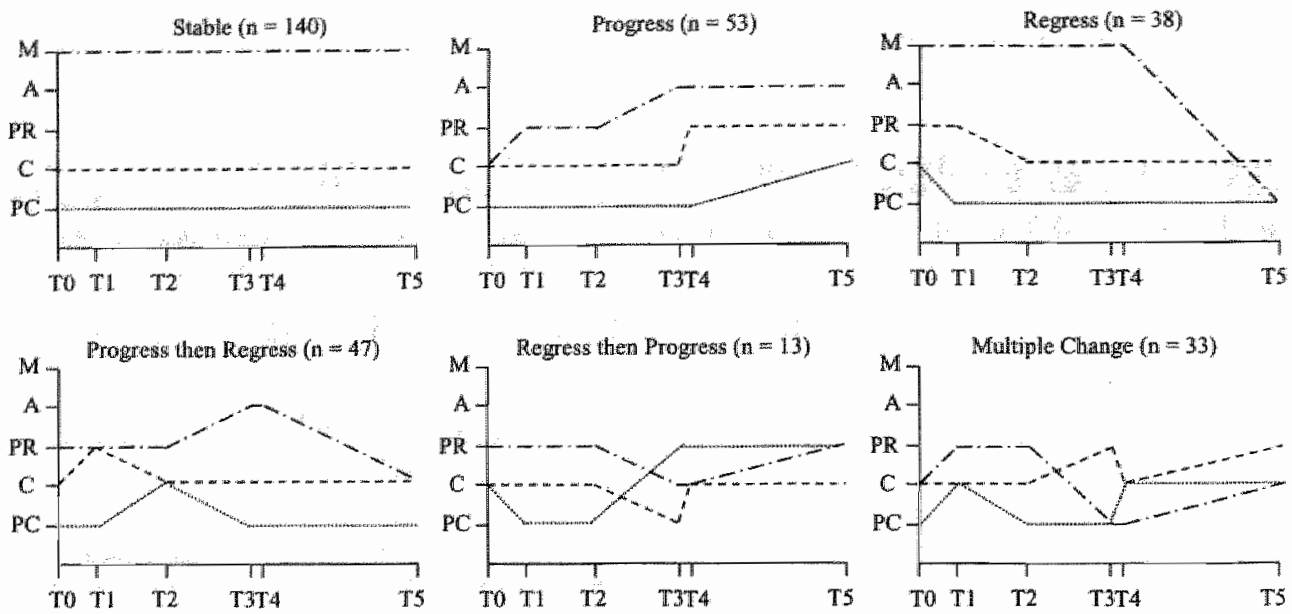

Figure 4.1 Transition profiles with three example sequences in each profile

The Stable transition profile was the most prevalent $(n=140,37 \%)$ and the Regress then Progress profile the least prevalent $(n=13,4 \%$ ) (Table 4.5). Respondents in precontemplation $(n=57$ out of 140$)$ and maintenance $(n=65$ out of 140) were most likely to be Stable. Respondents in contemplation at baseline were most likely to be in Progress then Regress ( $n=16$ out of 47 ), respondents in preparation at baseline were most likely to show Regress $(n=13$ out of 38 ), and respondents in action at baseline were most likely to show the Multiple Change $(n=5$ out of 33$)$ and Impossible profiles $(n=5$ out of 52$)$.

Further, to describe specific sequences in stages of change within each of the transition profiles, we analyzed stage of change at each of the assessments for each of the 376 respondents. There are 15,625 possible stages of change sequences with measurements at six points in time $\left(5^{6}\right)$, but only 170 sequences were represented in our data. The most prevalent sequences in the Stable profile concern respondents who were in maintenance $(n=65$ out of 140 ) or precontemplation $(n=57$ out of 140$)$ at all six measurements. Analyses of the sequences within the unstable profiles revealed that (1) most stage transitions involved a forward or backward stage transition to an adjacent stage somewhere in time, and (2) that also unstable profiles showed more or less stability, with a stage of change that is at only one point in time deviant from stages of change at other assessments. In the subsection below, we depict the most prevalent sequences for each of the transition profiles. In the Progress profile, the PC-PCPC-PC-PC-C and the PR-PR-A-A-A-A sequence both occur four times ( $n=4$ out of 53). Respondents in Regress most frequently showed C-PC-PC-PC-PC-PC ( $n=$ 
8 out of 38) and PR-C-C-C-C-C $(n=5$ out of 38). The PC-PC-C-PC-PC-PC ( $n=5$ out 47), PC-C-PC-PC-PC-PC ( $\mathrm{n}=4$ out of 47$), C-P R-C-C-C-C(n=4$ out of 47$)$ sequence occur most frequent in the Progress then Regress profile. In the Impossible profile respondents most frequent show the M-M-PC-M-M-M ( $n=6$ out of 52) sequence. In the Multiple Change profile and the Regress then Progress profile all sequences only occur once (stage transition pictures for other time intervals and more information on sequences can be obtained from the authors)

Table 4.5 Prevalence of transition profiles by stage at baseline

\begin{tabular}{|c|c|c|c|c|c|c|c|}
\hline \multirow{2}{*}{$\begin{array}{l}\text { Stage To } \\
\% \text { stage } \\
\text { To) }\end{array}$} & \multirow[b]{2}{*}{$\begin{array}{l}\text { Stablle } \\
(n=140)\end{array}$} & \multicolumn{4}{|c|}{ Transition profile } & \\
\hline & & $\begin{array}{l}\text { Progress } \\
(n=53)\end{array}$ & $\begin{array}{l}\text { Regress } \\
(n=38)\end{array}$ & $\begin{array}{l}\text { Progress } \\
\text { then } \\
\text { Regress } \\
(n=47)\end{array}$ & $\begin{array}{l}\text { Regress } \\
\text { then } \\
\text { Progress } \\
(n=13)\end{array}$ & $\begin{array}{l}\text { Impossible } \\
(n=52)\end{array}$ & $\begin{array}{l}\text { Multiple } \\
\text { Change } \\
(n=33)\end{array}$ \\
\hline \multirow[t]{2}{*}{$\mathrm{PC}$} & 57 & 28 & - & 24 & - & 7 & 10 \\
\hline & $45.2 \%$ & $22.2 \%$ & & $19.0 \%$ & & $5.6 \%$ & $7.9 \%$ \\
\hline \multirow[t]{2}{*}{ C } & 11 & 13 & 11 & 16 & 2 & 8 & 11 \\
\hline & $15.3 \%$ & $18.1 \%$ & $15.3 \%$ & $22.2 \%$ & $2.8 \%$ & $11.1 \%$ & $15.3 \%$ \\
\hline \multirow[t]{2}{*}{ PR } & 4 & 8 & 13 & 6 & 8 & 5 & 6 \\
\hline & $8.0 \%$ & $16.0 \%$ & $26.0 \%$ & $12.0 \%$ & $16.0 \%$ & $10.0 \%$ & $12.0 \%$ \\
\hline \multirow[t]{2}{*}{ A } & 3 & 4 & 3 & 1 & 3 & 5 & 5 \\
\hline & $12.5 \%$ & $16.7 \%$ & $12.5 \%$ & $4.2 \%$ & $12.5 \%$ & $20.8 \%$ & $20.8 \%$ \\
\hline \multirow[t]{2}{*}{ M } & 65 & - & 11 & - & - & 27 & 1 \\
\hline & $62.5 \%$ & & $10.6 \%$ & & & $26.0 \%$ & $1.0 \%$ \\
\hline
\end{tabular}

\section{Differences between transition profiles}

\section{Differences in fruit intake}

MANOVA repeated measures revealed no significant main effect of time on fruit intake over the six assessments $(F(4,350)=1.40, n s)$, but a significant time $x$ transition profile interaction effect was found, indicating that the change in fruit intake depended on the transition profile $\left(\mathrm{F}(27,350)=3.21 ; \eta^{2}=.05 ; p<.001\right)$. Further analyses of the separate profiles showed that fruit intake changed significantly during the period of research for the Stable $\left(\mathrm{F}(4,127)=2.75 ; \eta^{2}=.02\right.$; $p<.05)$, the Progress $\left(\mathrm{F}(3,50)=6.14 ; \eta^{2}=.11 ; p<.001\right)$, and the Regress profile ( $\mathrm{F}$ $\left.(4,36)=5.64 ; \eta^{2}=.14 ; p<.001\right)$. For these three profiles, repeated contrast analyses were conducted to compare fruit intake on one assessment to the following assessment. In the Stable profile, baseline fruit intake was higher than fruit intake at T1 $(\mathrm{F}(1,1.27)=4.52$; Cohen's $d=0.08 ; p<.05)$. In the Progress profile, a borderline significant increase in fruit intake was found between T2 and T3 ( F 1 , 
$50)=2.83 ;$ Cohen's $d=0.22 ; p<.10)$, and between T4 and T5 $(\mathrm{F}(1,50)=2.84$; Cohen's $d=0.22 ; p<.10$ ). A significant decrease in fruit intake was found between $\mathrm{T} 1$ and $\mathrm{T} 2(\mathrm{~F}(1,36)=4.50 ;$ Cohen's $d=0.21 ; p<.05)$, and between T4 and T5 (F (1, $36)=6.19$; Cohen's $d=0.13 ; p<.05)$ in the Regress profile. Mean fruit intake scores on each of the assessments are reported in Table 4.6.

\section{Differences in demographics}

One-way ANOVA with a Scheffé multiple-comparison test $(\alpha=.05)$ revealed a significant difference in mean age between respondents in the different transition profiles $\left(\mathrm{F}(6,376)=4.74 ; \eta^{2}=.07 ; p<.001\right)$. Respondents in the Impossible transition profile were significantly older than respondents in the Stable (Cohen's $d=0.66 ; p<.01$ ), in the Progress (Cohen's $d=0.87 ; p<.01$ ) and in the Progress then Regress (Cohen's $d=0.93 ; p<.01$ ) profiles. Chi-square analyses detected a significant difference in level of education between transition profiles $\left(\chi^{2}(12, N=\right.$ $374)=30.67$, Cramér's $V=.20 ; p<.01$ ). No significant difference was found for sex $\left(\chi^{2}(6, N=376)=5.51, n s\right)$. For level of education, pairwise comparisons were conducted. A Bonferroni procedure was used to correct for multiple testing, resulting in an alpha-level of $p<.002$. In the Progress profile, fewer respondents had a low level of education and more respondents had a medium or high level of education than respondents in the Multiple Change profile $\left(\chi^{2}(2, N=85)=\right.$ 12.00, Cramér's $V=.38 ; p<.002$ ). In the Progress then Regress profile, fewer respondents had a low or high level of education and more respondents had a medium level of education than respondents in the Impossible profile $\left(\chi^{2}(2, N)\right.$ $99)=13.55 ;$ Cramér"s $V=.37 ; p<.001)$ and in the Multiple Change profille $\left(\chi^{2}(2, N\right.$ $=80)=12.10$, Cramér's $V=.39 ; p<.002$ ). Since in our sample only few respondents were of foreign origin, differences in ethnicity were only calculated for the Stable profile compared to all other profiles. A small significant difference $\left(\chi^{2}(1, N=\right.$ $376)=3.87, r+10 ; p<.05$ ) was found, with fewer respondents of foreign origin and more respondents of Dutch origin in the Stable profile than in the other profiles together.

\section{Discussion}

The present study aimed to gain more insight into stability and changeability of stages of change by describing longitudinal stage transitions for fruit intake in an adult cohort not exposed to a planned intervention.

Several of our findings are in line with predictions from the TTM. First, the results indicate that the precontemplation and maintenance stages are the most stable stages of change, with high stability rates on all time intervals. 


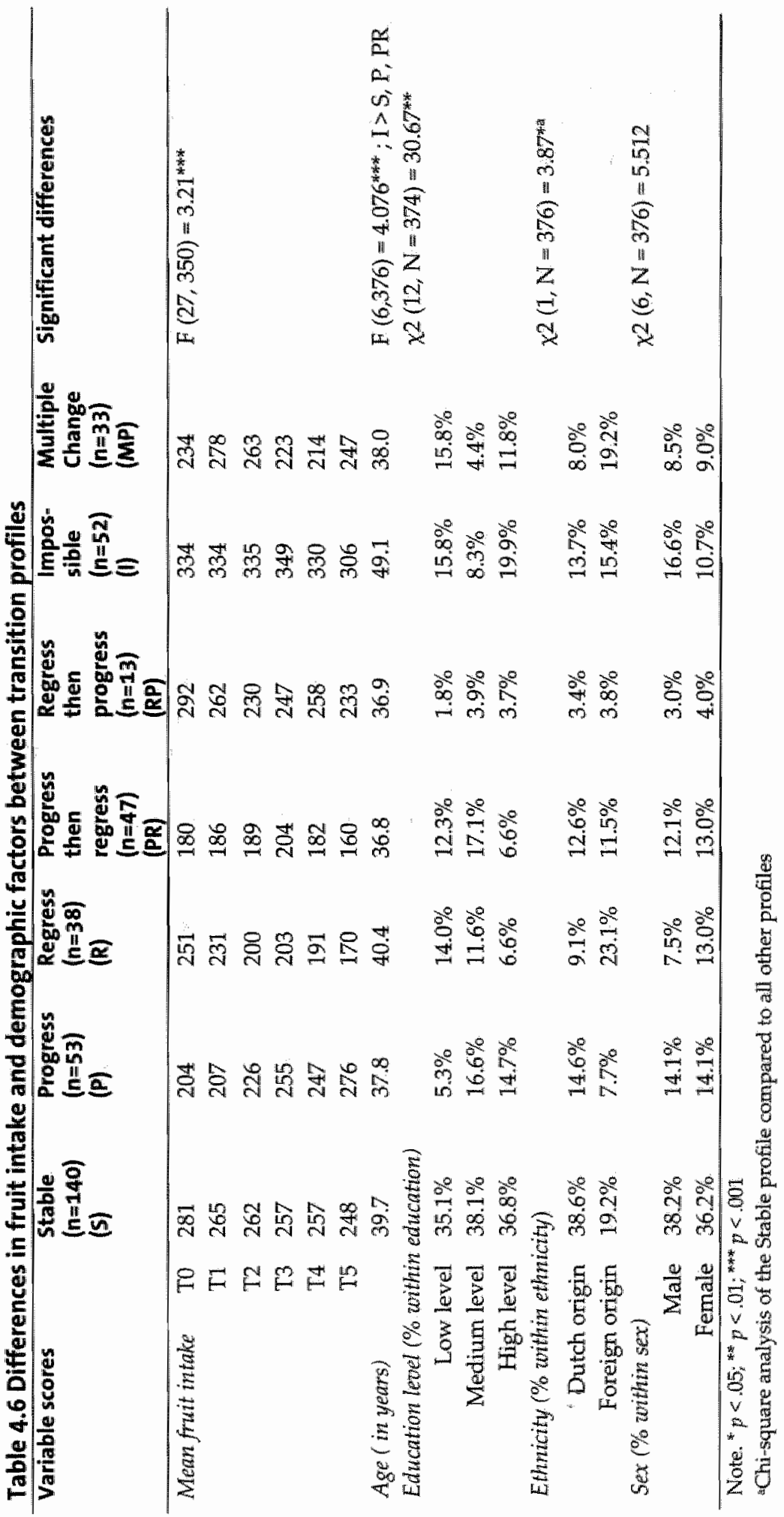


This was further confirmed by our finding that respondents in the Stable profile were mainly respondents who were in precontemplation or maintenance at baseline. The preparation stage was the least stable, which was also to be expected based on the TTM, since people in preparation intend to change their behavior in the next month.

Second, shorter time intervals generally showed higher stability. The maintenance stage exactly follows the outline as expected. Remarkably, for the preparation stage, the highest stability rate was found for the longest time interval. Since preparation is the least stable stage, it may be that respondents moved out of preparation and back during the interval between assessments.

Third, the stability of stages of change was further confirmed by means of Cohen's Kappa, showing fair to good agreement. The Kappa's found in the present study are considerably higher than the Kappa of .43 for fruit intake found by De Nooijer et al. (2005) which may be due to the longer time intervals used in that study.

A fourth encouraging finding is that in most profiles, sequences involve stage transitions to adjacent stages. This might indicate that people moving through the process of health behavior change, indeed pass through all stages as suggested by the TTM. Thus, the shorter time intervals used in the present study were helpful in detecting the most frequently occurring stage transitions.

Similar results were found in smoking cessation research in which latent transition analysis was applied, a recently developed statistical method for the analysis of longitudinal categorical data (Martin, Velicer, \& Fava, 1996; Velicer, Rossi, Diclemente, \& Prochaska, 1996). In both studies, respondents were more likely to move to adjacent stages of change than to progress or regress two stages. Velicer and coworkers (1996) also found that precontemplation and maintenance were the most stable stages of change, while Martin and colleagues (1996) found contemplation and maintenance to be the most stable stages.

Although in our study stages were rather stable, "spontaneous" stage transitions did occur, also in short time intervals. For example, although precontemplation was the most stable stage, in a time interval of two weeks, $25 \%$ of precontemplators progressed. This may offer good opportunities for interventions, since even among precontemplation, a fair proportion seem somewhat ready for change. Three explanations can be given for this instability. First, self-change may have been induced by exposure to health behavior change messages that, for example, appear in the media. Second, stage instability might result from unreliable measurement of stages of change. A third explanation might be that psychological constructs may vary over time, especially when respondents are ambivalent about such a construct, as attitudinal research has shown (Eagly \& Chaiken, 1998; Olson \& Maio, 2003).

Furthermore, the present study revealed seven patterns of change, socalled transition profiles. Compared to the dynamic typologies used by Norman 
et al. (1998), our study utilized all six measurements, which made it possible to discern the Progress then Regress profile and the Regress then Progress profile. Remarkably, when using more than one measurement within six months, it is possible to elicit stage transitions that according to the TTM are not possible to occur. One might argue that respondents in this profile may have made a mistake in reporting their stage. If so, this goes for $14 \%$ of respondents. Measuring selfreport stages of change involves two estimates, an estimate of behavior and an estimate of time. Research has been done on the accuracy of self-assessed dietary intake, and it has been argued that a dietary change staging algorithm should include more objective assessment of diet, since many people give optimistically biased self-assessments (Greene et al., 1999; Kristal, Glanz, Curry; \& Patterson, 1999; Lechner et al., 1998). Although not all respondents in the action and maintenance stages ate fruit according to recommendations, our data indicated that a majority of these respondents did. Yet, people may not only be overly optimistic about the adequacy of their diet, but also about when they made healthy dietary changes.

We found evidence for differences between respondents in different stage transition profiles in demographic characteristics and behavior changes. Interestingly, changes in fruit intake reasonably corresponded with the transition profile. For example, in the Progress profile, fruit intake increased, while in Regress fruit intake decreased. Also in other profiles, although not significant, the changes in fruit intake largely followed the pattern of stage transitions. Further exploration into differences between respondents in these transition profiles is needed.

The present study has its limitations. First, asking respondents six times about their fruit intake and stage of change might have influenced their answers. However, no overall significant changes in fruit intake over time were found and the stage distribution was rather stable over time. Second, data were collected from a random sample of an existing Internet research panel, which may have reduced external validity. In our sample, higher educated people (36\% vs. 29\%) and respondents younger than $30(36 \%$ vs. $21 \%)$ were overrepresented, while lower educated people ( $16 \%$ vs. $27 \%$ ) and respondents over 55 years old ( $15 \%$ vs. $70 \%$ ) were underrepresented, and prevalence of not eating at least two servings of fruit a day (56\% vs. $70 \%$ ) was lower compared to the Dutch adult population at large (Dutch Nutrition Centre, 1998; Statistics Netherlands, 2003). However, using the Internet panel made it possible to have repeated measurements within short time intervals with rather high response rates and might actually be one of very few methods for such tracking of stages of change. Third, although medium to high response rates were established for each of the assessments, only $51 \%$ of respondents completed all six measurements. Since age was associated with not completing all six questionnaires, this might have induced the differences between transition profiles in age. 
Beside the theoretical and conceptual questions raised in this discussion section, the results warrant practical considerations. One should be cautious using stage transition as an outcome measurement in evaluation of health promotion interventions, since "spontaneous" stage transitions in fruit intake occur frequently, even in short time intervals. Further, when tailoring interventions to stage of change, the delay between stage assessment and intervention must be minimal, otherwise interventions may be tailored to the wrong stage. However, in order to develop effective stage-tailored interventions, further research is needed into psychosocial and cognitive factors that might induce stage transitions and can discriminate between transition profiles, and to find out if similar profiles are present for other behaviors. 


\section{Chapter 5 \\ The Transtheoretical stages of change and fruit intake: real or pseudostages?}

\section{Abstract}

In the present study we explored if the pre-action Transtheoretical stages of change applied to fruit intake in fact may reflect pseudostages, i.e., a stage-like categorization of a continuous behavioral intention scale. In a longitudinal design, a cohort of 735 individuals were asked three times (baseline, 35 and 67 days later) to complete electronic questionnaires on fruit intake, stages of change and intention to increase fruit intake. Dichotomizing the continuous intention measure created an unmotivated pseudostage (intention $\leq 0$ ) and a motivated pseudostage (intention $>0$ ). These were compared to the precontemplation and contemplation stages of change. Strong associations were found between pseudostages and stages of change. Second, for a majority of respondents, stability and transitions in stages of change resembled transitions in pseudostage, while test-retest reliabilities for both measures were similar. Finally, stages and pseudostages both predicted fruit intake, but in a regression model with both measures, only pseudostages contributed significantly to the prediction of fruit intake. To conclude, the present study indicates that pre-action stages of change for fruit intake might reflect pseudostages.

Submitted for publication as: De Vet, E., De Nooijer, J., De Vries, N.K., \& Brug, J. The Transtheoretical stages of change and fruit intake: real or pseudostages? 


\section{Introduction}

The present paper explores if the Transtheoretical stages of change represent a discrete variable (as assumed in the theory) or a continuous variable that is categorized into stage-like categories, so-called pseudostages.

According to the Transtheoretical Model of behavior change (TTM; Prochaska, DiClemente, \& Norcross, 1992) behavior change is a dynamic process, which involves movement through a sequence of five discrete and qualitatively different stages, the so-called Stages of Change: precontemplation (no intention to change behavior within the next six months), contemplation (intention to change behavior within the next six months), preparation (intention to change behavior within 30 days), action (people changed from unhealthy to healthy behavior within the past six months), and maintenance (maintenance of the behavior change for more than six months) Stages of change should be characterized by a qualitative difference, which means that people in these different stages are assumed to differ on important and relevant factors (Weinstein et al., 1998). As a consequence, forward stage transition is more likely when interventions are matched to these relevant stage-specific factors. In addition, people may need a sequence of interventions to move through all stages of change suggested by the TTM.

Though the TTM has gained great popularity in recent years (as reflected in the large number of relevant publications, 444 between 2000 and 2004), the model has also been subject to criticism (Bandura, 1997; Littell \& Girvin, 2002; Smedslund, 1997; Sutton, 2000a). One important comment is that the Transtheoretical stages of change might not reflect a real staging process but merely fragment an underlying continuous variable, resulting in stage-like categories which can be created out of any continuum (Sutton, 2001; Weinstein et al., 1998). For example, stage-like categories can be created out of a continuous scale that measures planned time to action or behavioral intention.

What is the difference between a continuum and a staged approach of behavior change and why is the distinction important? In contrast to stage models, continuum models identify variables that influence action. They then combine these variables in a linear prediction equation by which the probability that this person will act is calculated, i.e., this individual is placed along an action likelihood continuum (Weinstein et al. 1998). In continuum theories two types of linear prediction equations can be found. First, a linear prediction equation without interaction terms in which the contribution of a variable is independent of the contribution of the other identified variables. According to such models it is unnecessary to match interventions since such theories do not assume the importance of targeting different social cognitive variables for different levels of motivation. For example, the Theory of Planned Behavior (Ajzen, 1991) does not 
predict that an intervention would be more effective for low intenders than for high intenders (Weinstein et al., 1998). In a second type of continuum theories interaction terms are included in the linear prediction equation, so the contribution of an independent variable to the dependent variable is conditional upon (the value of) another independent variable (Weinstein et al, 1998). For continuum theories with interaction terms, matching interventions can still be beneficial. For example, in the original Protection Motivation Theory (Rogers, 1975), perceived severity (severity of negative consequences of behavior), vulnerability (likelihood of contracting the negative consequences) and response efficacy (effectiveness of recommended health behavior to avoid negative consequences) were assumed to combine multiplicatively to arouse protection motivation (i.e., behavioral intention). This implies that when one of these components levels off to zero, protection motivation will not be increased (Boer \& Seydel, 1996). As a consequence, an intervention aimed at increasing protection motivation by increasing perceived threat should be matched to the level of perceived response efficacy (Weinstein et al., 1998). In other words, it would be of no use to increase perceived threat if an individual believes that the desired response is ineffective to reduce health risks; protection motivation would not be increased. And vice versa, if an individual perceives no threat at all, an intervention to increase response efficacy will not contribute to protection motivation either. So, matching would be beneficial although such theories provide no recommendations on what should be done first, i.e., whether a threat message should be followed by an efficacy message or that the order should be reversed. More specifically, none of the continuum theories imply the importance of intervention sequencing, while stages of change models do (Weinstein et all, 1998). So, in contrast to the TTM; no continuum theory would hypothesize that a precontemplation-matched intervention followed by a contemplation-matched intervention is more effective in changing behavior than the reverse order:

A sequence test would therefore be an ideal test to exclude the alternative explanation of pseudostages. Yet, at this point in time such a design would be complicated for several reasons. On the one hand, we need to know what a stagematched intervention must contain in order to produce progress out of that particular stage. To date, not much evidence exists for the merits of stagematching (see for reviews Adams \& White, 2003; Riemsma et al, 2003; Van Sluijs, Van Poppel, \& Van Mechelen, 2004), which may be due to the limited knowledge on stage-specific predictors that should be targeted in such an intervention (Chapter 1, De Vet, Brug, De Nooijer, Dijkstra, \& De Vries, 2005). On the other hand, difficulties would also exist with the time interval that is needed between interventions to allow progress to occur in order to test the effectiveness of intervention sequencing (Weinstein et al., 1998). It is not known for example how much time it takes after an intervention for an individual to progress to a next 
stage, or after how long an intervention maximizes effectiveness and to what extent this depends on the stage of change, behavior or other factors.

As Sutton (2000a) and Weinstein and colleagues (1998) suggest, an alternative approach to study pseudostages would be to create pseudostages out of a continuum, such as behavioral intention, and compare these pseudostages to stages of change. A common denominator, to which stages of change; pseudostages or continuous intention can be related, is motivation. Although numerous health behavior theories exist, all seem to share the notion that motivation is an important proximal determinant of health behavior (Armitage \& Conner, 2000). On a conceptual level, stage of change, in particular the pre-action stages, and behavioral intention are both concemed with motivation and may thus overlap.

To date, very few studies have specifically examined the relation between stages and intention. Kraft and colleagues (1999) categorized a continuous intention measure into three pseudostages, i.e., a low, medium and high intention pseudostages and compared these with precontemplation, contemplation and preparation stages of change on the ability to predict three variables that are conceptualized by the Transtheoretical Model, i.e.p pros, cons and self-efficacy. They found that the intention measure (either categorized as pseudostages or continuous) provided more detailed information since this measure increased the prediction of the pros, cons and self-efficacy and their results seem to be in line with a continuum model of health behavior change as well. This finding, though, might be partly related to the lack of differences between contemplators and preparators. Also, one might question what value of the contimuous intention measure would distinguish contemplators from preparators, and an alternative method would have been to create only two pseudostages, i.e., unmotivated and motivated. De Vries and Backbier (1994) did so, and created "precontemplation" and "contemplation" pseudostages by dichotomizing a continuous intention scale and found differences in attitudes and social support between these groups. They did not include a measure of stage of change, though the differences between the two pseudostages were replicated in cross-sectional studies that included a stage of change measure (De Vries, Mudde, Dijkstra, \& Willemsen, 1998).

Both studies, however, used cross-sectional designs, and no information could be derived about transitions in stages of change and pseudostages. To our knowledge, only one longitudinal study has been conducted that examined the relation between intention and stages of change for exercise (Courneya et al., 2001). They found that intention predicted forward and backward stage transitions from all stages, which could also be an indication of an underlying continuum theory of health behavior. They did not examine, however, whether the stage of change measure could be replaced by a categorized intention measure. 
Further, none of these studies specifically examined the relation of stage of change, pseudostage, or intentions with actual behavior. Given that health behavior theories share the idea that motivation, either as pseudostage or as stages of change, influences behavior, the overlap should have its reflection in the predictive validity of stages of change and pseudostages in behavior as well.

In the present study, we longitudinally explored the overlap in stages of change and a continuous intention measure fragmented into stage-like categories. We used three questions to evaluate this overlap. First, is there a strong association between stages of change and intention, and stages of change and pseudostages (categorization of intention)? Second, how stable are the stages of change over time as compared to the pseudostages, and to what extent do stability and transitions in stages of change correspond to stability and transitions in pseudostage? Third, do pseudostages and stages of change equally predict actual behavior?

The present study focused on fruit intake, since fruit intake is considered to contribute to the prevention of cardiovascular disease (Srinath Reddy \& Katan, 2004), certain cancers (Key et al., 2004), as well as obesity (Rolls et al., 2004), while fruit intake is below recommended intake levels in many countries, including the US and most countries in Europe (Serdula et al., 2004; Van der Wilk \& Jansen, 2005). In the Netherlands, approximately $70 \%$ of the population eat less than the recommended minimum amount of two servings ( $250 \mathrm{grams}$ ) of fruit per day (Dutch Nutrition Centre, 1998; Van Oers, 2002). Another reason for a focus on fruit intake, is that fruit intake represents a continuum with variance in fruit intake across stages of change, while still a clear criterion can be set to define the action stage, based on the recommended intake level.

\section{Method}

\section{Participants and procedures}

Potential participants were identified from a random sample of 1,500 people aged 18 years and older from a Dutch Internet research panel. At the time of the study, the size of the entire panel was about 8,165 persons. People were invited by an email letter to participate in a longitudinal cohort study. Respondents could indicate their willingness to participate in the entire study by completing the first electronic questionnaire (TO). Completed questionnaires were returned by 929 respondents. Thirteen respondents were excluded from the study because stage of change $(n=1)$ or fruit consumption $(n=12)$ could not be calculated. Of the 
remaining 916 respondents (response rate $61 \%$ ), for each stage of change at baseline, $20 \%$ was selected randomly to participate in a different study. The remaining 735 respondents were sent a second electronic questionnaire 35 days after baseline ( $\mathrm{T} 1, \mathrm{~N}=610$, response rate $83 \%$ ). The third assessment took place 32 days after $\mathrm{T} 1(\mathrm{~T} 2, \mathrm{~N}=592$, response rate $81 \%)$. In total, $73 \%(\mathrm{~N}=538)$ of the respondents completed all three questionnaires. Respondents who did not complete all three questionnaires were significantly younger $(\mathrm{F}(1,735)=24.20 ; p$ $<.001)$, were higher educated $\left(\chi^{2}(2, N=732)=6.50, p=.04\right)$, and were more often male $\left(X^{2}(1, N=735)=6.50, p=.04\right)$ than respondents who did complete all questionnaires. No differences between completers and non-completers were found with respect to ethnicity, pre-action stages of change, intention, pseudostage or fruit intake.

Mean age was $37.5(\mathrm{SD}=14.1)$ years, and women and men were equally represented ( $51 \%$ vs. $49 \%) ; 17 \%, 48 \%$ and $36 \%$ reported a low, medium and high level of education, respectively. Most respondents were of Dutch origin $(90.1 \%)$. Mean fruit intake was 260 ( $\mathrm{SD}=163$ ) grams per day, and $57 \%$ of the respondents ate less fruit than the recommended minimum intake level of 250 grams a day.

Respondents used a personal login code to open and complete the questionnaires. All questionnaires had to be completed within one week after the emailed completion request. Respondents' answers were automatically entered into a data file, and after the deadline had passed by, the respondents could not enter the questionnaire anymore. Answers could not be saved, and the questionnaire could not be completed more than once.

\section{Measures}

Fruit intake was assessed with a 14-item food frequency questionnaire (FFQ), validated as compared to a 7-day dietary record $(r=.51 ; p<.001$; Van Assema et al., 2002) and biomarkers for fruit consumption levels, i.e., blood levels of total carotenoids $(r=.34 ; p<.001)$, vitamin $C(r=.27 ; p<.001)$ and betacryptoxanthin $(r=.42 ; p<.001$, Bogers et al., 2004). Respondents were asked about consumption during the previous month. The FFQ included questions about frequency of consumption (from "never/less than once per month" to "7 days a week $^{\prime \prime}$ ) and number of servings (pieces or small bowls [for small fruit such as berries] per day that a certain fruit (juice) was consumed). Separate questions were included for the most common fruits in the Netherlands (citrus fruit, such as oranges and grapefruit; apples or pears; bananas), for an important seasonal fruit (tangerines), for freshly squeezed or other unsweetened fruit juice, and for "other fruit including preserved fruit". To calculate total fruit consumption, serving sizes were first transformed to grams based on standard mean weights of the fruits. 
Subsequently, for each fruit category frequency of consumption was multiplied with serving size and the results were added up and divided by seven to obtain a fruit intake score in grams per day.

Stage of change was assessed consistent with Nigg and colleagues (1999) and Laforge et al. (1999) by asking respondents to select the most suitable statement out of five statements each representing a stage of change: "I do not eat at least two servings of fruit every day, and I do not intend to change this within the next six months" (precontemplation), "I do not eat at least two servings of fruit every day, but I intend to change this within the next six months" (contemplation), "I do not eat at least two servings of fruit every day, but $I$ intend to change this within the next month" (preparation), "I eat at least two servings of fruit every day, and I've started doing this in the last six months" (action), and "I eat at least two servings of fruit every day, and I have done so for more than six months ${ }^{\prime \prime}$ (maintenance).

Intention was assessed with two items: "Do you intend to achieve eating at least two servings of fruit each day?" (certainly not $(-2)$ to certainly yes $(+2)$ ) and "How sure are you that you want to start eating at least two servings of fruit each day?" (certainly not (-2) to certainly yes (+2)). Cronbach's $\alpha$ for the two items was .93 , and test-retest reliability with a two-week interval showed a Pearson correlation of $.83(\mathrm{p}<.001)$. A mean score for intention was computed.

Demographic variables included sex, age, education and ethnicity, and were only assessed at baseline.

\section{Data analyses}

The analyses were only conducted for respondents in the pre-action stages of change, since these respondents can validly express an intention to eat at least two servings of fruit each day. First, the contemplation and preparation stages were combined as outlined in the introduction and because earlier research on stages of change and diet found indications that a distinction between the contemplation and preparation stages might be unnecessary. Frequently these stages are merged for nutrition behaviors (cf., Brug \& Van Assema, 2000; Campbell et al., 1999).

T-tests were conducted to test for differences in mean intention scores between precontemplation, and contemplation/preparation. Next, the continuous intention measure was divided into two stage-like categories creating an unmotivated and motivated pseudostage. Respondents with a mean intention score lower or equal to zero were classified as unmotivated. Respondents with a positive mean intention score were classified as motivatéd. 
First, the proportion of respondents classified in the same pseudostage and stage of change (precontemplation and unmotivated, or contemplation/ preparation and motivated) was calculated. Next, the strength of the association between stage of change and pseudostage was computed for each assessment using the Phi-coefficient ( $r \phi$ ) for $2 \times 2$ tables (Siegel \& Castellan, 2002). According to Cohen's guidelines for interpretation of correlations, a large effect size was defined as a correlation larger than or equal to 50 . A correlation between .30 and 50 is regarded as a medium effect size, and a correlation between .10 and .30 is defined as a small effect size (Cohen, 1988).

Between two assessments, respondents in the unmotivated pseudostage and precontemplation stage could progress (moved forward) or remain stable (no stage change), Respondents in the motivated pseudostage and contemplation/preparation stage of change respondents could regress (moved backward) or remain stable between two assessments. As expressed before, only respondents in pre-action stages of change can validly express an intention and therefore respondents originally in pre-action who progressed to action on the next assessment were excluded from the longitudinal analyses. In both time intervals, $8 \%$ of the respondents in precontemplation progressed to action/maintenance. For contemplation/preparation, this percentage totals $22 \%$ and 19\% for T0-T1 and T1-T2, respectively. First, the Phi coefficient was used to evaluate the stability (test-retest reliability) in the stages of change and the pseudostages separately for both time intervals. Next, Cohen's Kappa was used to evaluate whether transitions and stability in stages of change corresponded to the transitions in pseudostages. Generally, a Kappa $>.75$ is considered excellent agreement, a Kappa between .40 and .75 as fair to good and a Kappa $<.40$ as poor (Fleiss, 1981).

Finally, two series of linear regression analyses were done to test whether stages of change and intention, and stages of change and pseudostages predicted fruit intake. First, stage of change at TO and intention at TO were entered separately in the analyses to predict fruit intake at T1. Next, both variables were entered simultaneously. Second, stage of change at T0 and pseudostage at TO were entered separately in the analyses to predict fruit intake at T1. Subsequently, both were entered simultaneously. Similar analyses were repeated for the T1-T2 time interval. The variance inflation factor (VIF) was used to evaluate multicollinearity in the multiple regression analyses. If the variables in the model reveal an average VIF larger than 1, multicollinearity is indicated (Bowerman and O'Connell, 1990). 


\section{Results}

\section{Intention, stages of change and pseudostages}

At all three time points, $t$-tests showed significant differences in intention between the stages of change, with a lower intention in precontemplation than in contemplation/preparation (see Table 5.1).

Table 5.1 Mean (SD) intention scores for stages of change for three assessments

\begin{tabular}{lccc} 
& PC & C/PR & t-value \\
\hline Intention T0 & $-0.44(0.93)$ & $0.92(0.76)$ & $-17.89^{* * * *}$ \\
Intention T1 & $-0.60(1.01)$ & $0.89(0.77)$ & $-16.10^{* * *}$ \\
Intention T2 & $-0.75(0.99)$ & $0.75(0.80)$ & $-15.49^{* * *}$ \\
\hline
\end{tabular}

Note. Significant at *** $p<.001 ; \mathrm{PC}=$ precontemplation, $\mathrm{C} / \mathrm{PR}=$ contemplation/preparation

The distribution of respondents on the stages of change and pseudostages are presented in Table 5.2 . Of the precontemplators, $81 \%, 86 \%$ and $90 \%$ were correctly classified into the unmotivated pseudostage at T0, T1 and T2 respectively. Of the contemplators/preparators, $82 \%, 78 \%$, and $72 \%$ were correctly classified into the motivated pseudostage at $\mathrm{T} 0, \mathrm{~T} 1$, and $\mathrm{T} 2$. For all assessments a strong effect size was found for the association between stages of change and pseudostages $\left(r_{\phi}=.63\right.$ at $\mathrm{T} 0$ and $\mathrm{T} 2$, and $r_{s}=.64$ at $\mathrm{T} 1$, all $\left.p<.001\right)$.

Table 5.2 Correspondence between stages of change and pseudostages at baseline

\begin{tabular}{rlr}
\hline & \multicolumn{1}{c}{ Pseudostage } \\
\cline { 2 - 3 } Stage of change (\% within Stage) & Unmotivated & Motivated \\
Precontemplation & $196(81.0 \%)$ & $46(19.0 \%)$ \\
Contemplation/preparation & $47(18.2 \%)$ & $211(81.8 \%)$ \\
\hline
\end{tabular}

Note. Only results of T0-T1 are presented in a table in case similar results were found for both time intervals. Tables about the T1-T2 interval can be obtained from the authors

\section{Stability and transitions in stages of change and pseudostages}

Of the respondents in the precontemplation stage, $70 \%$ and $80 \%$ remained stable, compared to $85 \%$ and $92 \%$ of the respondents in the unmotivated pseudostage between $\mathrm{T} 0-\mathrm{T} 1$ and $\mathrm{T} 1-\mathrm{T} 2$, respectively. Further, $22 \%$ and $12 \%$ of the 
precontemplators progressed to contemplation/preparation, compared to $15 \%$ and $8 \%$ of the unmotivated respondents who progressed to the motivated pseudostage in the consecutive time intervals.

Of the respondents in the contemplation/preparation stage, $66 \%$ and $71 \%$ remained stable, compared to $79 \%$ and $80 \%$ of the respondents in the motivated pseudostage between $\mathrm{T} 0-\mathrm{T} 1$ and $\mathrm{T} 1-\mathrm{T} 2$, respectively. Further, $12 \%$ and $10 \%$ of the respondents in contemplation/preparation regressed to precontemplation, compared to $21 \%$ and $20 \%$ of the motivated respondents who regressed to the unmotivated pseudostage between the consecutive time intervals.

Test-retest reliabilities for the two intervals varied from $\gamma_{\phi}=.61$ to .67 for stage of change and from $r \omega=.64$ to .73 for pseudostage (all $p<.001$ ). Overall, $73 \%$ and $75 \%$ of respondents had similar transitions for pseudostage as for stage of change between $\mathrm{T} 0$ and $\mathrm{T} 1$, and between $\mathrm{T} 1$ and $\mathrm{T} 2$ respectively (both time intervals $\mathrm{K}=.59 ; p<.001$, see Table 5.3).

Table 5.3 Transitions in pseudostages and stages of change between TO-T1

\begin{tabular}{|c|c|c|c|c|}
\hline \multirow[b]{2}{*}{$\begin{array}{l}\text { Stage of change } \\
\text { transition (\% within } \\
\text { stage of change) }\end{array}$} & \multicolumn{4}{|c|}{ Pseudostage transition } \\
\hline & $\begin{array}{l}\text { Unmotivated } \\
\text { stable }\end{array}$ & $\begin{array}{l}\text { Unmotivated } \\
\text { progress }\end{array}$ & $\begin{array}{l}\text { Motivated } \\
\text { stable }\end{array}$ & $\begin{array}{l}\text { Motivated } \\
\text { regress }\end{array}$ \\
\hline PC stable a & $123(90.4 \%)^{6}$ & $3(2.2 \%)$ & $3(2.2 \%)$ & $7(5.1 \%)$ \\
\hline PC progress & $9(20.9 \%)$ & $16(37.2 \%)$ & $13(30.2 \%)$ & $5(11.6 \%)$ \\
\hline C/PR stable & $15(10.3 \%)$ & $9(6.2 \%)$ & $108(74.5 \%)$ & $13(9.0 \%)$ \\
\hline C/PR regress & $11(42.3 \%)$ & - & $5(19.2 \%)$ & $10(38.5 \%)$ \\
\hline
\end{tabular}

Note. ${ }^{\mathrm{PC}}=$ precontemplation, $\mathrm{C} / \mathrm{PR}=$ contemplation/preparation

to corresponding transitions are printed in italics

\section{Predicting fruit intake by stage of change and pseudostages}

Between T0 and T1, stages of change and intention separately predicted fruit intake, but intention explained a larger proportion of the variance in fruit intake than stages of change (Table 5.4). When stage of change and intention were entered in one analysis, only the association of intention with fruit intake remained significant. The effect of stage of change was reduced to insignificance with a negative beta and an average VIF of 1.52 . The same pattern was found in the analyses with pseudostages instead of a continuous intention measure, with an average VIF of 1.49 (Table 5.5).

Between T1 and T2, intention but not stages of change predicted fruit intake. In the multiple linear regression analysis with the continuous intention measure and stages of change, the association of intention with fruit intake 
remained significant. Also stages of change predicted fruit intake, but with a negative beta. The average VIF was 1.77 . In the simple as well as the multiple regression analyses with pseudostages instead of a continuous measure, pseudostages, but not stages of change predicted fruit intake. The average VIF of the multiple linear regression analyses was 1.63 .

Table 5.4 Linear regression analyses for stage of change and intention on post-test fruit intake

\begin{tabular}{lrllll}
\hline TO-T1 & & B & SE (B) & B & Model $\mathbf{R}^{2}$ \\
\cline { 3 - 6 } Simple & Stages of Change & 28.51 & 10.39 & $.11^{* *}$ & .36 \\
Intention & 24.26 & 4.83 & $.20^{* * * *}$ & .39 \\
Multiple & Stages of Change & -5.78 & 13.07 & -.02 & .39 \\
& Intention & 25.97 & 6.20 & $.22^{* * *}$ & \\
T1-T2 & & & & & \\
Simple & Stages of Change & 10.68 & 10.79 & .04 & .48 \\
& Intention & 20.49 & 4.72 & $.19^{* * * *}$ & .51 \\
Multiple & Stages of Change & -28.13 & 13.42 & $-.11^{*}$ & .52 \\
& Intention & 28.44 & 6.04 & $.26^{* * *}$ & \\
\hline
\end{tabular}

Note. Significant at " $p<.05 ; " p<.01 ; " * p<.001$

The analyses of the TO-T1 and the T1-T2 interval are corrected for fruit intake at T0 and T1, respectively

Table 5.5 Linear regression analyses for stage of change and pseudostage on posttest fruit intake

\begin{tabular}{lrllll} 
To-T1 & & & & & \\
Simple & & SE (B) & B & Model R $^{2}$ \\
\cline { 3 - 6 } & Stages of Change & 28.51 & 10.39 & $.11^{* * *}$ & .36 \\
Multiple & Pseudostage & 48.63 & 10.45 & $.19^{* * * *}$ & .38 \\
& Stages of Change & -1.75 & 13.08 & -.007 & .38 \\
T1-T2 & Pseudostage & 49.75 & 13.37 & $.19^{* * * *}$ & \\
Simple & & & & & \\
& Stages of Change & 10.68 & 10.79 & .04 & .48 \\
Multiple & Pseudostage & 35.59 & 11.06 & $.14^{* * * *}$ & .50 \\
& Stages of Change & -16.99 & 13.75 & -.07 & .50 \\
& Pseudostage & 46.81 & $\mathbf{1 4 . 3 1}$ & $.18^{* * *}$ & \\
\hline
\end{tabular}

Note. Significant at " $p<.05 ; " p<.01 ; " * p<.001$

The analyses of the T0-T1 and the T1-T2 interval are corrected for fruit intake at T0 and T1, respectively 


\section{Discussion}

In the present study we found several indications that pre-action stages of change represent a categorization of a continuous intention measure instead of discrete stages. First, strong correlations were found between stages of change and pseudostages. Second, for a majority of respondents, stability and transitions in stages of change resembled transitions in pseudostage, while test-retest reliabilities for both measures were approximately similar. Finally, when both stages of change and pseuclostages are entered in one analysis to predict fruit intake, multicollinearity was found. This may indicate that stages and pseudostages might reflect the same concept.

One might argue that fruit intake was better predicted by pseudostages than by the stages of change, since the TTM assumes that the prediction of behavior involves a separate stage, i.e., the action stage. Previous studies, though, have showed a linear trend through the stages of change for dietary behaviors, e.g. fat, fruit and vegetable intake (e.g., Burke et al., 2000; Ma et al., 2003). Such linear changes might also be regarded as evidence for an underlying continuum model of behavior change, since a real stage model would show discontinuity patterns in relevant variables across the stages (Sutton, 2000b).

When further reviewing the results that stages of change research has revealed to date, most could also be in line with pseudostage models. First, in crosssectional designs not only actual behavior but several psychosocial variables tend to show a linear increase instead of a discontinuity pattern across the stages of change (Armitage \& Arden, 2002; Sutton, 2000b). For example, self-efficacy tends to increase monotonically through the stages of change with low self-efficacy scores in precontemplation and high self-efficacy scores in action and maintenance for various health-related behaviors, e.g.r smoking, fruit and vegetable intake, exercise (Herrick, Stone, \& Mettler, 1997; Horacek et al., 2002; Kraft, Sutton, \& McCreathReynolds, 1999). Second, in longitudinal research the evidence for stage-specific transition determinants has been limited. For example, self-efficacy has been found to predict all early stage transitions in exercise and fruit intake (Courneya et al., 2001; De Vet, De Nooijer et al., 2005a). Third, some studies showed that stages of change could be subdivided into several other stages (Dijkstra et al., 1997; Kremers et al., 2001; Norman et al., 2000; Velicer et al., 1995). For example, Norman and colleagues (2000) distinguished four subtypes in the precontemplation stage, in the contemplation stage as well as in the preparation stage, resulting in 12 pre-action stages. As a result of such an ongoing fragmentation, stages may in an increasing degree resemble a continuum rather than clear-cut discrete homogeneous stages of change. Fourth, match-mismatch studies have shown that people also benefit from an intervention that is mismatched to their current stage of change (Dijkstra, De Vries, Roijackers, \& Van Breukelen, 1998; Quinlan \& McCaul, 2000). For example, Quinlan and McCaul (2000) found that smoking precontemplators receiving an action-matched intervention showed a higher intention to quit, attempted more 
often to decrease daily smoking, attempted more often to quit, and refrained from smoking longer than precontemplators in a precontemplation-matched intervention condition.

Our results seem to further support that stages of change are pseudostages. However, limitations must be kept in mind. For example, one could debate where to put the cut-off point in the continuous intention measure in order to distinguish the motivated and unmotivated pseudostages. A different cut-off point may lead to different results. We believe, however, that the negative-positive split in intention is the most plausible way to discriminate unmotivated from motivated respondents and is also consistent with the study by De Vries and colleagues (1994). Another limitation might be the restriction to the early, motivational, stages of behavior change in the present study. It has been argued elsewhere that the Transtheoretical (pre-action) stages may not be valid, but that does not imply that behavior change is a continuous process. For example, Bagozzi's goal theory (Bagozzi \& Edwards, 1998) and the Health Action Process Approach (Schwarzer, 1992b) suggest that behavior change follows three phases: a motivational, a volitional and an action phase. According to such models, one should start increasing people's motivation to change (comparable to the Transtheoretical pre-action stages taken together), then focus on translating motivation into actual behavior change, for example by implementation intentions or action planning; finally, it is important to prevent relapse. Further research is necessary to test the true stage-like character of such three phase-models.

It should be noted that the present study used two items to construe intention. Courneya and colleagues (2001) argued that there are two kinds of intention, i.e., choice intention (what a person intends to do) and behavioral intention (how strongly the person intends do it). In their view, the choice intention might be comparable to stages of change, but the behavioral intention differs from the stages since behavioral intention may be seen as a kind of commitment to the choice intention (Courneya et al., 2001). However, Courneya et al. (2001) showed that also behavioral intention was predictive of all forward and backward stage transitions and might as well be the same concept. In this study, we therefore combined both (highly internally consistent) indicators of intention. Moreover, repeating the analyses with only the choice intention item, revealed no differences in the results.

So, either way, intention and stage of change might overiap. We are aware that our single longitudinal study cannot provide a definitive view on the issue, and more research is needed to more definitely test the pseudostage-like character of TTM stages of change. Nevertheless, as is called for in recent years, our study did attempt to look for commonalities among theories and concepts in health behavior change instead of highlighting the differences (Kruglanski, 2001; Marsh, 1994; Nigg, Allegrante, \& Ory, 2002). Our results provide indications that a continuous intention measure might be the better candidate in predicting fruit intake, and using a continuous variable instead of the categorical pre-action stages would probably pick-up more subtle changes in fruit intake. 



\section{Chapter 6}

\section{Testing the Transtheoretical model for fruit intake: comparing web-based individualized stage-matched and stage-mismatched feedback}

\section{Abstract}

To test the Transtheoretical model applied to fruit intake, precontemplators and contemplators (as determined in a pretest) were randomly assigned to receive web-based individualized precontemplation-matched, contemplation-matched or action-matched fruit promotion feedback. Immediately and one week after reading the letter, post-test measures were obtained. Precontemplators receiving the action-matched feedback had marginally significantly higher fruit intake compared to those in the precontemplation-matched condition, but no differences were found in intention and stage progression. Contemplators in the precontemplation-matched condition had higher intentions than contemplators in the contemplation-matched condition, but no differences between the conditions were found for fruit intake and stage progression. It can be concluded that the present study failed to show superiority of stage-matched information in the promotion of fruit intake.

Submitted for publication as: De Vet, E., De Nooijer, J., De Vries, N.K., \& Brug, J. Testing the Transtheoretical model for fruit intake: comparing web-based individualized stage-matched and stage-mismatched feedback. 


\section{Introduction}

The Transtheoretical model of behavior change (TTM) provides an intuitively appealing framework for understanding the difficulties and complexities of the process of health behavior change. The attractiveness seems to result from the TTM's core organizing construct, the stages of change, which represents a series of five discrete qualitatively distinct stages of change: precontemplation (no intention to change behavior within the next six months), contemplation (intention to change behavior within the next six months), preparation (intention to change behavior within 30 days), action (people changed from unhealthy to healthy behavior within the past six months), and maintenance (maintenance of the behavior change for more than six months; Prochaska \& DiClemente, 1983; Prochaska \& Velicer, 1997a). Although the TTM has gained great popularity in recent years, questions have been raised about the validity of the staging character (Bandura, 1997; Sutton, 2001; Weinstein et al., 1998).

To reflect a true staging character, stages should be qualitatively different (Weinstein et al., 1998). This means that determinants of progress from precontemplation to contemplation should be different from determinants of transition from contemplation to preparation; determinants of change should be stage-specific. TTM components, such as decisional balance (individuals' weighing of the importance of pros and cons of a behavior), self-efficacy (individuals' situation-specific confidence in coping with high-risk situations for unhealthy habits), and the processes of change (experiential and behavioral activities that individuals use to progress to later stages of change) can be regarded as potential stage transition determinants (Prochaska, 1994; Prochaska et all., 1988; Velicer et al., 1985; Velicer et al., 1990).

If stages are qualitatively different, individuals in different stages should benefit most from so-called stage-matched interventions; interventions matched to the relevant stage-specific factors (Weinstein et al., 1998). Eventually, behavior change is the desired outcome, but for a brief intervention it is assumed that stage progress is a relevant goal (Prochaska \& Velicer, 1997a). Stage-matched interventions should ideally combine two strategies, (1) targeting, delivering a common content to all members of a particular stage group, and (2) tailoring, providing person-specific feedback to individuals within a stage group (Rakowski et al, 1998). This combination of stage-matching and individualization is considered necessary since research has shown large within-stage differences in pros, cons and temptations (Velicer et al., 1995). Thus, relying only on stagetargeting may not be sufficient to obtain the desired health outcomes (Velicer et al., 1999).

If the stages of change construct is valid, interventions matched to an individual's current stage of change should be more effective than interventions 
mismatched to stage of change (i.e, matched to a different stage; Sutton, 2001; Weinstein et al., 1998). Comparing matched and mismatched interventions would therefore provide a strong test of the TTM. To our knowledge, only three match mismatch studies have been reported to date, which revealed conflicting results (Blissmer \& McAuley, 2002; Dijkstra et al., 1998; Quinlan \& McCaul, 2000).

In a match-mismatch test for physical activity promotion, Blissmer and McAuley (2002) randomized participants into one of four conditions, i.e., a stagematched (a personalized letter and an appropriate stage-specific generic manual), a stage-mismatched (a personalized letter and a similar manual but inappropriate to the individual's current stage), a standard care and a control condition. The stage-matched condition outperformed the stage-mismatched condition on physical activity. However, the study sample was relatively active at baseline with large numbers of respondents in preparation, action and maintenance: Furthermore, the analyses did not discriminate between stage of change and type of mismatch. So, an actor in an action-matched condition was compared to a precontemplator in a maintenance-matched condition, but the interaction of stage and condition was not analyzed.

Quinlan and McCaul (2000) did test for an interaction between stage and condition. In a randomized trial, precontemplators were assigned to a matched condition (in which smokers were stimulated to think about quitting) or to a mismatched condition, which contained action-oriented information typically suitable for smokers who are ready to quit. No statistically significant group differences were found, though the action-oriented (mismatched) information performed better than the matched information in stage progression, intention to quit, and percentage of smokers who quit or decreased daily smoking. Quinlan and McCaul (2000), however, did only include a targeting strategy and not a tailoring strategy, which might have affected their results.

Dijkstra and colleagues (1998) included such a tailoring strategy in a match-mismatch test on smoking cessation. In a $4 \times 4$ design, so-called immotives (smokers with no intention to quit within five years), precontemplators, contemplators and preparators were randomized into an outcome information condition (immotive-matched), self-efficacy enhancing information (preparationmatched), combined outcome and self-efficacy information condition (precontemplation as well as contemplation-matched) and a control condition. Only weak evidence was found for the merits of stage-matching, i.e., borderline significant higher 7-day cessation rates and a higher proportion of stage progressors were found in smokers with a high readiness to quit (contemplators and preparators combined) in the matched condition compared to the mismatched condition. Remarkably, precontemplators and contemplators were assumed to benefit from the same information, so contrary to the TTM, no stagespecific predictions for these stages were made ( $\mathrm{cf}_{\text {., }}$ Sutton, 2000a). Further, only outcome and self-efficacy information was given, while the TTM proposes that 
also processes of change might be relevant for guiding stage transitions (Prochaska \& Velícer, 1997a; Prochaska et al., 1988).

In summary, match-mismatch tests of the Transtheoretical model have revealed conflicting results to date. However, some useful lessons can be learned from these studies. First, inclusion of a talloring strategy in addition to a stagetargeting may indeed be useful (Dijkstra et al,, 1998). Second, matching to or tailoring on decisional ballance and self-efficacy may not be sufficient, and therefore, the TTM processes of change may also need to be used for stagematched interventions. Third, the (lack of) differences in effects between matched and mismatched interventions may depend on the extent and direction of the mismatch. For example, providing precontemplators with action-matched feedback may be a larger mismatch than the provision of contemplation-matched feedback. Also, mismatched feedback matched to an earlier stage of change (a backward mismatch) may result in different effects than feedback matched to a later stage (a forward mismatch, of, Quinlan \& McCaul, 2000). Fourth, all three studies had a delay between stage assessment and intervention delivery. Earlier studies showed that stage change occurs frequently in natural settings, also over short time intervals (De Nooijer, Van Assema et al., 2005; De Vet, De Nooijer et al., 2005b). As a result, an intervention matched to a stage of change measurement of some days or even weeks earlier, may no longer be matched at the time of intervention due to stage instability. Providing computer-generated feedback immediately after stage assessment can prevent such unplanned mismatching.

These insights were combined in the present study. In a randomized trial we examined whether stage-matched individualized feedback induces more stage progress, more positive intentions to eat enough fruit, and higher fruit intakes than stage-mismatched feedback. More specifically, we tested:

1. In precontemplators, whether web-based tailored individualized precontemplation-matched feedback was more effective than contemplation-matched or action-matched feedback.

2. In contemplators, whether web-based tailored individualized contemplation-matched feedback was more effective than precontemplation-matched or action-matched feedback.

The match-mismatch test is related to fruit intake, since ample fruit intake is considered to be an important everyday health behavior that may contribute to the prevention of obesity (Rolls et al., 2004), cardiovascular diseases (Srinath Reddy \& Katan, 2004), and certain cancers (Key et al., 2004), whereas in most countries, including the US and most countries in Europe, a majority of people do not meet the guidelines for sufficient fruit intake (Serdula et al., 2004; Van der Wilk \& Jansen, 2005). Since fruit intake is recommended on a daily basis, it may therefore be suitable to study stage transitions and changes in consumption. 


\section{Method}

\section{Participants and procedures}

Potential respondents were recruited from a random sample of 5,026 people from a Dutch Internet research panel. At the time of study the entire Internet research panel comprised 48,718 individuals. Respondents could indicate their willingness to participate in the study, by completing a screening instrument assessing stage of change ( $\mathrm{N}=3,257$, response rate $65 \%$ ). Respondents in the preparation, action and maintenance stages of change and respondents aged 18 years or younger were excluded for further participation ( $N=2,297,71 \%$ ). The screening revealed 593 and 367 eligible respondents in the precontemplation and contemplation stages respectively. Based on a power-analysis, 408 respondents in the precontemplation stage were randomly selected to participate. All contemplators participated since the screening revealed a sufficient number of contemplators. Respondents in the precontemplation and contemplation stages, separately, were randomly assigned to one of three feedback conditions. In total, 775 individuals were included to participate further, of which 257, 256 and 262 individuals were randomly assigned to the precontemplation-matched, contemplation-matched and action-matched condition, respectively.

Two days after screening (T0), the 775 participants were sent the first questionnaire ( $\mathrm{T} 1$ ), received web-based tailored feedback, and were assessed a second time (T2). The first phase of the study (T1 and T2) had to be completed in one go within one week from receiving the questionnaire $(N=636$; response rate $82 \%)$. Respondents received the T3 questionnaire exactly one week after they had completed $\mathrm{T} 1$ and $\mathrm{T} 2(\mathrm{~N}=573$; response rate $90 \%)$.

\section{Study conditions}

Generating tailored feedback involves several steps. First, respondents complete a diagnostic tool and the answers are transferred to a data file. Second, the tailoring program links the diagnostic results to a feedback message library file that contains feedback messages for all possible diagnostic results, and selects the appropriate messages for each individual respondent and generates the individualized feedback (Brug, Campbell, \& Van Assema, 1999; Brug, Oenema, \& Campbell, 2003). Each respondent therefore receives feedback that is dependent on the responses to the questions in the diagnostic tool. In the present study, this feedback was presented via Internet in the form of a computer-generated individually tailored feedback letter, which contained stage-specific feedback and information. In each feedback condition, the content as well as the 
communication about the content were stage-specific following TTM recommendations. The letter addressed the respondents by name and started with a statement that the information was tailored to their personal situation.

\section{Precontemplation-matched feedback (PCM)}

The PCM feedback focused on the pros (e.g., health profits, weight regulation etc; cf., Prochaska, 1994) as well as the precontemplation stage-specific processes of change, i.e, the consciousness-raising (websites with recipes), dramatic relief (increase emotional reactions followed by strategies to reduce concern), and environmental re-evaluation (how one can serve as a role model for children and partners etc.) processes of change (Prochaska, 2000; Prochaska \& Velicer, 1997a). Directive feedback was avoided by encouraging cognitive or mental activities instead of behavioral actions (Jordan \& Nigg, 2002; Prochaska, DiClemente, \& Norcross, 1992). At the end of the feedback letter, respondents were asked to think about the feedback they had read and to think if they would be willing to eventually consider change in the future.

\section{Contemplation-matched feedback (CM)}

The CM feedback contained communication that combined cognitive with behavioral activities and discussed the cons of fruit intake (e.g., cost, taste, purchasing, preparing and storing fruit; $c f_{.,}$Prochaska, 1994), the contemplationspecific self-reevaluation process of change (imagining what would change if one would start to eat at least two servings of fruit each day; cf., Prochaska \& Velicer, 1997a) and coping with situations that may make eating sufficient amounts of fruit difficult (e.g, during weekends, in winter, when experiencing (emotional) distress). Contemplators may rely more on the perception of difficulties than on actual experience with difficult situations, so the feedback was aimed at building self-efficacy through the use of cognitive skills (cf, Jordan \& Nigg, 2002; Plummer et al., 2001; Prochaska \& Velicer, 1997a). At the end of the feedback letter, respondents were asked to think about the feedback and to consider if they would be willing to change in the near future.

\section{Action-matched feedback (AM)}

The AM feedback communicated behavioral skills and activities directed at maintaining the behavioral changes respondents had made and how to incorporate these changes in their daily lives in order to facilitate habit formation. The focus in this feedback was on increasing self-efficacy by means of behavioral skills (Prochaska \& Velicer, 1997a), and increasing the application of actionspecific processes of change such as counterconditioning (substituting unhealthy habits by fruits, such as snacking), gathering social support, reinforcement management by developing simple rewards for eating fruits (e.g, felt positive consequences of eating fruits according to recommendations) and stimulus 
control by adding cues to eat fruits (e.g., placing a filled fruit bowl on the table cf., Jordan \& Nigg, 2002; Prochaska \& Velicer, 1997a). At the end of the feedback letter, respondents were asked to think about the feedback and to try to incorporate some of these tips in their daily lives.

\section{Measures}

Fruit intake was measured with a validated 14-item food frequency questionnaire (FFQ) assessing the frequency (how many days a week) and amount (pieces or small bowls [for small fruit such as berries] per day) of consumption with separate questions for the most common fruits in the Netherlands, i.e., citrus fruit, apples and pears, bananas, freshly squeezed or unsweetened fruit juice, tangerines, and for other fruit including preserved fruit. From this information daily fruit intake in grams was calculated. The reliability and validity of the FFQ has been described in detail elsewhere (Bogers et al., 2004; Van Assema et al, 2002)

Stage of change was operationalized by a single-item algorithm consistent with Armitage and Arden (2002) and Laforge et al. (1999) and adlapted to fruit intake. First, a description of the Dutch recommendations for fruit intake was presented. Respondents were asked whether they ate at least the recommended two servings of fruit each day by selecting one of five statements each representing a stage of change: "No, and I do not intend to change this within the next six months" [precontemplation], "No, but I intend to change this within the next six months" [contemplation], "No, but I intend to change this within the next month" [preparation], "Yes, and I have started doing so in the last six months" [action], "Yes, and I have done so for more than six months" [maintenance]. Stage of change was assessed at all measurements.

Intention was assessed with three items at $\mathrm{T} 2$, i.e., Do you intend to eat at least two servings of fruit each day ( 2 [definitely not] to 2 [definitely]); How likely is it that you will eat at least two servings of fruil each day ( -2 [very unlikely] to 2 [very likely]); and How sure are you that you will eat at least two servings of fruit each day ( -2 [surely not] to 2 [surely]). Cronbach's $\alpha$ was .90 and a mean score for intention was computed.

Use, credibility and personal relevance of feedback letter. At $T 2$, the extent to which the feedback had been read (from 0 [not at all] to 3 [completely]), the credibility of the feedback ( 2 [very incredible] to 2 [very credible]) and personal relevance of the feedback were also assessed. Personal relevance was asked with four items on 5-point Likert scales ranging from -2 (totally disagree) to 2 (totally agree), i.e., the information was specifically directed to me, the information was of personal relevance to me, I felt personally addressed, the information was adjusted to my personal situation. 
TTM measures. Data on TTM constructs were collected, but were only used as the diagnostic tool to generate the feedback. In the PCM condition, pros, consciousness-raising, dramatic relief, and environmental re-evaluation process of change were measured. In the CM condition, cons, self-efficacy and the selfreevaluation process of change were assessed. In the AM condition, self-efficacy, and the counterconditioning, helping relationships, reinforcement management, and stimulus control processes of change were assessed. More detailed information about the decisional balance, self-efficacy and processes of change scales can be derived from other studies (see previous chapters; Greene et al., 2004; Ma et al., 2002; Padula et al., 2003)

\section{Data anályses}

After $\mathrm{T} 0$, stage of change was measured again at $\mathrm{T} 1$, before respondents received the tailored feedback to obtain the most accurate indication of stage of change. Respondents who progressed further than the contemplation stage between T0 and T1 were excluded from all further analyses $(n=112)$.

Descriptives and cross-tabulation were used to describe fruit intake, stage of change and demographic characteristics of the remaining 524 respondents. To test whether randomization was successful, analysis of variance and Chi-square tests were used to detect differences between feedback conditions in baseline fruit intake, stage of change, age, sex, level of education and ethnicity. Next, logistic regression analyses were conducted with dropout as the dependent variable and condition, fruit intake, stage of change, age, sex, level of education and ethnicity as the independent variables to test if any of these variables were associated with not completing the entire study.

All analyses were stratified according to stage of change. For fruit intake, a t-test was conducted to test whether fruit intake at T3 was higher than at T1. Condition was dummy-coded into three dichotomous variables. For all outcome measures, $\mathrm{AM}$ versus $\mathrm{PCM}$, and $\mathrm{CM}$ versus $\mathrm{PCM}$ were entered first as independent variables. Next, $A M$ versus $C M$ was included as an independent variable. It was hypothesized that for precontemplators the PCM feedback would reveal higher fruit intake, more positive intentions and more stage progress than the $\mathrm{CM}$ and AM feedback. For contemplators it was hypothesized that the CM feedback would reveal higher fruit intake, more positive intentions and more stage progress than the PCM and AM feedback. To test for these differences in fruit intake at $\mathrm{T} 3$ (with fruit intake at $\mathrm{T} 1$ included as a covariate) and intention at $\mathrm{T} 2$, the effects of the three feedback conditions were analyzed for precontemplation and contemplation separately by means of linear regression. Cohen's standardized differences between means $(d)$ were computed to interpret 
the magnitude of effect size. A small effect size was defined as $d=.20$, a medium effect size as $d=.50$ and a large effect size as $d=.80$ (Cohen, 1988).

Logistic regression analyses were conducted to test for differences between the three feedback conditions on forward stage transition (forward stage transition between $\mathrm{T} 1$ and $\mathrm{T} 2$ coded as 1 and backward or no stage transition coded as (0). Next, this procedure was repeated for progress between T1 and T3. Odds ratios (OR) were computed as the effect size estimate. Though no clear interpretation guidelines for the magnitude of OR exist, an OR near 1.5 or 2.0 is usually interpreted as meaningful (Kraemer, 2004).

Finally, analyses of variance (ANOVA) with Scheffé multiple comparison tests were conducted to test for differences between feedback conditions in the use, credibility and appreciation of the feedback letter. For the ANOVAs, etasquared $\left(\eta^{2}\right)$ was used as the effect size estimate (Clark-Carter, 2003). A small effect size was defined as $\eta^{2}=.01$, a medium effect size as $\eta^{2}=.06$, and a large effect size as $\eta^{2}=14$ (Cohen, 1988). If the F-test was significant, Cohen's $d$ was calculated for the post-hoc tests. All tests were two-tailed and alpha was set at .05

\section{Results}

\section{Participants}

The study sample comprised $336(64 \%)$ men and 188 (36\%) women; mean age was $39.25(\mathrm{SD}=11.42)$ years and ranged from 18 to 74 . Of the respondents, $31 \%, 43 \%$ and $25 \%$ had a low, medium or high level of education, respectively. Most respondents were of Dutch origin $(94 \%)$. Pre-intervention fruit intake averaged $139(\mathrm{SD}=116)$ grams per day, and $87 \%$ of the respondents ate less than the recommended minimum intake levell of 250 grams per day. No differences between the three feedback conditions were found, indicating that the randomization was successful. Further, no differences were found between completers and non-completers in sex, age, level of education, ethnicity, fruit intake and feedback condition, but precontemplators were significantly less likely to complete the study then contemplators $(13 \%$ vs. $7 \%$; OR $=1.88 ; p=.04)$.

\section{Effects of feedback conditions on fruit intake}

Stratified analysis by stage of change showed no difference in fruit intake between $\mathrm{T} 1$ and $\mathrm{T} 3$ in fruit intake for precontemplators $(t=1.07, p=.29, d=.07)$, while for contemplators fruit intake increased significantly between T1 and T3 $(t=$ 
$-3.42, p=001, d=.22$ ). For precontemplators; a small effect approaching significance for $\mathrm{AM}$ versus PCM (beta $=.12, p=.07$ ) was found, indicating that precontemplators who received the action-matched intervention, ate more fruit than precontemplators who received the precontemplation-matched intervention. For contemplators, no significant effects of condition on fruit intake were found (see Tables 6.1 and 6.2).

Table 6.1 Pre- and posttest fruit intake by feedback condition and stage at T1

\begin{tabular}{|c|c|c|c|c|c|c|}
\hline \multirow[b]{2}{*}{ Fruit intake (gr/day) } & \multicolumn{2}{|c|}{ PC-matchied } & \multicolumn{2}{|c|}{ C-matched } & \multicolumn{2}{|c|}{ A-matched } \\
\hline & T1 & T3 & T1 & T3 & TI & T3 \\
\hline Precontemplation & $\mathrm{n}=90$ & $\mathrm{n}=80$ & $\mathrm{n}=98$ & $\mathrm{n}=79$ & $n=101$ & $n=93$ \\
\hline \multirow{5}{*}{ Contemplation } & 117.87 & 100.55 & 132.92 & 117.99 & 126.13 & 129.57 \\
\hline & 86.81 & 87.67 & 137.70 & 90.90 & 116.49 & 109.68 \\
\hline & $\mathrm{n}=77$ & $n=73$ & $\mathrm{n}=82$ & $\mathrm{n}=76$ & $n=76$ & $n=69$ \\
\hline & 167.72 & 191.94 & 156.84 & 178.72 & 140.65 & 185.03 \\
\hline & 135.24 & 128.87 & 106.25 & 110.76 & 100.25 & 110.01 \\
\hline
\end{tabular}

Note. $\mathrm{M}=$ mean, $\mathrm{SD}=$ standard deviation

\section{Effects of feedback conditions on intention}

For contemplators, a small to moderate effect on intention was found for the PCM feedback compared to the CM feedback, indicating that contemplators in the precontemplation-matched interventions had a significantly more positive intention at T2 than contemplators in the contemplation-matched condition (beta $=-0.27, p=.02$; Table 6.2). Further, no differences in intention were found between the action-matched and precontemplation-matched, or between the actionmatched and the contemplation-match for the contemplators. For precontemplators no effects of the feedback conditions on intention were found (Table 6.2).

\section{Effects of feedback conditions on stage progress}

\section{Between T1 and T2}

No effects of the feedback conditions on forward stage transition between $\mathrm{T} 1$ and $\mathrm{T} 2$ were found (Table 6.3). Of the respondents in precontemplation at $\mathrm{T} 1,19 \%$, $19 \%$, and $18 \%$ were in a more advanced stage of change immediately after the feedback, for PCM, CM and AM feedback condition, respectively. Progress to a more advanced stage occurred in $17 \%, 27 \%$, and $18 \%$ of the contemplators for the three feedback conditions, respectively. 
Table 6.2 Effects of feedback conditions on fruit intake and intentions

$\begin{array}{lllllllll}\text { Fruit intake at T3 } & \text { B } & \text { SE B } & \text { B } & \text { A } & \text { B } & \text { SE B } & \beta & \text { d } \\ \text { AM versus PCM } & 23.54 & 12.89 & .12^{\text {a }} & .17 & 3.65 & 16.50 & .02 & .10 \\ \text { CM versus PCM } & 15.34 & 13.40 & .07 & .13 & -10.18 & 16.07 & -.04 & .06 \\ \text { AM versus CM } & 8.19 & 12.93 & .04 & .04 & 13.83 & 16.32 & .06 & .20\end{array}$

\section{Intention at $\mathrm{T}_{2}$}

\begin{tabular}{lllllllll} 
AM versus PCM & 0.07 & 0.13 & .04 & .08 & -0.11 & 0.11 & -0.07 & .16 \\
CM versus PCM & 0.99 & 0.13 & .05 & .11 & -0.27 & 0.11 & $-0.18^{*}$ & .37 \\
AM versus CM & -0.10 & 0.13 & -.02 & .04 & 0.16 & 0.11 & 0.11 & .23 \\
\hline
\end{tabular}

Note. ${ }^{a} p=.07 ;{ }_{r}^{* *} p=.02 ; d=$ Cohen's $d$ standardized differences between means

\section{Between T1 and T3}

No effects of the feedback conditions on forward stage transition between T1 and $\mathrm{T} 3$ were found (Table 6.3). Of the respondents in precontemplation at $\mathrm{T} 1,21 \%$, $17 \%$, and $22 \%$ were in a more advanced stage of change one week after the feedback for the PCM, CM and AM feedback, respectively. Progress to a more advanced stage occurred in $32 \%, 30 \%$, and $38 \%$ of the contemplators at T1 for the three feedback conditions, respectively.

Table 6.3 Effects of feedback conditions on stage progress

\begin{tabular}{lcccc}
\hline & \multicolumn{2}{c}{ Precontemplation } & \multicolumn{2}{c}{ Contemplation } \\
Progress T1-T2 & OR & $95 \% \mathrm{Cl}$ & OR & $95 \% \mathrm{CI}$ \\
\cline { 2 - 4 } AM versus PCM & 0.93 & $0.45-1.94$ & 1.11 & $0.48-2.55$ \\
CM versus PCM & 1.03 & $0.50-2.14$ & 1.81 & $0.84-3.90$ \\
AM versus CM & 0.90 & $0.44-1.84$ & 0.62 & $0.44-1.84$ \\
Progress T1-T3 & & & & \\
AM versus PCM & 1.02 & $0.49-2.11$ & 1.31 & $0.66-2.63$ \\
CM versus PCM & 0.73 & $0.33-1.63$ & 0.94 & $0.47-1.89$ \\
AM versus CM & 1.39 & $0.64-3.02$ & 1.39 & $0.53-2.12$ \\
\hline
\end{tabular}




\section{Use, credibility and personal relevance of feedback}

The feedback letter was largely or completely read by $74 \%$ of the precontemplators and by $79 \%$ of the contemplators. ANOVA revealed no differences in the extent to which the feedback letter was read between the PCM, CM and AM feedback conditions for precontemplators (F $(2,289)=0.90, p=.41, \eta^{2}$ $=.01)$ or contemplators $\left(\mathrm{F}(2,235)=0.76, p=.47, \eta^{2}=.01\right)$. The feedback letter was rated as credible or very credible by $64 \%$ of the precontemplators and by $80 \%$ of the contemplators.

ANOVA revealed no differences in credibility between the PCM, CM and AM feedback conditions for precontemplators $\left(\mathrm{F}(2,289)=0.43, p=.65, \eta^{2}<.01\right)$ or contemplators $\left(\mathrm{F}(2,235)=0.32, p=.73, \eta^{2}<.01\right)$.

For precontemplators, ANOVA revealed no differences between the feedback conditions in the four evaluation items on personal relevance (see Table 6.4). For contemplators, ANOVA showed that contemplators perceived the action-matched feedback as more personally relevant than the contemplationmatched $(d=.43, p=.03)$ and precontemplation-matched feedback $(d=.67, p<$ .001). Contemplators further evaluated the action-matched feedback as more adjusted to their personal situation than the precontemplation-matched feedback $(d=.42, p=.05)$, but not more than the contemplation-matched feedback $(d=.22$, $p=.42)$.

\section{Discussion}

The present study failed to support the superiority of stage-matching compared to stage-mismatching for fruit intake. Precontemplators receiving the actionmatched feedback had marginally significant higher fruit intake compared to those in the precontemplation-matched condition, but no differences between the conditions were found in intention, stage progression, use or personal relevance of the feedback. Contemplators in the precontemplation-matched condition had higher intentions than contemplators in the contemplation-matched condition, but rated the action-matched feedback as most personally relevant and most adjusted to their personal situation. No differences between the conditions were found for fruit intake or stage progression for contemplators.

Several explanations can be given for the inconsistent results. First, stagematched interventions can only be more effective than mismatched interventions in increasing motivation and behavior change if the factors targeted in stagematched interventions are indeed stage-specific. The TTM postulates that this is the case, but earlier studies to test TTM constructs and fruit intake indicated that self-efficacy and specific processes of change may predict different stage transitions. 


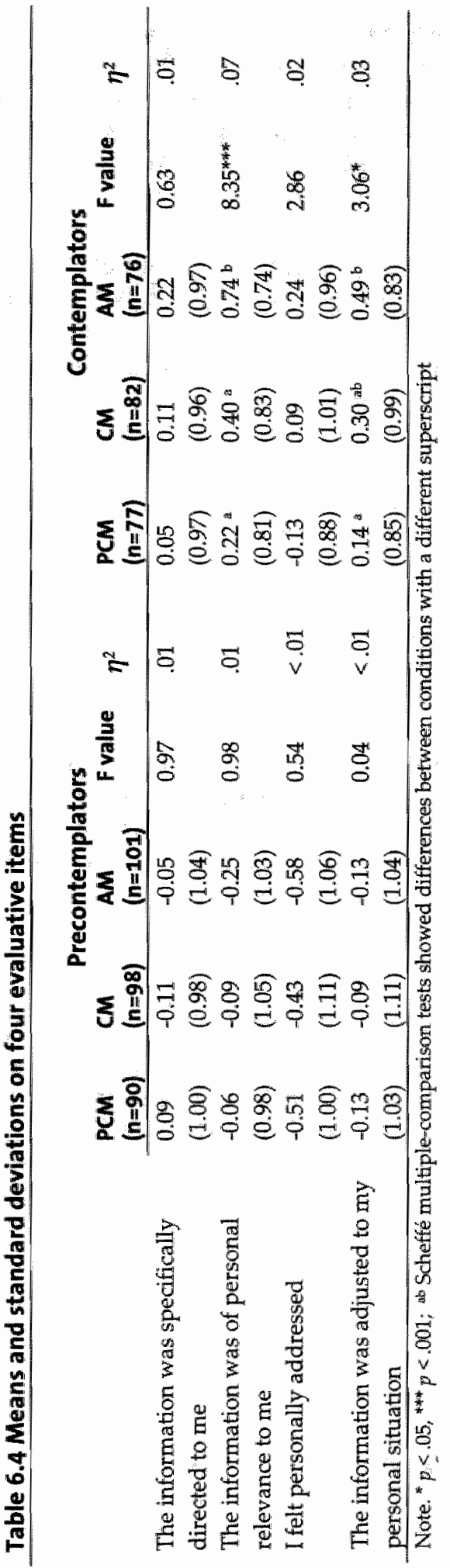


For instance, self-efficacy has been shown to predict both forward stage transition out of precontemplation and contemplation (chapter 2; De Vet, De Nooijer et al., 2005a), and the behavioral processes supposedly relevant for action, such as stimulus control and counterconditioning were also associated with stage transition from precontemplation and contemplation (chapter 3; De Vet et al., submitted). It has been argued that such a lack of stage-specifity of stage transition determinants could indicate the existence of pseudostages. Pseudostages reflect a stage-like categorization of a continuous variable, for example intention, rather than true stages (Sutton, 2000a; Weinstein et al., 1998). Transtheoretical stages of change based on a continuous variable would make stage-matching irrelevant, since respondents in different (pseudo)stages will profit from the same intervention.

Second, the present feedback conditions were stage-matched on only two aspects of health behavior change interventions, i.e., content and communication. As Dijkstra et al. (1998) discussed, also other intervention components might require an adaptation to stage of change, such as the method of intervention (e.g., mass-media or interpersonal), the intervention channel (e.g.r website or a print leaflet), or the message source (e.g., peers or experts). To our knowledge, no match-mismatch tests have been conducted that matched more aspects to stages of change than content and communication.

It should be noted that the feedback conditions had different effects for precontemplators than for contemplators. Since people in different stages may benefit from different interventions, the result may be considered a sign of the existence of stages of change. However, the effects are not in line with predictions made based on the TTM, since the stage-mismatched feedback outperformed the stage-matched feedback in both stages and the results do not support the applicability of the TTM in promoting fruit intake.

The mismatched feedback had some value in two situations. First, we found some indications that in precontemplators AM feedback had more effect on fruit intake than PCM feedback. An explanation for the positive effects of AM feedback in precontemplators might be carried back to the classification of the stages of change for fruit intake. If someone increased fruit intake recently from none to one daily serving of fruit, s/he may not intend to increase fruit intake further. Since this person eats less fruit than recommended and is not motivated to start eating according to recommended intake levels, she will be classified as precontemplator. However, s/he recently made changes and might have applied behavioral processes and strategies to achieve the change. So, one could also consider this person to be in the action stage. It has been argued that defining stages of change for dietary behaviors is different than defining stages for smoking and other behaviors for which complete cessation is the goal (Lamb \& Joshi, 2004; Ni Mhurchu et al., 1997; Povey et al., 1999).

Second, in contemplators the PCM feedback condition had a small to moderate effect on intention. In line with TTM propositions, contemplators generally show high scores on precontemplation-related factors such as pros, and the consciousness-raising, dramatic relief, and environmental re-evaluation processes of change, since contemplators are assumed to have already passed the 
precontemplation stage. In the individualized feedback, such positive baseline scores generated feedback messages with a positive and reinforcing content. As outlined in the Social Learning Theory, positive reinforcement can provide a powerful mechanism to change (Bandura, 1977).

Due to limitations of the study, the results should be interpreted with some caution. We did not include a no-feedback control group, since the main abjective was to test whether stage-matched feedback would outperform the stage-mismatched feedback and not whether providing (stage-matched) feedback would perform better than no feedback at all. Further, the use of an Internet research panel may have reduced the external validity of the results. Our sample comprised a large proportion of males $(63 \%)$, while samples in dietary research often comprise a majority of females (Greene et al., 2004; Horacek et al., 2002; Kristal, Hedderson, Patterson, \& Neuhauser, 2001). Past research has shown that males are more likely to be in early stages and it has been argued that public health should take an effort to reach men and unmotivated groups (Kristal et al., 2001; Laforge, Greene, \& Prochaska, 1994; Vallis et al., 2003). The use of an Internet panel provided this opportunity. A further advantage of the Internet panel was that immediate feedback could be given, so that the feedback was as accurate as possible. It, therefore, allowed for controlling stage-instability that could have caused unplanned mismatching for respondents who progressed to preparation, action and maintenance between $\mathrm{T} 0$ and $\mathrm{T} 1$.

To conclude, consistent with earlier studies on stage-matched and mismatched interventions (Dijkstra et al., 1998; Quinlan \& McCaul, 2000), our results do not provide evidence for the merits of matching interventions to stages of change for fruit intake as defined by the TTM. 


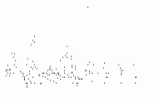

the

2 


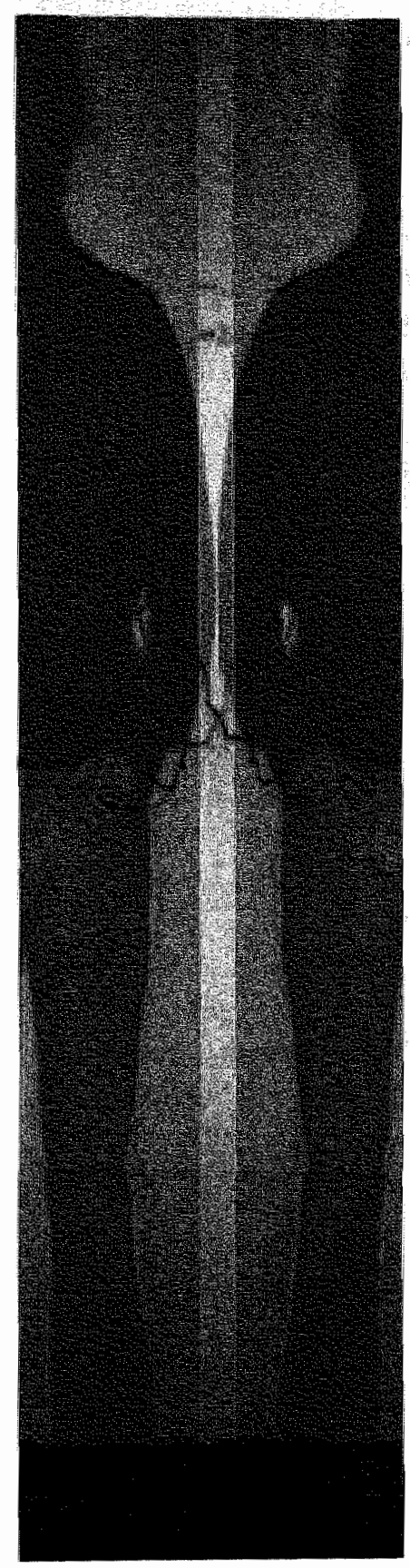

\section{General Discussion}


3

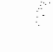

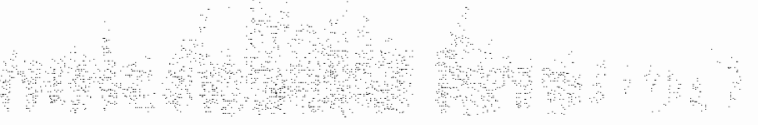




\section{General discussion}

The present thesis deals with testing assumptions and predictions of the Transtheoretical model of behavior change (TTM). Three studies were conducted. First $t_{s}$ a three-round Delphi study was executed to achieve an integrative overview of stage transition determinants derived from a multiple-theory perspective, based on knowledge and experiences from experts in the field (chapter 1). Next, a Dutch adult cohort was assessed six times with varying time intervals using electronic questionnaires. The objectives in this longitudinal study were threefold, 1) to gain insight into predictors of stage transitions (chapters 2 and 3), 2) to examine the stability and changeability of stages of change with a focus on patterns of change and specific sequences within these patterns (chapter 4), and 3) to explore whether pre-action stages of change reflected pseudostages, i.e, a stage-like categorization of a continuous behavioral intention (chapter 5). The third study comprised a randomized trial in which we tested whether individualized web-based stage-matched feedback induced more stage progress, more positive intentions to eat ample amounts of fruit and higher fruit intakes than stage-mismatched feedback (chapter 6).

The purpose of this final chapter is to summarize and integrate the findings from the studies presented in this thesis. The main results are discussed separately for part 1 on predictors of stage transitions (chapters 1, 2, and 3) and part 2 on staging health behavior change (chapters 4,5 , and 6). Further, methodological and theoretical issues will be discussed, as well as implications for future research and practice. 


\section{Part 1 Predictors of stage transitions}

The Delphi study achieved generally high consensus among experts on various stage transition determinants, although experts agreed to a lesser extent about determinants of stage transitions from precontemplation to preparation (chapter 1). It might be that these stage transitions are more complex, but the finding might also reflect the relative lack of knowledge about how to motivate persons and the strong emphasis in health education (and other domains of behavioral change) on action-initiating interventions. Behavior change has long been considered a two-step process, from unhealthy to healthy behavior (Prochaska \& Velicer, 1997a). Still, most attention goes to individuals who express an intention to change their unhealthy behaviors, even though only a small proportion of a population may be ready for change (Laforge et al., 1999; Nigg et al., 1999). The focus on people who are ready to change their behavior might also stem from pragmatic motives. Individuals unmotivated to adapt their lifestyles are probably a lot more difficult to include in research and practice on health behavior change (Prochaska \& Velicer, 1997b). Consequently, little is known about stage transition determinants for these early stages. In chapters 2 and 3 , we explicitly focused on predictors of forward transition from precontemplation and contemplation, and the cross-sectional correlates of these stages. In the next subsections, the differences between cross-sectional and longitudinal results will be discussed first, followed by a reflection on the difficulties with the identification of stagespecific predictors of stage transitions. Finally, the importance of the specific TTM components, i.e., decisional balance, self-efficacy, and processes of change as applied to fruit intake will be discussed.

\section{Cross-sectional correlates of stages of change versus longitudinal predictors of stage transitions}

To date, most research examining differences in psychosocial variables across the stages of change used cross-sectional designs (see for example Fontes de Oliveira et al., 2005; Greene et al., 2004; Kloek et al., 2004; Lam, Chan, Ho, \& Chan, 2004). Often it is assumed that if a significant difference in a variable (e.g., pros or cons) between stages is found, the variable is likely to predict stage transitions. However, cross-sectional association is not the same as prediction or determination. It might as well be that the variable is influenced by a stage transition, or that a third, unknown, variable influences both stage of change and the particular psychosocial variable (Sutton, 2000b). To detect stage model indications from cross-sectional research, not only should one find significant differences in a psychosocial variable between the stages of change, but the 
differences should also show a non-linear (discontinuous) pattern across the stages. More specifically, the increase of the variable should not be consistently incremental (or decremental) (see Figure 1, cf, Sutton, 2000b). To exemplify, pros should increase significantly between precontemplation and contemplation, but should not differ significantly between contemplation and preparation. Frequently, cross-sectional research showed a pattern with a small, but significant increase between two stages (e.g., between precontemplation and contemplation), and a significantly larger increase between two other stages, for example between contemplation and preparation (e.g., chapter 2 and 3, and for example Campbell et al., 1998; Ma et al., 2002). Such a pattern is often considered a discontinuity pattern, while the variable actually increases between three stages of change (see Figure 1). This pattern would also confirm a linear model instead of discrete stages (Sutton, 2000b).

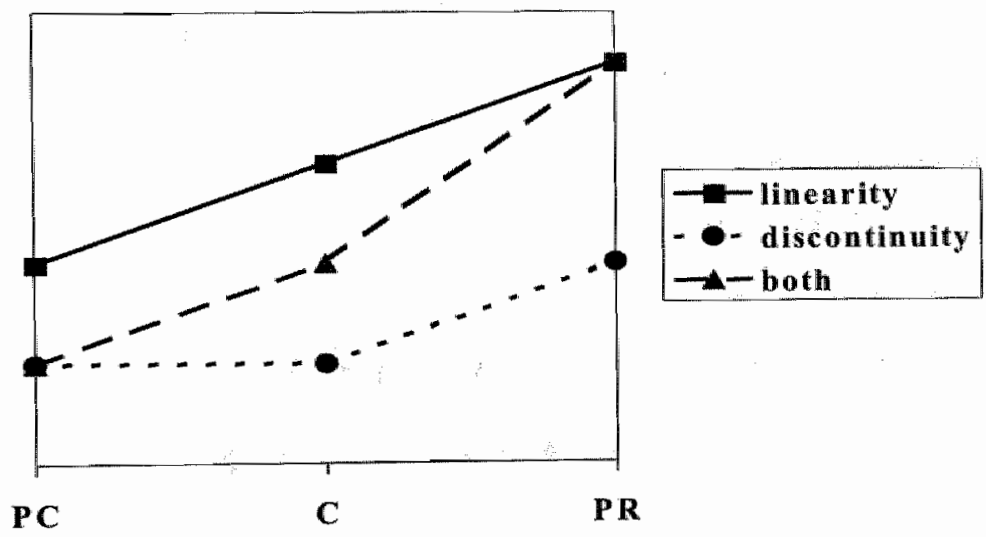

Figure D.1. Crass-sectional patterns across stages of change

In chapters 2 and 3 we analyzed differences in decisional balance, selfefficacy and processes of change across stages of change using cross-sectional methods as well as predictions of stage transitions by the same variables using longitudinal methods. The results showed that the longitudinal results differed from the cross-sectional results in two important aspects. First, cross-sectional differences in particular variables were found, while those variables did not predict transition between these stages longitudinally. For instance, respondents in preparation used more experiential processes than respondents in the contemplation stage of change, but experiential processes did not predict forward stage transition out of contemplation. Second, for some variables, no cross- 
sectional differences were found, while those particular variables did predict stage transition. For example, respondents in preparation and contemplation did not differ significantly from respondents in the preceding stage of change with respect to self-efficacy in the cross-sectional analyses; while self-efficacy did predict forward stage transition out of both precontemplation and contemplation in the longitudinal analysis.

Generally, prospective designs allow for stronger inferences than crosssectional designs, since the possibility that the increase or decrease in a psychosocial variable is a consequence of stage progression can be ruled out (Kraemer, Wilson, Fairburn, \& Agras, 2002). However, prospective designs cannot provide proof of causality, since mere prediction does not allow for identifying causal paths and causal processes. For instance, we found a higher frequency of forward stage transition amongst precontemplators who see many advantages of the target behavior than amongst precontemplators who see few advantages of the target behavior. So, pros tell us who is more likely to make a stage transition (prediction); this finding does not tell us why and how this stage transition occurs (explanation). Although prediction is a prerequisite for explanation, a predictor of change does not necessarily explain (the process of) change (Sutton, 1998). To conclude, our results seem to confirm that correlates of a particular stage differ from predictors of change (cf., Brug, Oenema et al., 2005).

\section{Stage-specific predictors of stage transitions}

In the first studies of this thesis, described in part one, no straightforward stagespecific predictors of stage transitions could be identified. In the Delphi study (chapter 1), stages of change expert respondents agreed on various transition determinants, but repeatedly these factors were assumed to predict more than one stage transition. For instance, social support, self-efficacy and (perceived) control-related issues were considered important for all stage transitions between contemplation and maintenance. The study described in chapter 2 has shown that self-efficacy predicted forward stage transitions out of both precontemplation and contemplation. Further, chapter 3 revealed that behavioral processes predicted these stage transitions as well, and additionally transition into the action stage. Experiential processes predicted forward stage transition out of precontemplation, and transition into action.

From the literature it remains unclear what results would indicate that stages of change are qualitatively different. In the literature, three perspectives on this stage model assumption can be found. First, as phrased by Weinstein and colleagues, "to justify calling health behavior a stage process, some barriers must be more important at certain stages than others [...]. Other factors, however, might facilitate progress regardless of stage" (Weinstein et al, 1998, p. 291). To 
confirm this first perspective, predictors of stage transition should be evaluated in terms of the magnitude of the relationship between a predictor and a stage transition, i.e., in terms of effect sizes. The results described in chapter 3 indeed showed that the behavioral processes predicted transition into the action stage better (Odds ratio $=3.47$ ) than the transition out of precontemplation (Odds ratio $=2.56$ ) and contemplation (Odds ratio $=2.62$ ).

Second, Sutton stated that a stage process would be indicated if "the set of factors that influence stage transition from stage I to stage II is different from the set of factors that influence stage transition form stage II to stage III " (Sutton, 2001, p. 181). He also acknowledged that there may be overlap in these sets, since some variables may be important at every stage. To confirm this second perspective, analyses should be conducted to evaluate whether or not the variables contribute to the prediction of stage transitions. Chapters 2 and 3 showed that the predictors of stage transitions indeed do appear to form different sets. Pros, self-efficacy, behavioral and experiential processes constituted the relevant set of predictors of progress out of precontemplation. The set of predictors of progress out of contemplation consisted of self-efficacy and behavioral processes. Stage-matched interventions usually target several concepts simultaneously. It can, therefore, be useful to look at sets of predictors instead of studying one predictor at the time. However, taking into account the large overlap between the sets we found, successful matching of these interventions to stages would be difficult.

Courneya and colleagues proposed a third perspective on the issue of stage specificity by stating that "stage models are supported not only if they identify different determinants for each stage transition, but also if they identify different interventions for each stage transition even if the interventions target the same determinant" (Courneya et al., 2001, p.146). For example, social cognitive theory describes several strategies for enhancing self-efficacy, such as mastery experience, modeling, and persuasion (Bandura, 1986, 1998). As suggested by Armitage, Povey and Arden (2003), it might be that modeling will increase selfefficacy for precontemplators, while mastery experiences will produce best results for preparators. To date, no studies have tested this stage model perspective.

We propose a fourth perspective, i.e., that the beliefs that constitute a stage transition determinant might be stage-specific. For example, the I-Change model makes a distinction between emotional and rational pros and cons (De Vries et al., 2003). With respect to fruit intake, a rational con might refer to preservation of fresh fruits, and an emotional con could concern worries about chemicals on fruit. In some stages, for example, emotional cons might be more important than rational cons and vice versa for other stages. However, no evidence from experimental or longitudinal studies exists to date for this perspective (chapter 1). 
Experimental studies that test rational and emotional beliefs, the proposed determinants of change (isolated or in sets) or the strategies towards change can provide a more definite view on the qualitative difference of stages of change. Yet, this would be a time-consuming effort due to the almost unlimited combination of beliefs, predictors, sets and strategies that might be of influence. To summarize so far, presumed separate predictors of stage transition do not seem to be stage-specific, and therefore the TTM may not be distinct from other (continuum) models of behavior change.

\section{Pros, cons, self-efficacy, and processes of change}

Although the studies presented in this thesis do not provide clear evidence for qualitative different stages of change, most TTM components (pros, self-efficacy, experiential and behavioral processes) did predict stage transitions for fruit intake.

The TTM assumes that in early stage transitions, pros and experiential processes would be of importance, while self-efficacy and behavioral processes would gain relevance once respondents are ready for change (Prochaska, DiClemente, \& Norcross, 1992; Prochaska et al., 2003). Our results showed that for fruit intake self-efficacy and behavioral processes might already be relevant for progress out of precontemplation. Two explanations can be given. First, as previously discussed in chapter 2 and 3 , respondents progressing from precontemplation may already have made small behavior changes, which did not make them comply to recommended intake levels yet. Such small changes in fruit intake may of course very well require engagement in behavioral processes and strategies to achieve the change. So, this is related to the continuous dependent variable, i.e., fruit intake. Second, Prochaska and colleagues (2002) argued that individuals may be in precontemplation for two reasons, namely because 1) they are under-informed or uninformed about the consequences of their behavior, or 2) they may have become demoralized about their ability to change due to failures in previous change attempts, i.e., have low self-efficacy expectations. If people are in precontemplation for the first reason, a cognitive focus (raising awareness, emphasizing pros etc.) might indeed determine progress to further stages. If individuals are in the precontemplation stage because of failures in previous attempts, a behavioral focus (increase self-efficacy, practice skills etc.) might lead to progress to more advanced stages of change.

Finally, in chapter 3 two comments have been made about the processes of change. First, to justify the term 'processes of change', effects of actual use of these so-called processes should preferably be tested in experimental research. The questionnaire items normally used, also in the present thesis, to assess the experiential processes do not assess actual use, but are much like belief items. 
Second, according to the TTM, processes of change should be assessed retrospectively. However, recalling the use of processes of change may not be accurate. Some people may not be aware of having applied particular processes in the past; while people who are more involved in the issue of fruit intake (e.g., respondents who progress to action) might be more able to recall the processes of change applied. Additionally, in line with the second comment, doubts have been raised about the extent to which individuals are actually able to report on internal processes. Such processes may occur mainly at the non-conscious level (Nisbett \& Wilson, 1977).

With the exception of cons (as discussed in chapter 2, cons did not predict stage transitions) and despite these points of criticism, the fact that most TTM components predict stage transitions indicates that these components may be useful in understanding behavior change. Opportunities may exist for targeting these components in behavior change interventions.

\section{Part 2 Staging health behavior change}

The question whether behavior change is best described from a staging or a continuum perspective guided the three chapters in part 2 . Chapter 4 examined the extent to which individuals make stage transitions and the pattern and sequence in which people progress and regress through the stages. In chapter 5 , a comparison between stages and pseudostages was made. In chapter 6 , it was tested whether feedback matched to an individual's stage was more effective than feedback mismatched to an individual's stage (i.e., matched to an inappropriate stage).

\section{Stability and changeability of stages of change}

Prochaska portrayed the stages as "a stable quality like traits in that they tend to endure over relatively long periods of time" (Prochaska, 2000, p. 109). He argues that contrary to human traits, which are not particularly vulnerable to change, stages are to some extent dynamic in nature (thus open to change). However, change would not occur easily and special efforts such as interventions would be needed. The length of time an individual spends in a stage may vary, and there is no minimum to the length of time individuals spend in a certain stage. If all factors that are needed to move to a further stage in the sequence are present, people may pass through the stages in a few moments (Norman et al., 1998; Prochaska, DiClemente, \& Norcross, 1992; Weinstein et al., 1998). 
The results in chapter 4 showed that stages of change were rather stable, although short-term instability also occurred. Instability mostly involved transitions to an adjacent stage of change, and in accordance with the TTM it appeared that stage-skipping is not prevalent in stage progression. However, an important assumption of studying stage sequences is that stage transitions are not missed in measurement (Sutton, 2001). Stage transitions might be missed, since according to the TTM, some individuals may pass a stage very quickly; it may even be a split second (Kremers, Mudde, \& De Vries, 2004). As a consequence, the result that respondents mostly progress and regress to an adjacent stage does not exclude that stage transitions may have occurred already between two assessments. Even respondents who remain in a particular stage for two assessments, may have made transitions in-between assessments.

Further, stage transitions were prevalent even without a planned intervention. One might argue that such "spontaneous" stage transitions result from an invalid stage assessment (chapter 4). The changes in fruit intake, however, largely followed the pattern of stage transitions, which might indicate validity of the stage of change instrument. The validity of the staging instrument should further be demonstrated by showing that the transition profiles result from similar profiles in predictors of stage transitions, such as decisional balance, self-efficacy and processes of change. More specifically, it would be interesting to analyze whether patterns of changes in these cognitions precede the patterns of changes in stages (i.e., transition profiles).

\section{Pseudostages}

The finding in the present thesis that people (by and large) progress and regress through the stages as outlined by the TTM is in line with an essential stage model requirement. Although it is essential requirement, it is not a sufficient one (Weinstein et al., 1998). In other words, meeting this requirement does not prove that behavior change is indeed a staged process. It may still be that the different stages are in fact a behavior change continuum divided into five categories, i.e., pseudostages. When individuals slide forward and backward along that behavior change continuum, the stage model requirement would still be met.

Some support was found for this pseudostages hypothesis. Several indications were presented in chapter 5 that Transtheoretical pre-action stages of change represent a categorization of a continuous intention measure instead of discrete stages. First, strong correlations were found between stages of change and a pseudostage variable. Second, for a majority of respondents, stability and transitions over time in stages of change resembled transitions in pseudostages, while test-retest reliabilities for both measures were approximately similar. Further, indications were found for multicollinearity between stages of change 
and pseudostages. Stages of change and pseudostages may thus reflect the same concept.

The evidence for the lack of stage-specific predictors presented in the first part of the thesis is a further indication that the early stages distinguished in the TTM reflect pseudostages. Furthermore, as we will discuss in the next subsection, the results of the match-mismatch test described in chapter 6 of this thesis provides further support for the hypothesis that stages of change are a categorization of an underlying continuous variable.

\section{Matched and mismatched feedback}

The results described in chapter 6 showed that individualized stage-matched feedback did mot outperform the individualized stage-mismatched feedback in precontemplators and contemplators. This may not be surprising, since we were not able to identify stage-specific transition determinants (part 1). Furthermore, earlier research on stage-matched and mismatched interventions based on the TTM also failed to show that matched interventions were superior (Blissmer \& McAuley, 2002; Dijkstra et al., 1998; Quinlan \& McCaul, 2000). In chapter 6, several explanations for the non-effects of the match-mismatch test were discussed in detail, which will not be repeated in this section. Some other issues that are involved with match-mismatch tests, however, deserve further elaboration. In this subsection, we will discuss the impact of multiple-component interventions on the success of matching interventions to stage of change, followed by an argument on the hypothesized causal chain. Finally, we discuss whether a match-mismatch test is actually a good test to provide evidence in favor or against stages of change.

First, stage-matched interventions generally target multiple concepts simultaneously. However, if one of the concepts actually is irrelevant to individuals in a particular stage of change, the entire intervention may fail to induce significant improvements. For example, the contemplation-matched feedback was the least effective of the three feedback conditions. Consistent with the TTM, the contemplation-feedback targeted cons of fruit intake. However, the cons was the only TTM component not predictive of any stage transitions for fruit intake in the longitudinal cohort study presented in this thesis. Even if targeting self-efficacy or self-reevaluation in contemplators might have been effective, such effects could have been suppressed by irrelevant information on cons. Experimental tests comparing the effects of single component interventions to effects of multiple component interventions can provide more insight into this issue.

It should be considered in what way components, such as pros, cons, or self-efficacy cause intervention effects. Two types of carisality must be taken into 
account (Maclure, 1998). High self-efficacy, for instance, might be a necessary cause for stage transition, which means that stage transition cannot occur without high self-efficacy. This is the first causal statement, i.e., if $Y$ cannot occur without $X, X$ is a necessary cause of $Y$ (Maclure, 1998). It might also be that high selfefficacy will always result in stage transition, but that transition is not necessarily caused by high self-efficacy. This is the second causal statement, i.e., if $X$ always results in $Y$, then $X$ is a sufficient cause (Maclure, 1998). Thus, if a single component reveals no intervention effects, it might still reveal effects if the component is targeted simultaneously with another component. Furthermore, the effectiveness of a particular intervention might be maximized at a particular level of one of the components or might even onlly be detected if one of the components reaches a certain threshold. In this area an interesting test based on Social Cognitive Theory was conducted by Dijkstra and De Vries (2001). Individuals randomly received outcome expectations information, self-efficacy information, or both types of information. Results showed that targeting outcome expectations led to positive changes in outcome expectations and in self-efficacy, while targeting self-efficacy only led to an increase in self-efficacy. Targeting both selfefficacy and outcome expectations did not reveal better results than the outcome information only. This implies that more is not necessarily better, since targeting multiple components did not outperform the single component information.

The second point to be discussed relates to the proposed causal chain that should result in behavior change. The TTM proposes that a change, for example, in pros should result in stage transition, and stage transition in turn will leadl to behavior change. According to the TTM, actual behavior change thus may take a while for some individuals, especially for those initially in the early stages of change (Prochaska \& Velicer, 1997a). However, the results described in chapter 6 showed a small effect on behavior change, but not on intentions or stage transition, and remarkably only for precontemplators receiving action-matched feedback. In conclusion, when developing and evaluating stage-matched interventions not only should we test whether or not an intervention has effects, but efforts should also be taken to gain understanding into how, when, why and for whom an intervention works (cf., Hardeman et al., 2002; Michie \& Abraham, 2004).

Finally, it is debatable whether a match-mismatch test is actually a good test to provide evidence in favor or against the validity of stages of change. On the one hand, a match-mismatch test that shows no superior effects of the matched condition does not necessarily provide evidence against the validity of stages of change. Based on the results described in chapter 6, we can only conclude that for precontemplators, targeting precontemplation-relevant factors as proposed by the TTM might be less effective than addressing the factors the TTM specifies as relevant for action in fruit intake. For contemplators, it seems that targeting contemplation-specific factors might not be as useful for fruit intake 
as one may expect from the TTM. We ought to acknowledge that the weak effects of the feedback may also be attributable to a failure in designing an effective intervention in general. However, we did apply an intervention technique, i.e., individualized computer-tailoring, that has shown promise in the past also for dietary behaviors (Brug et al., 2003; Kroeze, Werkman, \& Brug, in press). On the other hand, a successful match-mismatch test does not definitely prove a staging process either. The stage model requirement that matched interventions are more effective than mismatched interventions should be analyzed by testing the interaction between stage of change and intervention condition (Weinstein et al., 1998). Remarkably, similar analyses have been conducted in research on continuum models of behavior change, i.e., by examining the intention-behavior relationship in the Theory of Planned Behavior (Sheeran, 2002; Sheeran \& Abraham, 2003). In predicting behavior, the interaction between intention and condition or a particular variable (e.g.x anticipated regret or implementation intentions) is included in a moderator analysis (Abrahan \& Sheeran, 2003; Sheeran, Webb, \& Gollwitzer, 2005). If a moderator is thus identified, this moderating variable is often interpreted as strengthening or weakening the intention-behavior relationship, i.e., in terms of bridging or creating the intentionbehavior gap. For example, in an experimental study, anticipated regret (expectations of negative feelings after a behavioral action or decision) was manipulated. The results showed that intentions better predicted behavior for the anticipated regret group than for the control group (Abraham \& Sheeran, 2003). Such moderators are often treated as strategies that can be useful to help people to act on their positive intentions, so-called volitional strategies (Milne, Orbell, \& Sheeran, 2002). Two remarks can be made: First, moderators of intention-behavior relationships are only volitional strategies provided that the experimental condition outperforms the control condition for a positive intention value. If the experimental manipulation (e.g., anticipated regret) also reveals better results for people with no intention to change, the tested strategy might as well act motivationally. As a consequence, not only should one evaluate whether or not a strategy improves intention-behavior consistency, but also for whom the intention-behavior relationship becomes more consistent. Second, examining the interaction between stage of change and experimental condition actually seems not a very strong test of the existence of stages. As argued in chapter 5 , the precontemplation stage might reflect an unmotivated pseudostage (neutral or negative continuous intention score), whereas contemplation/ preparation might reflect a motivated pseudostage (positive continuous intention score; see the $x$ axis in Figure 2). The hypothetical match-mismatch test in Figure 2 shows a significant interaction between condition (precontemplation-matched or contemplation-matched) and stages of change that might reflect pseudostages. This successful match-mismatch would be significant for both measures, stages of 
change and continuous behavioxal intention, and would therefore not provide convincing evidence for the existence of stages of change.

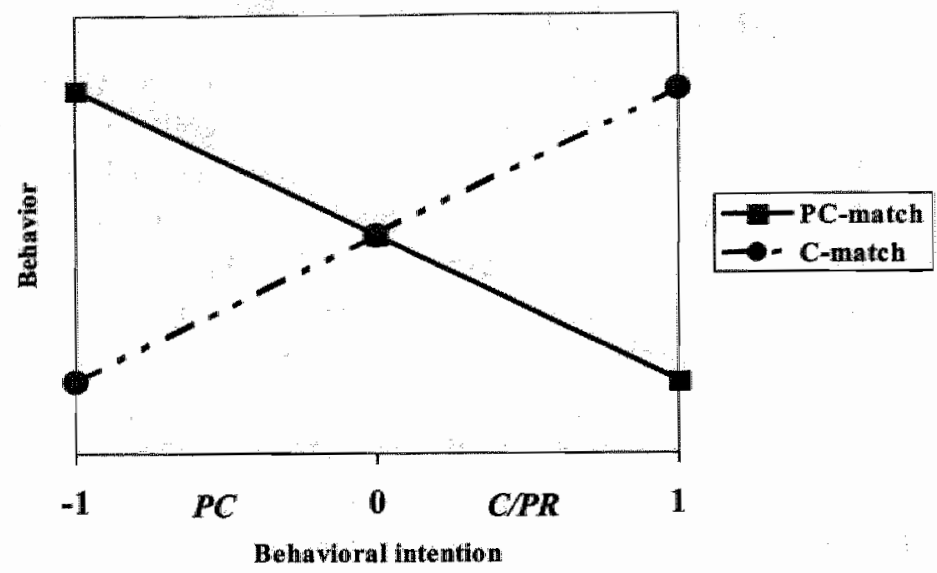

Figure D.2. Successful match-mismatch test for (pseudo)stages

\section{Methodological issues}

The results and conclusions should be interpreted in the light of the potential methodological strengths and limitations to the validity of the research. First, the samples used in the studies, the study designs employed, the measurement instruments used and the statistical analyses conducted will be discussed. Next, the strengths and weaknesses of collecting data and delivering feedback via the Internet will be examined.

\section{Samples}

Two kinds of samples were used in the studies described in this thesis. The first kind of sample comprised experts, i.e., authors of scientific papers on stages of change in the Delphi study. As discussed in chapter 1, the Delphi study revealed a non-optimal response rate (38\%). Although the response did not seem to covary with researchers ${ }^{\prime}$ attitudes towards the TTM, the response might have biased the 
results. Since the Delphi results were mainly used to derive hypotheses and to construct the questionnaire for the longitudinal study, and not to test the theory, the non-optimal response rates may not be too problematic.

The second kind of sample comprised a random sample of members of an Internet panel. For the longitudinal study (chapters 2-5) a random sample of the panel from Flycatcher Internet Research BV (total panel size 8,165 individuals) was used, while for the experimental match-mismatch test (chapter 6) a random sample of the panel of Intomart GfK (total panel size 48,718 individuals) was used. Since only precontemplators and contemplators were invited to participate for this study, a larger sampling frame was needed. Further, using a different panel for the match-mismatch test reduced the risk that participants from the longitudinal cohort study were included in the sample again. The two panels produced similarly high response rates. The invitation to participate in a study yielded response rates of $62 \%$ and $65 \%$ for the Flycatcher and Intomart panels, respectively. Follow-up participation rates varied from $67 \%$ to $88 \%$ for the Flycatcher panel and from $82 \%$ to $90 \%$ for the Intomart panel. In the Flycatcher panel, non-completers were more often of foreign origin and were younger than completers. In the Intomart panel, no differences in demographic characteristics were found between completers and non-completers, though non-completers were more often in the precontemplation stage of change.

\section{Designs}

The studies that were conducted to gain more insight into the validity of the TTM employed strong designs, i.e., longitudinal and experimental. As was pointed out in the introduction of this thesis and earlier in this discussion section, research on the TTM mostly used cross-sectional designs. Although longitudinal designs do not allow for inferences on causation, they do allow conclusions about prediction and are thus stronger than cross-sectional designs (cf., Sutton, 1998).

\section{Measures}

The results described in the present thesis largely depend on the stage of change instrument. Correctly classifying individuals into only one of a limited set of stages of change is a precondition for successfully predicting stage transitions and matching interventions (Weinstein et al., 1998). For the research presented in this thesis, we used a one-item staging instrument with a five-option response format, congruent with most studies on TTM, for example with Nigg and coworkers (1999) and Laforge (1999). Also multiple-items instruments are available, such as the University of Rhode Island Change Assessment (URICA). However, research 
has shown that both instruments generally lead to a similar stage classification (Cook \& Perri, 2004). Single-items are advocated since they may reveal similar results with less burden to the respondent (Bowling, 2005; Patrician, 2004).

Furthermore, research comparing several methods revealed that a good stages of change instrument should include 1) a clear definition of the target behavior in terms of frequency, duration, and intensity, 2) measurement criteria that are understandable so that individuals can accurately stage themselves, and 3) a true/false response or five-choice response format for the algorithm (Reed, Velicer, Prochaska, Rossi, \& Marcus, 1997). The staging algorithm we used met these criteria.

However, there is debate about how to stage dietary behaviors, since misconception of consumption may be prevalent in such behaviors (Lechner et al., 1998; Ronda et al., 2001). It has been suggested that stages of change assessments for dietary behaviors should combine a traditional staging algorithm with more objective dietary assessments (e.g., food frequency questionnaires) (Kristal et al,, 1999; Ling \& Horwath, 2000; Ma et al., 2003). However, the TTM relies on self-report and argues that self-staging is important, since people will also have the cognitive mind-sets corresponding to their self-reported stage (Velicer et al., 1998). Since the present thesis attempted to test the TTM (and not an adaptation of the 'TTM), we did not include objective dietary assessments in the staging algorithm.

As discussed in chapter 3 , the processes of change measures need further validation, since a scale with only one item per process was used. The aggregated experiential and behavioral processes measures (with five internally consistent items each), operationalized as outlined by the TTM, were the point of departure in the analyses instead of the separate single item measures for separate processes.

\section{Strategy of analyses}

Predictors of forward stage transitions were analyzed with logistic regression. Due to the small number of respondents progressing through the stages, we used stage transition to a more advanced stage of change between two time points as the outcome, instead of stage transition to the next stage in the sequence. For example, precontemplators who make a forward stage transition include precontemplators who progress to contemplation, but also precontemplators who progress to preparation. The latter precontemplators actually make two stage transitions between two time points, i.e.; from precontemplation to contemplation, and from contemplation to preparation. This approach may have made it more complex to identify stage-specific predictors of stage transition. 
A true longitudinal analysis (e.g.r making use of all assessments, and not only two time points) would have been better. However, methods for longitudinal categorical data with varying time intervals are still lacking. In the past decade, a method, latent transition analysis (LTA), has been developed for analyzing longitudinal categorical data, and LTA has been used for longitudinal data on stages of change (Martin et al., 1996; Velicer, Martin, \& Collins, 1996). However, LTA still makes use of only two assessments in one analysis and continuous independent variables (such as pros and cons) cannot be included as yet. LTA was, therefore, not applied in the studies presented in this thesis.

\section{Internet research}

In all studies data were collected by means of electronic questionnaires. Additionally, in the match-mismatch test, web-based feedback was provided. Internal and external validity of collecting data via the Internet, and delivering feedback via the Internet will be discussed.

Online data collection can favor internal validity, since respondents enter the data directly into the database. Such an automatic data entry enhances the accuracy and speed of the data-entry process (Topp \& Pawloski, 2002). Further, electronic questionnaires provide possibilities to optimize questionnaire design, such as automatic routing of questions, or providing audio and visual stimuli to augment survey questions (Couper, Traugott, \& Lamais, 2001). Yet, psychological research on the Internet expanded only recently and research exploring and testing the validity of online data collection seems warranted (Kraut et al., 2004). It might be that potential respondents answer or read online questions in a different manner, although several studies have shown that web-based questionnaires and traditional print questionnaires may lead to similar responses and psychometric properties (Mangunkusomo et al., 2005; Riva, Teruzzi, \& Anolli, 2003).

Although external validity may be less important than internal validity in testing a theory, we strived for optimal external validity as well. Therefore, the longitudinal cohort study and the match-mismatch test were conducted in random samples from Internet panels of respondents. However, the results of the studies are generalizable at best only to Dutch adults with Internet access (Bush et al., 2004). In 2004, 74\% of the Dutch population had access to the Internet at home. A large part of the people who do not have access at home, have the opportunity to access the Internet elsewhere, for example at work, at school or at the public library. Older people and people with low incomes are less likely to have Internet access (SCP, 2004). In the cohort study, the respondents were indeed younger and higher educated than the Dutch population at large (see chapter 2), which might have reduced the external validity. From a different point of view, the Internet 
panels may have increased external validity, since on all assessments high response rates were established. Further, the Internet panels allowed for the inclusion of populations that are normally difficult to access (Nosek et al., 2002), such as precontemplators.

Another aspect of Internet research that needs to be discussed is the possibility to give immediate feedback (Topp \& Pawloski, 2002). In the matchmismatch test, for instance, the individualized feedback was provided immediately after the completion of the diagnostic tool (chapter 6). In contrast to printed individualized feedback, which normally takes days or even weeks between assessment and feedback delivery, web-based feedback minimizes the risk that beliefs, stage of change and behavior may already have changed between assessment and feedback (Brug et al., 2003). As argued in chapters 4 and 6, due to short-term stage instability, match-mismatch tests are particularly vulnerable to unintended matching or mismatching. Web-based feedback can be used to ascertain that an individual receives the correct intervention. However, it is not yet established whether behavior change activities using the Internet as the channel of delivery are effective and future research should provide a more definitive answer on this issue (De Nooijer, Oenema et al., 2005).

\section{Theoretical Reflections}

A sound theoretical framework should be based on empirical evidence, but should preferably also be practically applicable (Lewin, 1951; Rychlak, 1968). First, we discuss how the empirical results of the studies presented in this thesis should be interpreted and what they imply for the validity of the TTM. Next, the utility of the TTM is discussed. Finally, the validity and applicability of the TTM specifically for fruit intake is discussed.

\section{Empirical support for the Transtheoretical model}

It is important first to discuss what results would confirm or refute stage model requirements. Ogden (2003) argued that researchers who are testing social cognition models frequently conclude that the results provide support for a particular model, while the majority of these studies also revealed results not consistent with the predicted associations between constructs and the outcome variable. Data that are inconsistent with predictions from a particular model are often not used to reject a model. Instead, explanations are offered for the inconsistency, such as improper operationalization of constructs, sample 
characteristics or type of behavior (Ogden, 2003). To exemplify, we found that pros predict stage transitions, but cons did not predict early stage transitions, while the TTM postulates that both should be predictors. This can be explained in terms of operationalization of cons (items were not representative for the cons experienced) or type of behavior (fruit intake may not have many disadvantages) as can be read in chapter 2 , but these data can also be used to criticize or even reject the model since the results were not as hypothesized. Similar results, thus, may reveal different interpretations and conclusions. In a review of 87 studies on stages of change, Littell and Girvin (2002) showed that proponents of the TTM claim that empirical support for the TTM has been replicated repeatedly (e.g.* DiClemente \& Prochaska, 1998; Prochaska et al., 2003). However, studies that are cited to illustrate the evidence for TTM do often not actually fully support the TTM (Littell \& Girvin, 2002). Davidson also argues that many of the data taken as supportive of the TTM are more consistent with a continuous model (Davidson, 1998).

Thus, problems exist with how to derive conclusions from data on the TTM. This is exactly what makes it difficult to draw firm conclusions about the TTM's validity. With respect to two different philosophical perspectives on scientific theory, the TTM seems to fail. First, we could reason from a logical empiricist view, i.e., science should aim to confirm theory (Sarkar, 1996). The results described in this thesis did not confirm the existence of discrete, qualitatively different Transtheoretical stages of change. Second, as proposed in Popper's critical rationalism (Popper, 1959), researchers should seek to refute theory $y_{r}$ or at least be able to identify testable hypotheses that allow for the rejection of a scientific theory. The studies conducted to test stage model requirements did not allow for the formulation of such definite hypotheses that would (definitely) reject the TTM.

The results presented in this thesis did not fulfill stage model requirements for the three pre-action stages that are distinguished in the TTM (precontemplation, contemplation, and preparation); the stages were not qualitatively different, stage-matched interventions were not superior to stagemismatched interventions, and indications were found that the stages might as well represent pseudostages. As is argued in chapter 5, results that are not in line with the TTM might still be in line with a staging process of health behavior. Several alternative stage theories exist, such as the Health Action Process Approach (Schwarzer, 1992b), the Precaution Adoption Process Model (Weinstein, 1988), Bagozzi's Goal Theory (Bagozzi \& Edwards, 1998), and the Model of Action Phases (Heckhausen, 1991). While these models define different numbers of stages, they all share the idea that people move from no motivation, through motivation, to action (Armitage \& Conner, 2000). The problems that exist with the Transtheoretical pre-action stages may be overcome, by combining these pre-action stages into one motivational stage. Such a motivational stage is 
assumed to end with the formation of a positive intention. The next stage, the volitional stage, concerns the translation of this intention into behavior change: Finally, the initiated behavior changes should be maintained. Future research should test whether these models define genuine stages.

\section{The utility of the Transtheoretical model}

It has been argued that many theories that presume a continuous intention on behavior change, such as the Theory of Planned Behavior, do not provide clear guidelines for intervention development. These theories are, therefore, not particularly helpful in designing health behavior change interventions (Baranowski, Cullen, Nicklas, Thompson, \& Baranowski, 2003; Jeffery, 2004; Rothman, 2004). The TTM does attempt to specify the conditions under which specific constructs influence behavioral decisions. Further ${ }_{\nu}$ strategies and methods to change behaviors are suggested for each of the stages (Rothman, 2004), and the TTM thus may help to define different target group segments that require different intervention approaches. The great appeal the TTM has to practitioners and interventionists probably results from this presumed clear applicability. However, the effectiveness of Transtheoretical stage-based interventions has not been proven yet. Reviews on smoking cessation and lifestyle change in primary care settings did not find convincing evidence for the effectiveness of stage-based interventions (Riemsma et al., 2003; Van Sluijs et al., 2004). In a review of stagebased interventions related to exercise behavior, it was concluded that such interventions are not likely to induce longer-term behavior change. However, evidence for short-term changes in exercise behavior was found (Adams \& White, 2003, 2005). Stage-based interventions are almost all short-term (educational) interventions, and long-term behavior changes are not likely to result from such short-term strategies (Brug, Conner et al, 2005).

Although studies on stage-based interventions showed inconsistent effects, other approaches that were more or less inspired by stages of change have proven successful. For example, computer-tailored interventions have included individualized feedback partly driven by stages of change in addition to other variables, such as awareness, attitudes or self-efficacy (Brug et al., 1999). These tailored interventions are not stage-based in the sense that for each stage of cliange different psychosocial variables are addressed; tailored interventions provide individualized feedback and advice. Nevertheless, such individualized interventions that do take individuals' readiness to change into account have shown promising results, especially for dietary behavior change, i.e., fat, fruit and vegetable intake (Brug et al., 1999; Brug \& Van Assema, 2000; Kroeze et al., in press). Motivational interviewing is another behavior change approach, which takes an individual's readiness for change into account. Facilitating behavior 
change by helping individuals to explore and resolve their ambivalence about behavior change is the main focus of motivational interviewing (Miller \& Rollnick, 1991). Recent reviews showed positive effects of motivational interviewing for behavior change, e.g., in substance use and treatment adherence (Britt, Hudson, \& Balmpied, 2004; Rubak, Sandbaek, Lauritzen, \& Christensen, 2005).

A preliminary conclusion may be that although the validity of the TTM is disputed, some behavior change approaches that are inspired by thinking of behavior change in a stage-like manner seem promising.

\section{Validity and applicability of the Transtheoretical model for fruit intake}

In the studies described in chapters 2 to 6 the TTM was applied to fruit intake. When stage model requirements are tested, the model's predictions should indeed be preferably tested for a concrete behavior (Weinstein et al., 1998). Testing one concrete behavior in several studies allows for the elaboration on the results for each of the studies, but it limits the generalization of the results to other types of behaviors. The existence of separate pre-action Transtheoretical stages of change could not be confirmed for fruit intake in the studies presented in this thesis. The TTM was originally developed for addictive behaviors (e.g., smoking cessation), for which often abstinence or limitation is recommended. As is argued in chapters 3 and 6 , fruit intake is a continuous behavior and increasing intake instead of limiting or cessation is recommended, and the validity of the TTM has not previously been examined in detail for such behaviors.

Different behavior theories operate on different levels of generality. A key distinction can be made between content-free models and content-specific models (Ajzen, 1998). Content-free models should be applicable to virtually any behavior: the Theory of Planned Behavior and Social Cognitive Theory are examples of content-free models. Such models stipulate a small set of constructs that motivate behavior across behavioral domains. The importance of each of the constructs may vary from behavior to behavior. Further, the models' boundaries should be acknowledged. For example, the Theory of Planned Behavior (as well as the TTM) can only be applied to intentionally controlled behaviors (i.e., behaviors for which individuals can consider the implications before they decide to act). It may not be of much use to apply these models to behaviors with restricted intentional control, for example due to environmental influences (e.g., habits or legislation; cf., Ajzen \& Fishbein, 1980).

Content-specific models deal with specific issues, such as health and illness, or a specific behavior or health outcome. These models outline specific factors that must be considered to understand particular health-related behaviors (Ajzen, 1998). An example of a content-specific model is the ANGELO framework 
(analysis grid for environments linked to obesity), which is a conceptual model for understanding the obesogenicity of enwironments, and a practical tool for prioritizing environmental elements for research and intervention on obesity (Swinburn, Egger, \& Raza, 1999).

The TTM is treated as a content-free model, with applications in various fields of behavior, such as health behavior and consumer behavior (Prochaska et al. 2003). Our results showed that the TTM may not be valid when applied to frutt intake. One might argue that fruit intake is a behavior that falls outside the TTM's boundaries of intentional control. However, different studies have shown that intentions do predict fruit intake (e.g., chapters 3 and 5, Bogers, Brug, Van Assema, \& Dagnelie, 2004; Brug, De Vet, De Nooijer, \& Verplanken, in press). It could also be that the TTM is not a content-free model, in that its use is restricted to addictive behaviors for which cessation is often recommended. The TTM would require the construction of a different model for different subclasses of behavior (Ajzen, 1998), such as continuous behaviors like increasing fruitt intake. Therefore, it seems advisable to test stage model requirements for behaviors, other than addictive behaviors, in order to successfully apply the TTM to such behaviors.

\section{Implications}

This thesis described the results of different tests of the TTM as applied to fruit intake. The results have several implications for future research as well as for health education practice. The implications for future research focus on the further testing of the TTM as well as other stage models. The implications for health education practice focus on the application of the TTM in future health behavior change interventions.

\section{Implications for research}

In the discussion of the results, methodological issues and theoretical reflections, several recommendations were already presented for future research. The implications for research described in this section broadly suggest further stage model testing. As is discussed in the theoretical reflections, although the TTM may not be valid enough, behavior change might still be a staged process. Two directions in future stage model research could be fruitful. First, the studies presented in this thesis almost all focus on precontemplation and contemplation. Future TTM studies should test stage model requirements for the preparation, 
action and maintenance stages. Second, we argued that other stage models might capture the behavior change process better than the TTM does. In particular, the three-phase models with a motivational, volitional, and maintenance phase deserve attention, and stage model requirements for such stage models should be tested. All following suggestions for future research, thus, refer to further TTM testing (e.g. later stages of change) as well as to testing other stage models (e.g., three-phase models).

One avenue for future research relates to the stage model requirement of stage-specificity. In this thesis, stage-specific predictors were examined in a longitudinal cohort study. Future tests of stage transition determinants should use experimental designs rather than prospective designs to gain insight into the explanatory nature of the determinants. With experimental designs more insight can be obtained into the nature of stage-specificity. Earlier in this discussion section we presented four perspectives on the nature of stage-specificity that should be acknowledged in future studies. We should aim to answer four important questions by means of such experimental research. First, do predictors of stage transitions also explain these transitions? In other words, does an experimental manipulation of a predictor of stage transition (e.g., self-efficacy) lead to stage transition? Second, are stage transition determinants stage-specific? Does a manipulation of such a predictor lead to a single stage transition, or does the manipulation produce more than one stage transition? Third, what is the effect on stage transition and behavior change of targeting multiple determinants simultaneously compared to targeting single determinants, and compared to targeting multiple determinants in a sequential fashion (i.e., targeting relevant determinants one after the other)? Fourth, does the effect on stage transition depend on the type of manipulation? More specifically, do different manipulations of, for example, self-efficacy (such as modeling or mastery experiences) have stage-specific effects on stage transition? Further, these experimental tests can be used to gain more insight into the proposed causal chain, in which cognitive changes are supposed to evoke intentional changes, which in turn should lead to behavioral changes.

Another avenue for future research on stage models relates to comparing, combining and contrasting theories. Prochaska and DiClemente (1998, p. 43) stated that "models are not meant to be assessed by absolute criteria. For us a key question is how well the TTM performs relative to any other leading theories". It has repeatedly been argued that many of the health behavior theories share commonalities, though these similarities are often neglected (Weinstein, 1993). As Ajzen phrased it, "little is gained by needlessly multiplying predictors, using different labels for basically the same construct, or otherwise duplicating theoretical efforts"r (Ajzen, 1998, p. 736). The major purpose of theories, such as the TTM, is to understand health behavior, and to help us design effective behavior change interventions. Comparing and contrasting theories can probably 
teach us more about health behavior than the study of any theory in isolation (Nigg et al., 2002). Practitioners and applied scientists use theory from a problemdriven perspective (aimed at tackling a specific [health] problem) and they will use theory to fully understand the causes; consequences and solutions of a problem. Thus, for problem-solving purposes a multiple-theory and integrative perspective would be more fruitful than a single theory with only a limited set of factors relevant to the problem under investigation (Brug, Oenema et al., 2005). In the future, more efforts should be taken to compare, combine and contrast theories. For example, stage models can be compared to each other, but also to other leading theories in the field. Also, within stage models, various other specific theories can be applied.

\section{Implications for practice}

Now that it is concluded that the TTM may not be a valid stage model, at least not when applied to fruit intake, one might cast doubts about using the TTM in practice. However, some stages of change and some other TTM components have proved to be useful in describing and predicting various health behaviors, including fruit intake. We may, therefore, not completely stop thinking about behavior change in a stage-like manner. As is argued in this discussion section, TTM-inspired interventions, which use methods such as motivational interviewing or computer-tailoring, might yield promising results. However, rigorous TTM-based interventions, with separate stage-appropriate interventions and delivery in a sequential order, might not necessarily produce better results than TTM-inspired interventions.

Probably, the most important lesson learned from the TTM is that a large proportion of at-risk populations may not be ready for behavior change and will not be helped by traditional action-oriented programs (Prochaska \& Velicer, 1997a). Practitioners should acknowledge these individuals, whether from the perspective of the TTM or from that of another stage model, by planning to recruit these individuals to participate in interventions, and by assuring that those individuals receive health-promoting interventions correspondung to their readiness of change.

Furthermore, we recommend that if the TTM is applied to a behavior other than addictive behavior, the relevant beliefs and predictors of stage transition for the target behavior should be identified prior to developing interventions. The determinants of stage progression may be different from those proposed in the TTM. Our Delphi study indicated that experts in the field already do acknowledge a much broader spectrum of potential stage transition determinants than proposed within the TTM boundaries. Next, when an intervention tailored to individuals" readiness is planned to be delivered, the time 
lag between assessment of readiness and intervention delivery should be as short as possible, because stage transition may occur within short time intervals. This also means that caution is warranted when using stages of change as an indicator of intervention impact.

\section{Concluding remark}

The TTM of behavior change is a widely used model in understanding health behaviors and in designing and evaluating behavior change interventions. The TTM might have pragmatic value in describing health behavior change. However, the presented studies showed that the TTM might not be a valid stage model when applied to fruit intake. The main outcomes are outlined below:

- The studies showed that cross-sectional correlates of being in a particular stage differ from longitudinal predictors of stage transition. Researchers should aim to study stage transitions instead of crosssectional differences between stages of change.

- The Transtheoretical pre-action stages of change are not qualitatively different. Our studies showed that predictors of stage transitions were not stage-specific.

- Stage transitions in a natural setting mostly involve transitions to an adjacent stage of change.

- The Transtheoretical pre-action stages may reflect a categorization of a continuous intention measure (i.e., pseudostages) instead of discrete stages.

- The randomized trial did not provide evidence that individualized stage-matched interventions are superior to individualized stagemismatched interventions.

- It is recommended that future research should aim at gaining more insight into the validity and applicability of other stage models. 


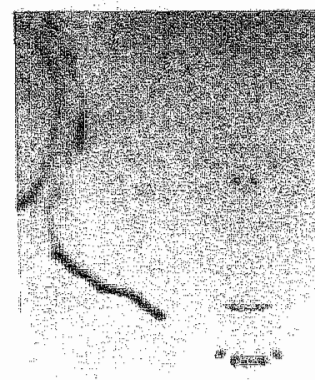

\section{References}

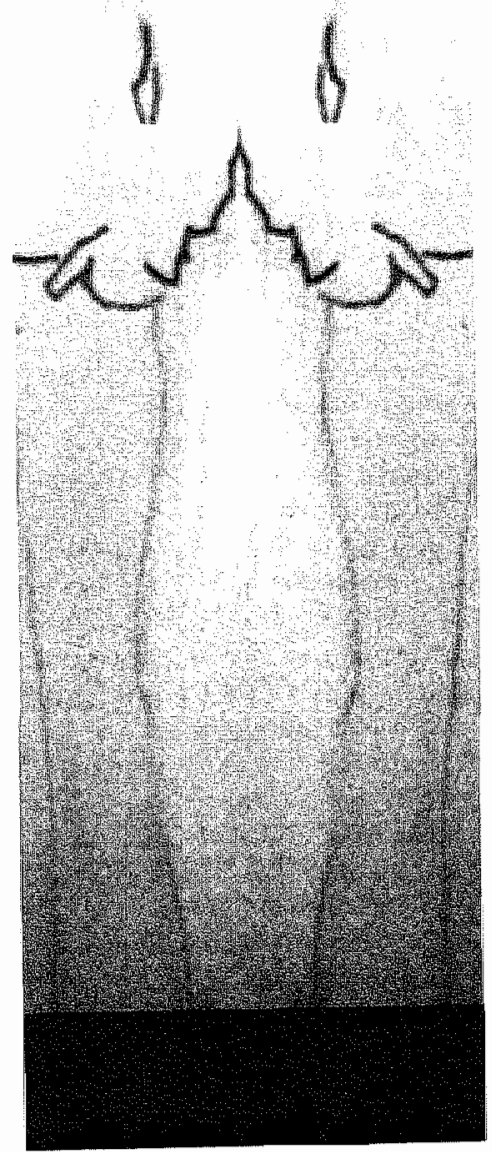


$x^{2}+3+2 x^{2}+x^{2}$

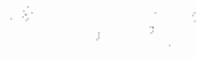




\section{References}

Abraham, C. \& Sheeran, P. (2003). Acting on intentions: the role of anticipated regret. British joufnal of Social Psychology, $42,495-511$.

Adams, I. \& White M. (2003). Are activity promotion interventions based on the transtheoretical model effective? A critical review. British Journal of Sports Meidicine, 37, 106-114.

Adams, I., \& White, M. (2005). Why don"t stage-based activity promotion interventions work? Heallh Education Research, 20, 237-243.

Ajzen, I. (1991). The theory of planned behavior. Organizational Behawior and Human Decision processes, $50,179-211$.

Ajzen, 1. (1998). Models of human social behavior and their application to health psychology. Psychology and Health, 13, 735-739.

Ajzen, I., \& Fishbein, M. (1980). Understanding Attitudes and Predicting Social Behavior. Englewood Cliffs, NJ: Prentice-Hall, Inc.

Armitage, C. I., \& Arden, M. A. (2002). Exploring discontinuity patterns in the transtheoretical model: an application of the theory of planned behaviour. British Journal of Health Psychology, 7,89 . 103.

Armitage, C. I. \& Conner, M. (2000). Social cognition models and health behaviour: a structured review. Psychology and Health, 15, 173-189.

Armitage, C. J., Povey, R. \& Arden, M. A. (2003). Evidence for discontinuity patterns across the stages of change: a role for attitudinal ambivalence. Psychology and Health, 18, 373-386.

Armitage, C. J., Sheeran, P., Conner, M, \& Arden, M. A. (2004). Stages of change or changes of stage? Predicting transitions in transtheoretical model stages in relation to healthy food choice. Joumal of Consulting and Clinical Psychology, 72, 491-499.

Aveyard, P. Griffin, C., Lawrence, T., \& Cheng, K. K. (2003). A controlled trial of an expert system and self-help manual intervention based on the stages of change versus standard self-hellp materials in smoking cessation. Addiction, 98, 345-354.

Bagozzi, R. P. \& Edwards, E. A. (1998). Goal setting and goal pursuit in the regulation of body weight. Psychology and Health, 13, 593-621.

Bandura, A. (1977). Social Learning Theory. New Jersey: Prentice Hall.

Bandura, A. (1982). Self-efficacy mechanism in human agency. American Psychologist, 37, 122-147.

Bandura, A. (1986). Social foundations of thought and action. Englewood Cliffs: Prentice-Hall.

Bandura, A. (1997). The anatomy of stages of change. American Journal of Healih Promotion, 12, 8-10.

Bandura, A. (1998). Health promotion from the perspective of social cognitive theory. Psychology and Heralth, 13, 623-649.

Baranowski, T., Cullen, K. W., Nicklas, T., Thompson, D., Baranowski, J. (2003). Are current health behavioral change models helpful in guiding prevention of weight gain efforts. Obesity Research, $11,239-435$.

Bem, D. J. (1967). Self-perception: an alternative interpretation of cognitive dissonance. Psychological Review, 74, 183-200.

Blissmer, B., \& McAuley, E. (2002). Testing the requirements of stages of physical activity among adults: the comparative effectiveness of stage-matched, mismatched, standard care and control interventions. Annals of Behartoral Medicine, 24, 1.81-189.

Boer, H., \& Seydel, E. R. (1996). Protection motivation theory. In M. Conner \& P. Niorman (Eds.), predicting health behwriour: research and practice with social cognition models (pp. 95-120). Buckingham, UK: Open University Press.

Bogers, R. P., Brug, II., Van Assema, P.. \& Dagnelie, P. C. (2004). Explaining fruit and vegetable consumption: the theory of planned behaviour and misconception of personal intake levels. Apperite, 42, 157-166.

Bogers, R. P., Van Assema, P., Kester, A. D. M., Westerterp, K. R., \& Dagnelie, P. C. (2004), Reproducibility, validity and responsiveness to change of a short questionnaire for measuring intake of fruits and vegetables. Anterican Journal of Epidemiology, 159, $900-909$.

Bowen, D. J., Meischke, H., \& Tomoyasu, N. (1994). Preliminary evaluation of the processes of changing to a low-fat diet. Health Education Resenrch, 9, 85-94. 
Bowerman, B. L. \& O'Connell, R. T. (1990). Linear statistical models: an applied approach (2nd edition). Belmont, CA: Duxbury Press.

Bowling, A (2005). Just one question: if one question works, why ask several? Joumal of Epiderniology and Community Health, 59, 342-345.

Britt, E., Hudson, S. M., \& Balmpied, N. M. (2004). Motivational interviewing in heallh settings: a review. Patient Education and Counseling, 53, 147-155.

Brug. I. Campbell, M. \& Van Assema, P. (1999). The application of computer-generated personalized nutrition education: a review of the literature. Patient Education and Counseling, 36, 145-156.

Brug, J. Conner, M. Harre, N., Kremers, S., McKellar, S., \& Whitelaw, S. (2005). The transtheoretical model and stages of change: a critique. Observations by five commentators on the paper by Adams, J. and Whitte M. (2004) why don't stage-based activity promotion interventions work. Health Education Research, 20, 244-258.

Brug, 1., De Vet, E., De Nooijer, J., \& Verplanken, B. (in press). Predicting fruit consumption: cognitions, intention and habits. Journal of Nutrition Education and Behtavior

Brug, J., Glanz, K, \& Kolk, G. (1997). The relationship between self-efficacy, attitudes, intake compared to others, consumption; and stages of change related to fruit and vegetables. American Joutual of Health Promotion, $12,25-30$.

Brug, I., Oenema, A. \& Campbell, M. (2003). Past, present and future of computer-tailored nutrition education. American Jownal of Clinical Nutrition, 77, 10285-1034S.

Brugg J., Oenema, A., \& Ferreira, I. (2005). Theory, evidence and Intervention Mapping to improve behavior nutrition and physical activity interventions. International Journal of Behavioral Nutrition and Physical Activity; 2 ; doi: 10.1186/1479-5868-1182-1182.

Brugg J., \& Van Assema, P. (2000). Differences in use and impact of computer-tailored dietary feedback according to stage of change and education. Appetite, 34, 285-293.

Burke, V., Richards, J., Milligan, R. A. K., Beilin, L. J., Dunbar, D., \& Gracey, M. P. (2000). Stages of change for health-related behaviours in 18 year-old Australians. Psychology and Health, 14, $1061-1075$.

Bush, N. E., Bowen, D. I., Wooldridge, J., Ludwig, A., Meischke, H., \& Robbins, R. (2004). What do we mean by Internet access? A framework for health researchers. Preventing Chronic Diseatie, Retrieved April 22th, 2005, from http:/www.cdc.gvo/pod/issues/2004/oct/04_0019.htm

Campbell, M. K., Reymolds, K. D. Havas, S., Curry, S., Bishop, D., Nicklas, T, et al. (1999). Stages of change for increasing truit and vegetable consumption among adilts and young adults participating in the national 5-a-day for better health community studies. Health Education \& Behavior, 26, 513-534.

Campbell, M. K., Symons, M., Demark-Wahnefried, W., Polhamus, B., Bernhardt, I. M., McClelland, J. W, et al. (1998). Stages of Change and Psychosocial Correlates of Fruit and Vegetable Consumption Among Rural African-American Cintrch Members. American Journal of Health Prowotion, 12, 1.85-191.

Clark, P. G., Nigg, C. R., Greene, G., Riebe, D., \& Saunders, S. D. (2002). The Study of Exercise and Nutrition in Older Rhode Islanders (SENIOR): translating theory into research. Health Education Research, 17, $552-561$.

Clark-Carter, D. (2003) Effect size: the missing piece in the jigsaw. The Psychologist, 16, 636-638.

Cohen, J. (1988). Statistical powet analysis for the behavioral sciences (2nd ed.). Hillsdale, NJ: Lawrence Erlbatum.

Cook, C. L. Perri, M. (2004). Single-item versus multiple-item measures of stage of change in compliance with prescribed medications. Psychological Reports, 94, 115-124.

Couper, M. P. (2000). Web surveys. A review of issues and approaches. Public Opinion Quarterly, 64, $464-494$.

Couper, M. P., Traugott, M. W., \& Lamais, M. J. (2001). Web survey design and administration. Public Opinion Quarterly, 65, 230-253.

Coumeya, K. S. Plotnikoff, R. C., Hotz, S. B. \& Birkett, N. J. (2001). Predicting exercise stage transitions over two consecutive 6-month periods: A test of the theory of planned behaviour in a population-based sample. British Jowmal of Heal th Psychology, $6,135-150$.

Davidson, R. (1998). The transtheoretical model: a critical overview. In W. R. Miller \& N. Heather (Eds.), Treating addictive behaviors (2nd ed., pp. 25-38). New York: Plemum. 
De Bourdeaudhuij, $\mathrm{l}_{\text {, \& }}$ Brug, J. (2000). Tailoring dietary feedback to reduce fat intake an intervention at the family level. Heal h education research, $15,449-462$.

De Meyrick, J. (2003). The Delphi method and health research. Health Edwcation, 103, 7-16.

De Noodjer, J, Oenema, A, KJoek, G., Brug, H., De Vries, H., De Vries, N. (2005). Berorderting van

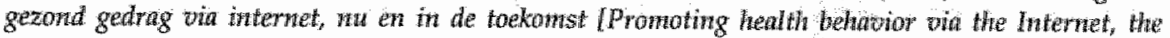
present and the future]. Rotterdam: Optimat Grafische Communicatie.

De Nooijer, J., Van Assema, $\mathbb{P}_{.}$De Vet, E., Brug, J. (2005). How stable are stages of change for nutrition behaviors? Health Promotion International, 20, 27-32.

De Vet, E., Brug, I., De Nooijer, J.r Dijkstra, A., \& De Vries, N. K. (2005). Deterninants of forward stage transitions: a Delphi study. Health Education Research, 20, 195-205.

De Vet, E., De Nooijer, J., De Vries, N. K., \& Brug, J. (2005a). Determinants of forward stage transition from precontemplation and contemplation for fruit consumption. Americmi Jotrnal of Hetalth Pronsotion, 19, 278-285.

De Vet, E., De Nooijer, J., De Vries, N. K., \& Brug, J. (2005b). Stages of change in fruil intake: a longitudinal examination of stability, stage transitions and transition profiles. Psychology and Health, 20, 415-428.

De Vet, E., De Nooijer, J., De Vries, N. K., \& Brug J. (submitted). Do the transtheoretical processes of change predict transitions in stages of change for fruit intake?

De Vries, $H_{n}$, \& Backbier, E. (1994). Self-efficacy as an important determinant of quitting among pregnant women who smoke: the Ø-pattern. Preventive Medicine, 23, 167-174.

De Vries, H., \& Mudde, A. N. (1998). Predicting stage transitions for sinoking cessation applying the attitude-social influence-efficacy model. Psychology and Health, 13, 369-385.

De Vries, H., Mudde, A. N., Dijkstra, A., \& Willemsen, M. C. (1998). Differentiall beliefs, perceived social influences, and self-efficacy expectations among smokers in various motivational phases. Preventive Medicine, 27, 681-689.

De Vries, H., Mudde, A. N., Leijs, L., Charlton, A., Vartiainen, E., Buijs, G., ef al. (2003). The European smoking prevention framework approach (ESFA): an example of integral prevention. Heallh Education Research, 18, 611-626.

DiClemente, C. C., \& Prochaska, J. O. (1998). Toward a comprehensive transtheoretical model of change. Stages of change and addictive behaviors. In W. R. Miller \& N. Heather (Eds.), Treating addictioe behaviors (2nd ed., pp. 3-24). New York: Plenum.

Dijkstra, A., Bakker, M., \& de Vries, H. (1997). Subtypes within a sample of precontemplating smokers: A preliminary extension of the stages of change. Healh Education Research, 22, 327-337.

Dijkstra, A., \& De Vries, H. (2001). Do self-help interventions in health education lead to cognitive changes, and do cognitive changes lead to behavioural change. British Joutnal of Health Psychology, 6, 121-134.

Dijkstra, A., De Vries, H., Roijackers, J. \& Van Breukelen, G. (1998). Tailored interventions to communicate stage-matched information to smokers in different motivational stages. Joumal of Consulting and Clinical Psychology, 66, 549-557.

Dijkstra, A., Tromp, D. \& Conijn; B. (2003). Stage-specific psychological determinants of stage transition. British Journal of Health Psychology, 8, 423-437.

Dutch Cancer Society (2004). De rol wan voeding bij het ontstaan vasn kanker the role of diet in the development of cancerl. Den Haag: KWF Kankerbestrijding.

Dutch Nutrition Centre (1998). Zo eet Nederland IThis is how the Dutch eat. Den Haag: Voedingscentrum.

Eagly, A. H. \& Chaiken, S. (1998). Attitude Structure and Function. In D. Gilbert, S. T. Fiske \& G. Lindzey (Eds), The Handbook of Social Psychology (pp. 269-322). New York: McGraw-Hill.

Etter, J. F., \& Perneger, T. V. (1999). Associations between the stages of change and the pros and cons of smoking in a longitudinal study of Swiss smokers. Adtictive Behaviors, 24, 419-424.

Fava, I. L., Vellicer, W. F., \& Prochaska, J. O. (1995). Applying the ranstheoretical model to a representative sample of smokers. Addictive Behaviors, 20, 189-203.

Festinger, L. (1957). A theory of cognitive dissonance. Stanford, CA: Stanford University Press.

Fleiss, J. L. (1981). The measurement of interrater agreement. In J. L. Fleiss (Ed.), Statistical Methods for Rates and Proportions (2nd ed., pp. 212-236). New York: John Wiley \& Sons. 
Fontes de Oliveira, M. D. C. Anderson, $J_{n}$ Auld, G. $_{*}$ \& Kendall, P. (2005). Validation of a tool to measure processes of change for fruit and vegetable consumption among male college students. Jourwal of Nutrition Education and Behazior, 37, 2-11.

Gielen, A. C., Fogarty, L. A., Armstrong, K., Green, B. M., Cabral, R., Milstein, B., et al. (2001). Promoting condom use with main partners: a behavioral intervention trial for women. ANDS and Behavior, $5,193-204$.

Gollwitzer, P. M. (1999). Implementation intentions: Strong effects of simple plans. Americant Psychologist $, 54,493-503$.

Green, L. W., Kreuter M. W. (1999). Health Promotion Planning: an educational and environmental approuch (3rd ed.). Mountain View, CA: Mayfield.

Greene, G. W., Fey-Yensan, N., Padula, C., Rossi, S., Rossi, J. S., \& Clark, P. G. (2004). Differences in psychosocial variables by stage of change for fruits and vegetables in older adults. Journal of the American Diatetic Association, 104, 1236-1243.

Greene, G. W. \& Rossi, S. R. (1998). Stages of change for reducing dietary fat intake over 18 months: Journal of the American Dietetic Association, 98, 529-534.

Greene, G. W., Rossi, S. R. Rossi, J. S., Velicer, W. F., Fava, J. L., \& Prochaska, I. O. (1999). Dietary applications of the stages of change model. Joumal of the American Ditetic Associntion, 99, 673-678.

Hardeman, W. Johnston, M., Johnston, D. W., Bonetti, D., Wareham, N. J., \& Kinmonth, L. (2002). Application of the theory of planned behaviour in behaviour change interventions: a systematic review. Psychology wind Health, 17, 123-158.

Heckhausen, H. (1991). Motivation and action. Berlin: Springer.

Herrick, A. B., Stone; W. J., \& Mettler, M. M. (1997). Stages of change, decisional balance, and selfefficacy across four health behaviors in a worksite environment. American foumal of Health Promotion, 12, 49-56.

Herzog, T. A., Abrams, D. B., Emmons, K. M., Linnan, L. A., \& Shadel, W. G. (1999). Do processes of change predict smoking stage movements? A prospective analysis of the transtheoretical model. Hewlth Psychology, 18, 369-375.

Horacek, T. M., White, A., Betts, N. M., Hoerr, S., Georgiou, C., Nitzke, S., et al. (2002). Self-efficacy, perceived benefits, and weight satisfaction discriminate among stages of change for fruit and vegetable intakes for young men and women. Journal of the American Dietetic. Association, 102, 1466-1470.

Janis, L. L., \& Mann, L. (1977). Decision Making: A psychological analysis of conflict, choice and commitment. New York: Free Press.

Jeffery, R. W. (2004). How can health behavior theory be made more useful for intervention research? International Joumal of Behatioual Nutrition and Physical Activity, 1, doi: 10.1186/1479-5868$1181-1110$.

Jones, I. Hunter, D. (1995). Consensus methods tor medical and health services research. British Medical Joumal, 311, 376-380.

Jordan, P. J., \& Nigg, C. R. (2002). Applying the transtheoretical model: tailoring interventions to stages of change. In P. M. Burbank \& D. Riebe (Eds.), Promoting exercise and behavior change in older adults: interventions with the transtheoretical model (pp. 181-207). New York: Springer Publishing co.

Key, T. I., Allen, N. E., Spencer, E. A., \& Travis, R. C. (2002). The effect of diet on risk of cancer. Lancet, $360,861-868$

Key, T. Io, Schatzkin, A, Willet, W. C.y Allen, N. E., Spencer, E. A., \& Travis, R. C. (2004). Diet, nutrition and the prevention of cancer. Public Heallh Nutrition, 7, 187-200.

Kloek, G. C., Van Lenthe, F. J., Van Nierop, P. W. M., Mackenbach, J. P. (2004). Stages of change for fruit and vegetable consumption in deprived neighborhoods. Hedlh Education \& Behavior. 31, 223-241.

Kok, G., Schaalma, H., De Vries, H., Parcel, G., \& Paulussen, T. (1996). Social psychology and health education. Europeran Review of Social Psychology, 7, 241-282.

Kok, G. Schaalma, H., Ruiter, R. A. C., Van Empelen, P., \& Brug, J. (2004). Intervention Mapping: A protocol for applying health psychology theory to prevention programmes. Journal of Health Psychology, 9,85-98. 
$K_{\text {Kok }}$ G.; Van den Borne, B, \& Dolan Mullen, P. (1997). Effectiveness of health-education and health promotion: meta-analyses of effect studies and determinants of effectiveness. Patient Education and Counseling, 30, 19-27.

Kraemer, H. C. (2004). Reconsidering the odds ratio as a measure of $2 \times 2$ association in a population. Statistics in Medicine, 23, 257-270.

Kraemer, H. C., Willson, T. Fairburn, C. G., \& Agras; W. S. (2002). Medtiators and moderators of treatment effects in randomized clinical trials. Archives of General Psychiatry, 59, 877-883.

Kraft, P., Sutton, S. R., \& MicCreath-Reynolds, H. (1999). The transtheoretical model of behaviour change: are the stages qualitatively different? Psychology and Heal th, 14,433-450.

Kraut, R., Olson, J.s Banaji, M., Bruckman, A., Cohen, J.; \& Couper, M. (2004). Psychological research online. Report of board of scientific affairs' advisory group on the conduct of research on the Internet. Anterican Psychologist, 59, 105-117.

Kremers, S. P. J. Mudde, A. N., \& De Vries, H. (2001). Subtypes within the precontemplation stage of adolescent smoking acquisition. Addictive Behartiors, 26, 237-251.

Kremers, S. P. J., Mudde, A. N., \& De Vries, H. (2004). Model of unplanned smoking initiation of adolescents: an integrated stage model of smoking behavior. Ppeventive Medicine, 38, 642-650.

Kristal, A. R., Glanz, K., Curry, S. I., \& Patterson, R. E. (1999). How can stages of change be best used in dietary interventions? Journal of the American Dietetic .Association, 99, 679-684.

Kristal, A. R., Hedderson, M. M., Patterson, R. E. \& Neuhauser, M. L. (2001). Predictors of selfinitiated, healthful dietary change. Joumal of the American Dieteric Association, 101, 762-766.

Kroeze, W., Werkman, A., \& Brug, J. (in press). A systematic review of randomized studies on the effects of computer-tailored health education for diet and physical activity change. Anmals of Behavioral Medicine.

Kruglanski, A. W. (2001). That "vision thing": the state of theory in social and personality at the edge of the new millenium. Journal of Personality and Social Psychology, 80.871-875.

Laforge, R. Gux, Greene, G. W., \& Prochaska, J. O. (1994). Psychosocial factors influencing low fruit and vegetable consumption. Journal of Behavioral Medicine, 17, 361-374.

Laforge, R. G., Velicer, W. F., Richmond, R. L., \& Owen, N. (1999). Stage distributions for five health behaviors in the United States and Australia. Preventive Medicine, 28, 61-74.

Lam, T. H., Chan, B., Ho, S. Y., \& Chan, W. M. (2004). Stage of change for general health promotion action and health-related lifestyle practices in Chinese adults. Preventive Medicine, 38, 302308.

Lamb, R., \& Joshi, M. S. (2004). Active but not consistent: dietary behaviour and the stages of change model. Psychology and Heal th, 19, 543-559.

Lanza, E., Schatzkin, A., Daston, C, Corle, D. K., Freedman, L. S., Ballard-Barbasch, R., et al. (2001). Implementation of a 4-year, high-fiber, high-fruit and -vegetable, low-fat dietary intervention: results of dietary chariges in the Polyp Prevention Trial. American Jowrnal of Clinical Nutrition, 74, 387-401.

Lechner, L., Brug J., \& De Vries, H. (1997). Misconception of fruit and vegetable consumption: interpretation and consequences. Jow of Wultition Education and Behatior, 29, 313-320.

Lechner, L., Brug, J., de Vries, H., van Assema, P. Mudde, A. (1998). Stages of change for fruit, vegetable and fat intake: consequences of misconception. Health Education Reseatch, 13, 1-11.

Lewin, K. (1951). Field theory in social science. New York: Harper and Row.

Ling, A. M.C., \& Horwath, C. (2000). Defining and measuring stages of change for dietary behaviors: readiness to meet fruit, vegetable, and grain guidelines among Chinese Singaporeans. Jountal of the American Dietetic Association, 108, 898-904.

Ling, A. M. C. \& Horwath, C. (1999). Self-efficacy and consumption of fruit and vegetables: Validation of a summated scale. American Jowrnal of Heal th Promotion, 13, 290-298.

Ling, A. M. C., \& Horwath, C. (2001). Perceived benefits and barriers of increased fruit and vegetable consumption: validation of a decisional balance scale. Journal of Nutrition Education and Beharvior, 33, 257-265.

Linstone, H. A. \& Turoff, M. (1975). The Delphi Method: Techniques and Applications. Massachusetts: Addison-Wesley Publishing Company.

Littell, J. H., Girvin, H. (2002). Stages of change: a critique. Behavior Modification, 26, 223-273. 
Ma, I, Betts, N. M. \& Horacek, T. (2001). Measuring stage of change for assessing readiness to increase fruit and vegetable intake among 18- to 24-year-olds. Americar Joumal of Health prownotion, 16, 88-97.

Ma, I, Betts, W. M., Horacek, T., Georgion, C, \& White, A. (2003). Assessing stages of change for fruit and wegetable intake in young adults: a combination of traditional staging algorithms and food-frequency questionnaires. Health Education Reseapch, 18, 224-236.

Ma, I. Betts, N. M. Horacek, T., Georgiou, C., White, A, \&itzke, S. (2002). The importance of decisional balance and self-efficacy in relation to stages of change for fruit and vegetable intakes by young adults. American Jourmal of Heal h promotion, 16, 157-166.

Maclure, M. (1998). Mechanistic versus empirical explanations and evidence-based medicine. Acta Oncologica, $37,11-12$.

Mangunkusomo, R. T, Moormani, P. W, Van den Berg-de Ruiter, A. E, Van der Lei, J., De Koning, H. I., Raat, $\mathrm{H}$. (2005). Internet-administered adolescent health questiontinaires compared with a paper version in a randomized study. Journal of Adolescent Health, 36, 70.e1-70.e6.

Marlatt, G. A., Gordon, J. R. (1985). Relapse prevention: maintenance strategies in the treatment of addicline behaviors. New York. The Guilford Press.

Marsh, H. W. (1994). Sport motivation orientations: beware of jingle-jangle fallacies. Journal of Sport \& Extrise Psychology, 16, 365-380.

Marshal1, S. I. \& Biddle, S. J. H. (2001). The transtheoretical model of behavior change: a meta-analysis of applications to physical activity and exercise. Annals of Behavioral Medicine, 23, 229-246.

Martin, R. A., Velicer, W. F. \& Fava, J. L. (1996). Latent transition analysis to the stages of change for smoking cessation. Addictive Behiaviors, 21, 67-80.

MoKema, H. P. (1994). The Delphi technique: a worthwile research approach for nursing? Journal of Aduarced Nursing 19, 1221-1225.

McKenna, $\mathrm{T}_{0}$ \& $\mathrm{F}_{\mathrm{r}}$ rancis, $\mathrm{C}$. (2003). Exercise contemplators: unravelling the processes of change. Health Education, 103, 41-53.

Michie, S., \& Abraham, C. (2004). Interventions to change health behawiours: evidence-based or evidence-inspired? Psychology and Healh 19, 29-49.

Miller, W. R., \& Rollnick, S. (1991). Motivational interwiewing: preparing people to change addictive behawior. New York: Guilford Press.

Milne, S., Orbell, S., \& Sheeran, P. (2002). Combining motivational and wolitional interventions to promote exercise participation: Protection motivation theory and implementation intentions. British Joumal of Health Psychology, 7, 163-184.

Naylor, P. J., Simmonds, G., Riddoch, C., Velleman, G. \& Turton, P. (1999). Comparison of stagematched and unmatched interwentions to promote exercise behaviour in the primary care setting. Health Educration Reserarch, 14,653-666.

Ni Mhurchu, C, Margetts; B. M. \& Speller, V. M. (1997). Applyüng the stages-of-change model to dietary change. Nutrition Reviezos, 55, 10-16.

Nigg, C. R. (2001). Explaining adolescent exercise behavior change: a longitudinal application of the transtheoretical model. Annals of Behrowional Medicine, 23, 11-20.

Nigg, C. Ra, Alllegrante, J. P., \& Ory, M. (2002). Theory-comparison and multiple-behavior research: common themes advancing health behavior research. Health Education Research, 17, 670-679.

Nigg, C. R., Burbank, $\mathbb{P}$. M., Padula, C, Dufresne, R., Rossi, J. S, Velicer, W. F., et al. (1999). Stages of change across ten health risk behaviors for older adults. Gerontologist, 39, 473-482.

Nisbett, R. E., \& Wilson, T. D. (1977). Telling more than we can know: verbal reports on mental processes. Psychological Review, 84, 231-259.

Nitzke, S., Auld, G., McNulty, J., Bock, M., Bruhn, C.r Gabel, K., et al. (1999). Stages of change for reducing fat and increasing fiber among dietitians and adults with a diet-related chronic disease. Jourtal of the Americen Dietetic Association, 99, 728-731.

Norman, G. J., Velicer, W. F., Fava, J. L., \& Prochaska, J. O. (1998). Dynamic typology clustering within the stages of change for smoking cessation. Addictine Behaviors, 23, 139-153.

Norman, G. J. Velicer, W. F. Fava, J. L., \& Prochaska, J. O. (2000). Cluster subtypes withün stage of change in a representative sample of smokers. Addictive Behaniors, 25, 183-204.

Nosek, B. A, Banaji, M. R., \& Greenwald, A. G. (2002). E-research: ethics, security, design and control in psychological research on the Internet. Journal of Social Issues, 58, 161-176. 
OBrien, T. (1971). Stages of consumer decision making. Jowmal of Marketing Restarch, 8, 283-289.

Ogden, I. (2003). Some problems with socall cognition models: a pragmatic and conceptual analysis. Health $p_{\text {Sychology }} 22,424-4.28$.

Olson, J. M., \& Maio, G. R. (2003). Attitudes in social behavior. In T. Millon \& M. J. Lemer (Eds.), Handbook of psychology: Personality and social psychology (Vol. 5, pp. 299-325). New York: John Wiley \& Sons, lne.

Padula, C. A. Rossi, S., Nigg, C. R., Lees, F., Fey-Yensan, N., Greene; G., al, (2003). Using focus groups for instrument development: application of the transtheoretical Model to fruit and vegetable behaviors of older adults. Journal of Nutrition for the Elderly, 22, 13-33.

Paolini, M., Sapone, A., Canistro, D., Antonelli, M. A. \& Chieco, P. (2003). Diet and risk of cancer. Lancet, 361, 257-258.

Patrician, P. A. (2004). Single-item graphic representation scales. Nursing Research, 53, 347-352.

Perz, C. A., DiClemente, C. C., \& Carbonari, J. P. (1996). Doing the right thing at the right time? The interaction of stages and processes of change in successful smoking cessation. Health Psychology, 15, 462-468.

Pesa, I. A., \& Turner, L. W. (2001). Fruit and vegetable intake and weight-control behaviors among US youth. American Journal of Hewith Behravior, 25, 3-9.

Pill, J. (1971). The Delphi method: substance, context, a critique and an annotated bibliography. SocioEconomic Plamming Science, 5, 57-71.

Plotnikoff, R. C. Hotz, S. B., Birkett, N. I. \& Courneya, K. S. (2001). Exercise and the Transtheoretical Model: A longitudinal test of a population sample. Preventive Medicine, 33, 441-452.

Plummer, B. A., Velicer, W. F., Redding, C. A., Prochaska, J. O., Rossi, J. S., Pallonen, U. E., et al. (2001). Stage of change $e_{x}$ decisional balance and temptations for smoking. Measurement and validation in a large, school-based population of adolescents. Addictive Behnviors, 26, 551571.

Popper, K. R. (1959). The logic of scientific discovery. New York: Basic Books.

Povey, R. Conner, M., Sparks, P.r James, R. \& Shepherd, R. (1999). A critical examination of the application of the transtheoretical Model's stages of change to dietary behaviours. Health Education Research, 14, 641-651.

Prochaska, I. O. (1994). Strong and weak principles for progressing from precontemplation to action on the basis of twelve problem behaviors. Health Psychology, 13, 47-51.

Prochaska, J. O. (2000). Change at different stages. In C. R. Snyder \& R. E. Ingram (Eds.), Handbook of psychological change: psychotherapy processes \& practices for the 21st century. New York: Wiley.

Prochaska, I. O. DiClemente, C., Velicer, W. F., \& Rossi, J. S. (1993). Standardized, individualized, interactive, and personalized self-help programs for smoking cessation. Health $P_{s y c h o l o g y}$ $12,399-405$.

Prochaska, J. O., \& DiClemente, C. C. (1983). Stages and processes of self-change of smoking: Toward an integrative model of change. Journal of Consulting and Chinical Pychology, 51, $390-395$.

Prochaska, J. O., \& DiClemente, C. C. (1998). Comments, criteria, and creating better models. In response to Davidson. In W. R. Miller \& N. Heather (Eds.), Treating addiction behtowiors (2nd ed. $\mathrm{pp}$. 39-45). New York: Flenum.

Prochaska, J. O., DiClemente, C. C., Norcross, J. C. 1992). In search of how people change: Applications to addictive behayiors. Anerican Psychologist, 47, 1102,1114.

Prochaska, J. O., DiClemente, C. C., \& Norcross, J. C. (2003). lin search of how people change: applications to addictive behaviors. In P. Salovey \& A. J. Rothman (Eds.), Socisl psychology of heallh: key readings (pp. 63-77). New York: Psychology Press.

Prochaska, J. O., DiClenente, C. C., Velicer, W. F. \& Rossi, J. S. (1992). Comments on Davidson's "Prochaska and DiClemente's model of change: a case study?" Criticisms and concerns of the transtheoretical model in light of recent research. British journat of Addictiow, 87, 825-835.

Prochaska, J. O., Redding, C. A. \& Evers, K. E. (2002). The transtheoretical model and stages of

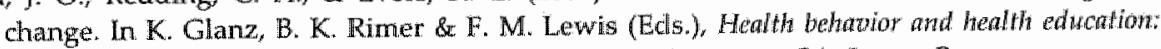
theory, research, and practice (3rd ed., pp. 99-120). San Francisco, CA: Jossey-Bass.

Prochaska, J. O. \& Velicer, W. F. (1997a). The Transtheoretical Model of health behavior change. Annerican Journal of Heal th Promotion, $12,38-48$. 
Prochaska, $), 0$, \& Velicer, W. F. (1997b). Response: misinterpretations and misapplications of the Transitheoretical model. American youmal of Heal th Promotiont, 12, 11-12.

Prochaska, J. O, Velicer, W. F., DiClemente, C. C., \& Fava, J. (1988). Measuring processes of change: applications to the cessation of smoking. Jowmal of Consulting and Clinical Psychology, 56, 520528.

Prochaska, J. O., Velicer, W. F, Fava, J. L., Rossi, J. S., Ksoh, J. Y. (2001). Evaluating a populationbased recuitment approach and a stage-based expert system intervention fot smoking cescation. Addictive Behawiors, 26, 583-602.

Prochaska, J. O., Velicer, W. F, Guadagnoli, E, $\mathbb{R}$ Rossi;, J. S., \& DiClemente, C. (1991). Patterns of Change: Dynamic typology applied to smoking cessation. Multioariate Behavioral Research, $26,83-107$.

Prochaska, J. O, Velicer, W. F., Rossi, J. S, Goldstein, M. G., Marcus, B. H., Rakowski, W., et al. (1994).

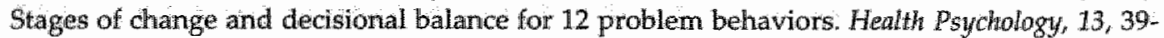
46.

Quinlan, K. B., \& McCaul, K. D. (2000). Matched and mismatched interventions with young adult smokers: testing a stage theory. Health Psychology, 19, 165-171.

Rakowski, W., Ehrich, B., Goldstein, M. G., Rimer, B. K, Pearlman, D. N., Clark, M. A., et al. (1998). Increasing mammography among women aged $40-74$ by use of a stage-matched, tailored intervention. Preventive Medicine, 27, 748-756.

Redding, C. A, Prochaska, J. O., Pallonen, U. E., Rossi, J. S., Velicer, W. F., Rossi, S. R., et al. (1999). Transtheoretical individualized multimedia expert systems targeting adolescents' health behawior. Cognitive and Behavioral Practice, 6, 144-153.

Reed, G., Velicer, W. F., Prochaska, I. O., Rosisi, J. S., \& Marcus, B. H. (1997). What makes a good staging algorithm: examples from regular exercise. Antericam Journal of Health Promotion, 12, $57-66$.

Resnicow $\mathrm{K}_{\text {, }}$ McCarty, F* \& Baranowski, T. (2003). Are precontemplators less likely to change their dietary behavior? A prospective analysis. Health Education Research, 18, 693-705.

Riemsma, R. P., Pattenden, J., Bridle, C. Sowden, A. J., Mather, L., Watt, I. S., et al. (2003). Systematic review of the effectiveness of stage based interventions to promote smoking cessation. British Medical Journal, 326, 1175-1181.

Riva, $\mathrm{G}_{n,}$ Teruzzi, $\mathrm{T}_{t}$ \& Anolli, $\mathrm{L}_{*}$ (2003). The use of the Internet in psychological research: comparison of online and offline questionnaires. Cyberpsychology \& Behowior, 6, 73-81.

Rogers, R. W. (1975). A protection motivation theory of fear appeals and attitude change. Jowrnal of Psychology, 91, 93-114.

Rolls, B. J", Ello-Martin, J. A., \& Carlton Tohill, B. (2004). What can intervention studies tell us about the relationship between fruit and vegetable consumption and weight management. Nutrition Revieus, 62, 1-17.

Roncla, G., Van A.ssema, P., Brugg I. (2001). Stages of change, psychological factors and awareness of pliysical activity levels. Health Promotion Intemational, 16, 305-314.

Rosen, C. 5. (2000). Ils the sequencing of change processes by stage consistent across heallth problems? A meta-analysis. Health Psychology, 19, 593-604.

Rossi, S. R., Rossi, J. S, Rossi-DelPrete, L. M., Prochaska, J. O., Banspach, S. W., \& Carleton, R. A. (1994). A processes of change model for weight-control for participants in community-based weight loss programs. The International Jourtal of the Addictions, 29, 161-177.

Rothiman, A. ). (2004). "Is there nothing more practical than a good theory?": why innowations and advances in health behavior change will arise if interventions are used to test and refine theory. International Journal of Behawional Nutrition and Plysical Activity. 1, doi: 10.1186/1479. 5868-1181-1111.

Rowe, G., Wright, G. \& Bolger, F. (1991). Delphi: a reevaluation of research and theory. Technological Forecasting and Social Change, 39, 235-251.

Rubak, S., Sandbaek, A., Laturitzen, T., \& Christensen, B. (2005). Motivational interviewing: a systematic review and meta-analysis. British Youmal of Genteral Practice, 55, 305-312.

Rychlak, y. (1968). A philosophy of science for personality theory. Boston: Houghton Mifflin. 
Santi, S. M. Cargo, M., Brown, K. S., Best, J. A., \& Cameron, R. (1994). Dispositional risk factors for smoking-stage transitions: A social influences program as an effective modifier. Adalictiwe Behatiors, 19, 269-285.

Sarkar, S. (Ed.). (1996). The emergence of logical empiricism: from 1900 to the Vienn Circle. New York: Garland.

Schumann, A., Meyer, C, Rumpf, H. J., Hapke, U., \& John, U. (2002). Naturalistic changes in the readiness to quit tobacco smoking in a German general population sample. Prewentive Medicine, 35, 326-333.

Schwarzer, R. (1992a). Selfefficacy: Thought control of action. Washington: Hemisphere Pub. Corp.

Schwarzer, R. (1992b). Self-efficacy in the adoption and maintenance of health behaviors: Theoretical approaches and a new model. In R. Schwarzer (Ed.), Self-efficacy: Thought control of action (pp. 217-243). London: Hemisphere.

SCP. (2004). In het zicht van de toekonst. Socian en Cultureel rapport 2004 (In view of the futwre: social and cultural report 2004]. Den Haag: Sociaal en Cultureel Planbureau.

Segan, C. J., Borland, R., \& Greenwood, K. M. (2002). Do transtheoretical model measures predict the transition from preparation to action in smoking cessation? Psychology and Health, 17, 417435.

Segan, C. J., Borland, R., Greenwood, K. M. (2004). What is the right thing at the right time? Interactions between stages and processes of change among smokers who make a quit attempt. Health Psychology, 23, 86-93.

Serdula, M. K., Gillespei, C., Kettel-Khan, L., Farris, R., Seymour, J., \& Denny, C. (2004). Trends in fruit and vegetable consumption among adults in the United States: behavioral risk factor surveillance system, 1994-2000. American Journal of Public Health, 94, 1014-1018.

Sheeran, P. (2002). Intention-behaviour relations: A conceptual and empirical review, European Review of Social Psychology, 12, 1-36.

Sheeran, $P_{.}$\& Abraham, C. (2003). Mediator of moderators: temporal stability of intention and the intention-behavior relation. Personality and Social Psychology Bulletin, 29, 205-215.

Sheeran, P. Webb, T. L., \& Gollwitzer, P. M. (2005). The interplay between goal intentions and implementation intentions. Personality and Social Psychology Bulletin, 31, 87-98.

Siegel, $S_{\text {, \& }}$ Castellan, N. J. (2002). Nonparametric statistics for the behavioral sciences (2nd ed.). New York: McGraw-Hill.

Siero, F. W., Broer, J., Bemelmans, W. J. E., \& Meyboom-de Jong, B. M. (2000). Impact of group nutrition education and surplus value of Prochaska-based stage-matched information on health-related cognitions and on Mediterranean nutrition behavior. Health Education Research, 15, 635-647.

Smedslund, G. (1997). Some psychological theories are not empirical: A conceptual analysis of the "stages of change" Model. Theory \& Psychology, 7, 529-544.

Srinath Reddy, K., \& Katan, M. B. (2004). Diet, nutrition and the prevention of hypertension and cardiovascular diseases. Public Health Nutrition, 7, 167-186.

Statistics Netherlands. (2003). Statistisch Jaarboek 2003 [Anmal statistical report 2003]. Voorburg/Heerlen: Centraal Bureau voor de Statistiek.

Sutton, S. (1998). Predicting and explaining intentions and behavior: how well are we doing? fournal of Applied Social Psychology, 28, 1317-1338.

Sutton, S. (2000a). A critical review of the transtheoretical Model applied to sinoking cessation. In P. Norman, C. Abraham \& M. Conner (Eds.), Understanding and changing health behaviow: from health beliefs to self-regulation (Pp. 207-225). Amsterdam: Harwood Academic Publishers.

Sutton, S. (2000b). Interpreting cross-sectional data on stages of change. Psychology and Health, 15, 163171 .

Sutton, S. (2001). Back to the drawing board? A review of applications of the transtheoretical modiel to substance use. Addiction, 96, 175-186.

Swamborn, P. G. (1987). Methoden wan sociaal-wetenschappelijk onderzoek [methods in social sciencel. Meppel: Boom.

Swinburn, B., Egger, G., \& Raza, F. (1999). Dissecting obesogenic environments: the developmerit and application of a framework for identifying and prioritizing environmental interventions for obesity. Preventive Medicine, 29, 563-570. 
Topp, N. W, \& Pawloski, B. (2002), Online data collection. Journal of Science Edwcation and Technology, $11,173-178$.

Trichopoulou, A. \&agiou, P. (1997). Healthy traditional Mediterranean diet: An expression of culture, history, and lifestyle. Nutrition Reoiews, $55,383-389$.

Trudeau, E., Kristal, A. R., Li, S, \& Patterson, R. E. (1998). Demographic and psychosocial predictors of fruit and vegetable intakes differ: implications for dietary interventions. Joumal of the Antericun Dietetic Association, 98, 1412-1417.

Vallis, M., Ruggiero, L., Greene, $G_{*,}$ Jones, H., Zinman, B., Rossi, $S$, et al. (2003). Stages of change for healthy eating in diabetes. Diabietes Care, 26, 1468-1474.

Van Assema, P., Brug, J., Ronda, G., Steenhuis, I., \& Oenema, A. (2002). A short Dutch questionnaire to measure fruit and vegetable intake: relative validity among adults and adolescents. Nutrition and Health, 16, 85-106.

Van der Wilk, E. A. \& Jansen, J. (2005). Lifestyle-related risks: are trends in Europe converging? Public Health, 119, 55-66.

Van Duyn, M. A., Kristal, A. R., Dodd, K., Campbell, M. K., Subar, A. F., Stables, G., et al. (2001). Association of awareness, intrapersonal and interpersonal factors, and stage of dietary change with fruit and vegetable consumption: A national survey. American Journal of Health Promotion, $16,69-78$.

Van Duyn, M. A. S., Heimendinger, I., Russek-Cohen, E., DiClemente ${ }_{*}$ C. C. Sims, $_{\text {, }}$. S., Subar, A. F, et al. (1998). Use of the transtheoretical model of change to successfully predict fruit and vegetable consumption. Journal of Nutrition Education and Behavior, 30, 371-380.

Van Oers, J. A. M. (2002). Gezondheid op koers? Volksgezondheid Toekonst Verkenning 2002 [Health on course? The 2002 Dutch public health status and farecasts reportl. Bilthoven: RIVM.

Van Sluijs, E. M. F, Van Poppel, M. N. M., \& Van Mechelen, W. (2004). Stage-based lifestyle interventions in primary care: are they effective? American Journal of Preventive Medicine, 26, 330-343.

Velicer, W. F., DiClemente, C. C., Prochaska, J. O., \& Brandenburg, N. (1985). Decisional balance measure for assessing and predicting smoking status. Journal of Personality and Social Psychology, 48, 1279-1289.

Velicer, W. F., DiClemente, C. C., Rossi, J. S., \& Prochaska, J. O. (1990). Relapse situations and selfefficacy: an integrative model. Addictive Behaniors, 15, 271-283.

Velicer, W. F, Hughes, S. L., Fava, J. L., \& Prochaska, J. O. (1995). An empirical typology of subjects within stage of change. Addictive Behaviors, 20, 299-320.

Velicer, W. F., Martin, R. A., Collins, L. M. (1996). Latent transition analysis for longitudinal data. Addiction, 91, S197-S209.

Velicar, W. F., Norman, G. J., Fava, I. L., \& Prochaska, I. O. (1999). Testing 40 predictions from the transtheoretical model. Addictive Behaviors, 24, 455-469.

Velicer, W. F., Prochaska, J. O., Fava, J., Norman, G. J., Redding, C. A. (1998). Smoking cessation and stress management: Applications of the Transtheoretical Model of behavior change [Electronic version]. Honneostasis, 38, 216-233.

Velicer, W. F, Rossi, J. S, Diclemente, C. C., \& Prochaska, J. O. (1996). A criterion measurement model for health behavior change. Addictioe Behaviors, 21,555-584.

Weinstein, N. D. (1988). The precaution adoption process. Health Psychology, 7, 355-386.

Weinstein, N. D. (1993). Testing four competing theories of health-protective behavior. Health Psychology, 12, 324-333.

Weinstein, N. D., Rothman, A. J., Sutton, S. R. (1998). Stage theories of health behavior: Conceptual and methodological issues. Health Psychology, 17, 290-299.

Weinstein, N. D., \& Sandman, P. M. (1992). A model of the precaution adoption process: evidence from home radon testing. Health Psychology, 11, 170-180.

WHO/FAO. (2003). Diet, nutrition and the prevention of chronic diseases. Geneva: World Health Organisation. 


\section{Summary \& Samenvatting}


3

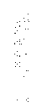

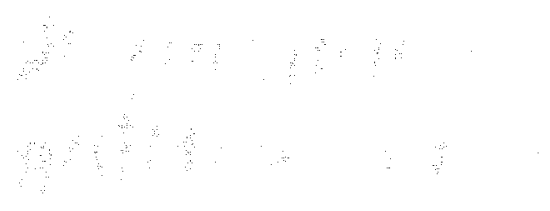




\section{Summary}

The Transtheoretical model (TTM) is a stage model of behavior change, which is widely applied to study determinants of health behaviors, to develop interventions and to evaluate the impact of such interventions. The TTM construes behavior change as a dynamic process involving movement through a series of five so-called stages of change: precontemplation (no intention to change behavior within the next six months), contemplation (intention to change behavior within the next six months), preparation (intention to change behavior within 30 days), action (people changed from unhealthy to healthy behavior within the past six months), and maintenance (maintenance of the behavior change for more than six months). According to the TTM, behavior change is not a linear process, but a discontinuous process; people tend to cycle through these different stages. People can regress (or relapse) to all earlier stages of change, but, when people progress, it is assumed that individuals will pass through all the stages as outlined by the TTM and stage skipping is not expected. Such movements between stages of change are called stage transitions. In addition to stages of change, the TTM describes factors that can facilitate such stage transitions, i.e., decisional balance, self-efficacy and processes of change.

Although the TTM is widely applied, debate exists about the model's validity. Four criteria have been proposed which can discern a sound stage model from a pseudostage (a model that segments an underlying continuum into categories) or continuum model of behavior change. First, a stage theory needs a set of rules that assign each individual to one of a limited number of categories, a so-called classification system to define the stages. Second, a stage model should specify the sequence of stages through which a substantial majority of people moves towards lasting behavior change. Third, people at a given stage face similar barriers and can thus be helped by similar interventions. Fourth, people in different stages face different barriers and thus at each stage different interventions are needed to help people progress through the stages. Following from the third and fourth criteria, stages of change should be qualitatively different.

This thesis presents studies that systematically explore these assumptions and predictions of the TTM when applied to fruit intake. The thesis consists of two parts. Part 1 focuses on predictors of stage transitions (chapters 1-3), whereas part 2 examines whether health behavior change is best described by stages of change (chapters 4-6).

In chapter 1 the results of a three-round Delphi study among stages of change researchers are presented. The Delphi study was conducted to make an inventory of opinions and examine agreement on determinants of forward transitions between Transtheoretical stages of change. In the first round, ten experts completed an electronic questionnaire with open-ended questions about 
potential determinants for each stage transition. In the second round, a structured electronic questionnaire based on the first round results, was sent to authors of scientific papers on stages of change, published between 1995 and May 2002. In the third round, participants were presented feedback about the second round and were asked to re-rate their answers based on the information provided. Resulls showed that participants agreed on various transition determinants, but that determinants were not always stage-specific, e.g. control-related issues and social support were identified as determinants of all transitions from contemplation to maintenance. The results further showed lower consensus about determinants of earlier stage transitions than about determinants of later stage transitions. Factors that are associated with these earlier stage transitions were tested in chapters 2 and 3.

In chapter 2 the cross-sectional and longitudinal associations between decisional balance, self-efficacy, fruit intake, and stage of change transition out of precontemplation and contemplation were tested in a longitudinal cohort study among 735 adults using electronic questionnaires. Two equal time intervals of 53 days were analyzed longitudinally and three points in time cross-sectionally. Pros, fruit intake and self-efficacy predicted forward stage transition from precontemplation. Self-efficacy also predicted forward stage transition from contemplation. Cons did not predict stage transitions. Results from longitudinal analyses were similar to cross-sectional analyses, except for self-efficacy; no crosssectional differences between early stages in self-efficacy were found. Consistent with the TTM, self-efficacy and pros were relevant for stage transitions, although these predictors were not always be stage-specific.

The role of the Transtheoretical processes of change in stage transitions is presented in chapter 3 . The design employed was identical to the design used in chapter 2 . The processes of change represent the overt and covert activities that people use to progress from stage to stage. Two types of processes of change exist, i.e., experiential (cognitive, affective and evaluative processes) and behavioral (active strategies) processes. Cross-sectional differences were found for the processes of change between precontemplation and all further stages. Experiential as well as behavioral processes increased from precontemplation to action with similar patterns. Both experiential and behavioral processes predicted forward transition out of precontemplation and forward transition into action, while only behavioral processes predicted forward transition out of contemplation. More frequent application of processes of change seemed associated with more advanced stages of change, although the two-factor structure of experiential and behavioral processes may not be distinguishable for fruit intake.

Chapter 4 examines stability and transitions in Transtheoretical stages of change for fruit intake. Six assessments took place within a four-month period with varying time intervals using electronic questionnaires. Precontemplation and maintenance were the most stable stages of change, while preparation was 
the least stable. Longer time intervals showed lower stability, but frequent stage transitions also occurred in shorter time intervals. Seven transition profiles were distinguished, e.g. Progress, Regress then Progress, or Multiple Change. Sequences within transition profiles mostly showed stage transitions to adjacent stages, independent of the interval duration. Differences in fruit intake, age, level of education and ethricity were found between different transition profiles. Stability rates and most transition sequences largely support the Transtheoretical Model, although the results might also support a pseudostage model of behavior change.

In chapter 5 it is shown that the pre-action Transtheoretical stages of change applied to fruit intake in fact may reflect such pseudostages, i.e., a stagelike categorization of a continuous behavioural intention scale. Dichotomizing the continuous intention measure created an unmotivated pseudostage (neutral or negative intention) and a motivated pseudostage (positive intention). These were compared to the precontemplation and contemplation/preparation stages of change. Strong associations were found between pseudostages and stages of change. Second, for a majority of respondents, stability and transitions in stages of change resembled transitions in pseudostage, while test-retest reliabilities for both measures were similar. Finally, stages and pseudostages both predicted fruit intake, but in a regression model with both measures, only pseudostages contributed significantly to the prediction of fruit intake. This might indicate that stages and pseudostages explain the same part of the variance in fruit intake.

Further indications that the Transtheoretical stages of change may not be valid when applied to fruit intake are presented in chapter 6 . In a so-called match-mismatch test, precontemplators and contemplators were randomly assigned to receive web-based individualized precontemplation-matched, contemplation-matched or action-matched fruit promotion feedback. Precontemplators receiving the action-matched feedback had a marginally significantly higher fruit intake compared to those in the precontemplationmatched condition, but no differences were found in intention and stage progression. Contemplators in the precontemplation-matched condition had higher intentions than contemplators in the contemplation-matched condition, but no differences between the conditions were found for fruit intake and stage progression. The results described in chapter 6 failed to show superiority of stagematched information in the promotion of fruit intake.

In the final chapter, the general discussion, the results are discussed and integrated. Further, methodological and theoretical issues are discussed, as well as implications for future research and practice. We conclude that although the TTM might still have pragmatic value in describing health behavior change, the studies presented showed that the TTM might not be a valid stage model for fruit intake. 


\section{Samenvatting}

Het Transtheoretisch model (TTM) beschrijft gedragsverandering als een dynamisch proces, waarbij een serie van vijf fasen wordt doorlopen. In de precontemplatie fase zijn mensen niet van plan om binnen zes maanden het gedrag te veranderen. In contemplatie zijn mensen wel van plan om binnen zes maanden het gedrag te veranderen, terwijl in preparatie mensen al op korte termijn, binnen één maand, hun gedrag willen aanpassen. De actie fase wordt gekenmerkt door een zichtbare gedragsverandering die ergens in de laatste zes maanden gemaakt is, zoals bijvoorbeeld het stoppen met roken. Wanneer deze verandering langer dan zes maanden wordt volgehouden, bereikt men de behoud fase. Gedragsverandering is geen lineair proces, maar eerder cyclisch. Mensen gaan zowel vooruit als achteruit door de verschillende fasen. Het TTM veronderstelt dat fasen niet worden overgeslagen, wanneer mensen door de fasen vooruit gaan. Wanneer mensen van de ene fase naar een andere fase gaan wordt dit transitie genoemd. Naast de fasen van verandering, beschrijft het TTM ook een aantal factoren die transities door de fasen zouden vergemakkelijken, zoals de decisional balance (de afweging van voor- en nadielen van een bepaald gedrag), eigen-effectiviteit (inschatting van de eigen vaardigheden) en processen van verandering (strategieën die mensen inzetten om vooruit te gaan).

Ondanks dat er veel discussie bestaat over de validiteit van het TTM, wordt dit model vaak gebruikt om determinanten van gezondheidsgedrag in kaart te brengen, ${ }_{r}$ om interventies mee te ontwikkelen en om de effectiviteit van dergelijke interventies te evalueren. Er bestaan vier criteria waaraan het TTM zou moeten voldoen om valide te zijn. Ten eerste moeten mensen aan één van de verschillende fasen toegewezen kunnen worden. Ten tweede zou het TTM moeten definiëren welke volgorde van de fasen door een grote meerderheid van de mensen gevolgd wordt. Ten derde ervaren mensen in dezelfde fase dezelfde barrières tot gedragsverandering. Hierdoor zullen zil ook van dezelfde interventies profiteren. Ten vierde ervaren mensen in verschillende fasen verschillende barrières tot gedragsverandering. Er zijn dus voor elke fase verschillende interventies nodig.

In dit proefschrift worden systematisch deze verschillende aannames en voorspellingen van het TTM getoetst, toegepast op fruitconsumptie. Het eerste deel concentreert zich op voorspellers van bewegingen tussen de fasen, zogenaamde transities (hoofdstuk 1-3). In het tweede deel wordt bekeken of gedragsverandering wel volgens fasen verloopt zoals verondersteld door het TTM (hoofdstuk 4-6).

In hoofdstuk 1 worden de resultaten van een Delphi studie gepresenteerd. Deze studie werd uitgevoerd onder onderzoekers die tussen 1995 en mei 2002 gepubliceerd hebben in peer-reviewed literatuur over fasenmodellen van 
gedragsverandering. De Delphi studie bestond uit drie ronden, waarbij het doel was een overzicht van meningen te genereren en consensus over determinanten van transities tussen fasen in kaart te brengen. De resultaten lieten zien dat deelnemers het over verscheidene transitiedeterminanten eens waren, maar ook dat de determinanten niet altijd stage-specifiek waren. Bijvoorbeeld, sociale steun en determinanten gerelateerd aan controle werden geïdentificeerd als determinanten van alle transities tussen contemplatie en behoud. De resultaten lieten verder zien dat er minder consensus bestond over determinanten van transities tussen eerdere fasen in het model (van precontemplatie naar preparatie) dan over determinanten van latere transities (van preparatie naar behoud).

In hoofdstuk 2 worden de cross-sectionele en longitudinale associaties tussen de decisional balance, eigen-effectiviteit, fruitconsumptie en voorwaartse transitie vanuit precontemplatie en contemplatie getoetst in een longitudinale cohort studie onder 735 volwassen. Data werden verzameld met behulp van elektronische vragenlijsten. Drie meetmomenten werden cross-sectioneel geanalyseerd en twee gelijke tijdsintervallen van 53 dagen tussen deze momenten werden longitudinaal geanalyseerd. Meer voordelen, hogere fruitconsumptie en hogere eigen-effectiviteit voorspelden dat mensen vanuit precontemplatie vooruit gingen. Hogere eigen-effectiviteit voorspelde dat mensen vanuit contemplatie vooruit gingen. De longitudinale analyses leverden vrijwel dezelfde resultaten als de cross-sectionele analyses, behalve voor eigen-effectiviteit. Er werd crosssectioneel geen verschil in eigen-effectiviteit aangetoond tussen precontemplatie, contemplatie en preparatie.

Consistent met het TTM, bleken eigen-effectiviteit en de voordelen van fruitconsumptie relevant te zijn voor transities. Tegenstrijdig aan het TTM, bleken nadelen van fruitconsumptie niet van belang voor transities tussen de fasen en bleek eigen-effectiviteit geen fase-specifieke rol te spelen.

De rol van de Transtheoretische processen van verandering in transities wordt gepresenteerd in hoofdstuk 3, waarbij de opzet van de studie gelijk was aan de opzet in hoofdstuk 2 . De processen van verandering geven de strategieên weer die mensen gebruiken om van de ene fase naar een volgende fase te gaan. Het TTM onderscheidt twee soorten processen, de cognitieve (de denkprocessen) en de gedragsmatige (de doeprocessen). Er werden cross-sectionele verschillen in processen van verandering gevonden tussen precontemplatie en alle andere fasen. De toepassing van cognitieve en gedragsprocessen nam toe vanaf precontemplatie tot aan actie, waarbij beide soorten processen een gelijk patroon volgden. Zowel cognitieve als gedragsprocessen voorspelden dat mensen vanuit precontemplatie vooruit gingen en dat mensen naar actie gingen. De gedragsprocessen, maar niet de cognitieve processen, voorspelden dat mensen vanuit contemplatie vooruit gingen. Consistent met het TTM bevonden mensen die de processen van verandering vaker gebruiken zich vaker in verdere fasen van verandering. De resultaten lieten echter ook zien dat cognitieve en 
gedragsprocessen niet zo duidelijk te onderscheiden zijn voor fruitconsumptie en dat de processen geen fase-specifieke rol speelden.

In hoofdstuk 4 wordt getracht inzicht te krijgen in stabiliteit en transities van de Transtheoretische fasen van verandering voor fruitconsumptie. In een periode van vier maanden werden op zes meetmomenten elektronische vragenlijsten afgenomen. Het tijdsinterval tussen de metingen varieerde: Precontemplatie en behoud waren de meest stabiele fasen. Preparatie was de minst stabiele fase. De fasen van verandering leken minder stabiel te zijn naarmate het tijdsinterval tussen de metingen groter was, alhoewel transities ook frequent voorkwamen in kortere tijdsintervallen. Zeven transitieprofielen werden onderscheiden, zoals Progress (mensen die alleen vooruit gaan gedurende vier maanden) en Regress then Progress (mensen die na een terugval weer vooruitgaan door de fasen). Binnen deze transitieprofielen gingen respondenten die van fase veranderden met name naar aangrenzende fasen (bijvoorbeeld van precontemplatie naar contemplatie), ongeacht het tijdsinterval. De transitieprofielen bleken te verschillen met betrekking tot fruitconsumptie, leeftijd, opleidingsniveau en etniciteit. Stabiliteit en transities naar aangrenzende fasen ondersteunen het TTM, maar zouden eveneens consistent zijn met een continu model van gedragsverandering.

In hoofdstuk 5 is verder onderzocht of het TTM een fasen model of continu model van gedragsverandering weergeeft. Het is mogelijk dat het TTM fasen onderscheidt die eigenlijk categorieën binnen een continuüm zijn, zogenaamde pseudofasen. Door een een continue intentiemaat te dichotomiseren werd een ongemotiveerde pseudo-fase (neutrale of negatieve intentie) gecreeërd, vergelijkbaar met de precontemplatie fase en een gemotiveerde pseudo-fase (positieve intentie) vergelijkbaar met de contemplatie/preparatie fasen. De resultaten leken er inderdaad op te wijzen dat de Transtheoretische fasen van verandering toegepast op fruitconsumptie in feite pseudo-fasen reflecteren. Ten eerste werden er sterke associaties gevonden tussen fasen en pseudo-fasen van verandering. Ten tweede kwamen voor een meerderheid van de respondenten transities en stabiliteit in fasen en pseudo-fasen overeen en was de test-hertest betrouwbaarheid van beide maten ongeveer gelijk. Ten derde voorspelden zowel fasen als pseudo-fasen fruitconsumptie, maar wanneer beiden werden opgenomen in een regressiemodel voorspelde alleen pseudo-fasen de fruitconsumptie. Dit kan er op duiden dat zowel fasen als pseudo-fasen dezelfde variantie in fruitconsumptie verklaren.

Verdere aanwijzingen dat de Transtheoretische fasen van verandering wellicht niet valide zijn, worden beschreven in hoofdstuk 6 . In een zogenaamde match-mismatch test werden respondenten in precontemplatie en contemplatie aselect toegewezen aan web-based advies op maat dat was afgestemd op determinanten die volgens het TTM relevant zijn voor precontemplatie, contemplatie of actie. Precontemplatoren die advies kregen over actie-relevante 
factoren hadden een marginaal hogere fruitconsumptie dan precontemplatoren die advies kregen afgestemd op precontemplatie-relevante factoren. Er werden geen verschillen gevonden tussen de drie adviezen in intentie of voorwaartse transitie voor precontemplatoren. Contemplatoren die advies kregen over de precontemplatie factoren hadden een sterkere intentie om twee stuks fruit per dag te eten dan contemplatoren die advies ontvingen over factoren die relevant zouden moeten zijn voor contemplatie. Voor contemplatoren verschilden de drie adviezen niet in effectiviteit voor fruitconsumptie en voorwaartse transitie. De veronderstelling van het TTM dat advies afgestemd zou moeten worden op de fasen van verandering werd niet bevestigd in het experiment gericht op fruitconsumptie.

In het laatste hoofdstuk van dit proefschrift, de algemene discussie, worden de resultaten van de verschillende studies tenslotte geintegreerd en bediscussieerd. Tevens worden in dit hoofdstuk methodologische en theoretische aspecten van het onderzoek bediscussieerd, evenals implicaties voor toekomstig onderzoek en aanbevelingen voor de praktijk. De conclusie is dat het TTM wel voor pragmatische doeleinden gebruikt kan worden, bijvoorbeeld in de beschrijving van gezondheidsgedrag. De validiteit van het TTM is echter niet aangetoond. 


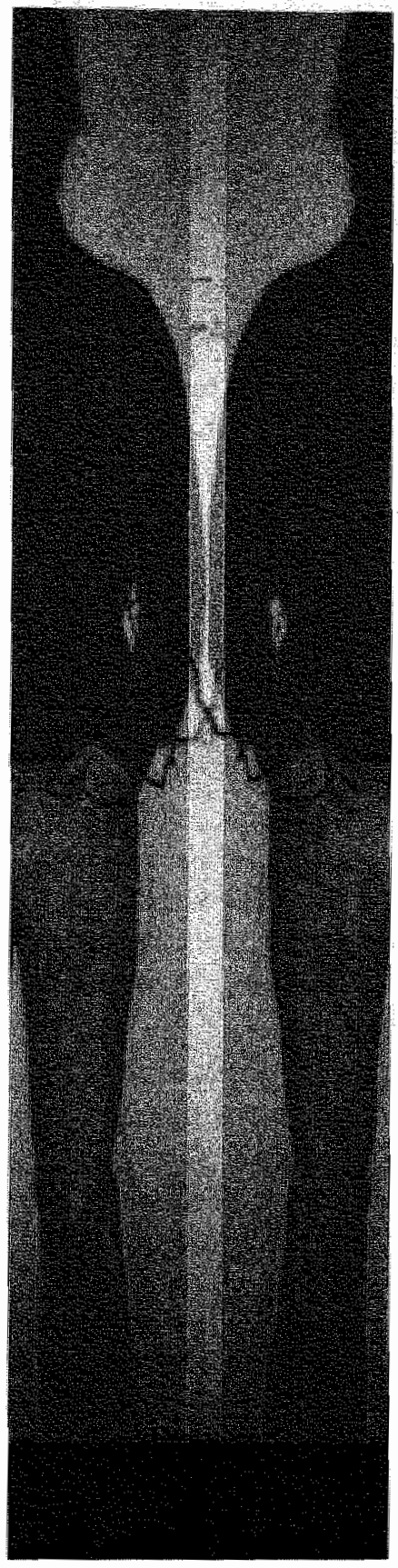

\section{Acknowledgements \& Curriculum Vitae}


b t

$\vdots$

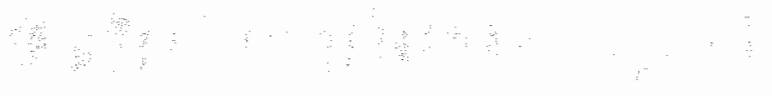




\section{About the author}

Emely de Vet was born on May 19, 1978 in Oud Gastel, the Netherlands. In 1996 she received a VWO diploma at the Norbertuscollege in Roosendaal. In that same year she started university education in Health Education and Health Promotion at the Faculty of Health Sciences of the Universiteit Maastricht. After graduation in 2001, she traveled around South-East Asia. Afterwards she worked for a short period of time as a researcher at the Department of Health Ethics at the Faculty of Health Sciences of the Universiteit Maastricht, and helped nursing at the psychiatric hospital Vijverdal in Maastricht. In 2002, she started as a PhD student at the Department of Health Education and Health Promotion at the Faculty of Health Sciences of the Universiteit Maastricht on a project entitled 'The validity of the Transtheoretical model'. The studies that were conducted as part of this project resulted in this thesis. Besides conducting research, Emely engaged in teaching and represented the $\mathrm{PhD}$ fellows in the $\mathrm{PhD}$-students council of the Nutrition and Toxicology Research Institute (NUTRIM). From January 2006 onwards, she will work as a post-doc researcher on the impact of implementation intentions in changing complex health-related behavior at the Department of Public Health of the Erasmus University Medical Center in Rotterdam. 


\section{List of publications}

\section{Full papers}

Brugg I., De Vet, E., De Nooijer $f_{r}$., Verplanken, B. (in press). Predicting frutit consumption: cognitions, intention and habits. Joumal of Nutrition Education and Behatior

De Nooijer, I., Van Assema, P., De Vet, E., \& Brug, J. (2005). How stable are stages of change for nutrition behaviors. Heal h Promotion Intermational, 20, 27-34.

De Nooijer, J., De Vet, E., Brug, J. \& De Vries, N. K. (in press). Do implementation intentions help to turn good intentions into higher fruit intakes? Joumal of Nutrition Education and Behuroior.

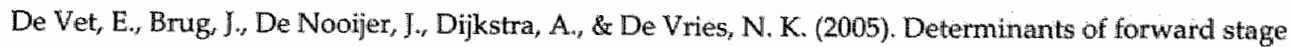
transitions: a Delphi study. Health Education Research, 20, 195-205.

De Vet, E, De Nooijer, J., De Vries, N. K., \& Brug, J. (2005). Determinants of forward stage transition from precontemplation and contemplation for fruit consumption. American Jowrnal of Health Pronotion, 19, 275-285.

De Vet, E., De Nooijer, J., De Vries, N. K. \& Brug, J. (2005). Stages of Change in fruit intake: a longitudinal examination of stability, stage transitions and transition profles. Psychology and Health, 20, 415-428.

De Vet, E., De Nooijer, J., De Wries, N. K., \& Brtg, J. (under review). The Transtheoretical stages of change and fruit intake: real or pseudostages?

De Vet, E., De Nooijer, I. De Vries, N. K., \& Brug, I. (under review). Do the Transtheoretical processes of change predict transitions in stages of change for fruit intake?

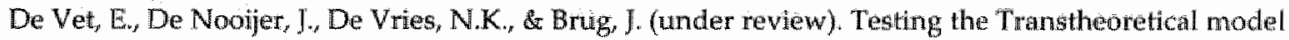
for fruit intake: comparing web-based individualized stage-matched and stage-mismatched feedlback.

Reubsaet, A., Brug, J., De Vet, E, \& Van den Borne, B. (2003). The effects of practicing registration of organ donation preference on self-efficacy and registration intention: an enactive mastery experience. Psychology and Health, 18, 585-594.

\section{Abstracts}

Brug, J, De Vet, E., De Nooijer, J, \& Verplanken, B. (2004, June). Predicting fruit consumption: cognitions, intention and habits. Paper presented at the Third Confenence of the International Society of Behavioral Nutrition and Physical Activity, Washington D.C., US. 
De Nooijer, J., De Vet, E., Brugg, J. \& De Vries, N. K. (2004, May). Impiementation intentions for fruit consumption: tuming good intentions into great results? Paper presented at the Fifth Conference on Psychology and Health, Kerkrade, The Netherlands.

De Nooijer, J, Van Assema, P., De Vet, E., \& Brug, ). (2004, August). How stable are stages of change for nutrition behaviors. Poster session presented at the Conference "Research on the Transtheoretical Model: Where are we now, where are we going?" Marburg, Germany

De Vet, E.W.M.L., Brug I., Dijkstra, A, \& De Vries, N.K. (2002, June). The validity of stages of change: researching the fundamental and practical implications of stages of change applied to fruit and vegetable consumption. Poster session presented at the Xth Food Choice Conference, Wageningen, The Netherlands.

De Vet, Li., De Nooijer J. \& Brug, J. (2003, April). De validiteit van het Transtheoretisch Model. Paper gepresenteerd tijdens het Nederlands Public Health Congres, Amsterdam, Nederland.

De Vet, E, De Nooijer; J. \& Brug, J. (2003, July). Forward stage transition determinants of fruit consumption. Poster session presented at the Second Conference of the International Society of Behavioral Nutrition and Physical Activity, Québec City, Canada.

De Vet, E., De Nooijer, J., De Vries, N. K., \& Brugg, J, (2004, May). Stage transition determinants in fruit intake: Cross-sectional versus longitudinal results. Paper presented at the Fifth Conference on Psychology and Health, Kerkrade, The Netherlands.

De Vet, E., De Nooijer, J., De Vries, N. K., \& Brug, J. (2004, June). Stages of Change in frut intake: a longitudinal examination of stability, stage transitions and transition profiles. Paper presented at the 18 Conference of the European Health Psychology Society, Helsinki, Finland.

De Vet, E., De Nooijer, J., De Vries, N. K, \& Brug, J. (2004, August). How applicable is the Transtheoretical model to fruit intake? Paper presented at the Conference "Research on the Transtheoretical Model: Where are we now, where are we going?" Marburg, Germany.

De Vet, E., De Nooijer, J, De Vries, N. K., \& Brug, J. (2005, June). The validity of the Transtheoretical model applied to iruit intake. In S. Biddle (Chair), Strenghs and linnitations of the stages of change model as applited to diet and physical nctivity. Symposium conducted at the Fourth Conference of the International Society of Behavioral Nutrition and Physical. Activity, Amsterdan, The Netherlands.

De Vet, E., De Nooijer, J., De Vries, N. K., \& Brug, J. (2005, August). Do the Transtheoretical processes of change predict transitions in stages of change for fruit intake? Paper presented at the $19^{\text {th }}$ Conference of the European Health Psychology Society, Galway, Ireland.

De Vet, E., De Nooijer, J., De Vries, N. K., \& Brug, J. (2005, August). The Transtheoretical stages of change and fruit intake: real or pseudostages? Poster session presented at the 19 th Conference of the European Health Psychology Society, Galway, Ireland. 


\section{Acknowledgements}

This dissertation would not have been realized, if I were not so fortunate to have the support of many people in even so many ways. To those who contributed, I would like to express my gratitude. First of all, I owe a great thanks to my supervision team. I call myself lucky to may have experienced such a rich mixture of supervision styles, with Nanne playing the devil's advocate, Hans providing inspiring and critical feedback, and Jascha motivating me with a personal touch all along and outside the project. Jascha, Hans, and Nanne thank you for your trust, for giving me the freedom to develop and work on my own ideas, and for having such a lot of patience with a stubborn phd-student like me. I hope we will continue our cooperation in the future.

I thank the scientific committee for their efforts on reading this thesis: Hein de Vries, Gerjo Kok, Stef Kremers, Denise de Ridder and Neil Weinstein. I thank ZonMW for providing financial support to conduct the studies, and Flycatcher Internet Research, Intomart GfK, their Internet panel members, and the Delphi study experts for contributing to the research.

Many thanks to my colleagues in Maastricht for their (practical) support, but above all for the fun. I really enjoyed my time at the department.

I thank Lydia and Maartje for standing by my side, at many moments more than only during the defense of this thesis. Ellen, thank you for designing the cover of this thesis. Marjolein, Maud, and Mascha, I am thankful that you followed my example and stayed in Maastricht after graduation. I promise that I will visit Maastricht frequently. De jaarclub, 't ouwe huis, Jouke and Rikkie, I will live closer to you all, so be warned.

Pa en Ellie, Eloy en Michael, ik wil jullie bedanken voor jullie geloof in mij, maar ook voor het relativeren van deze scriptie. Ik zal niet naast m'n schoenen gaan lopen.

Rob, as a boyfriend, friend and colleague, you were supportive in incountable ways. It is not an easy job to finish two dissertations under one roof. Even at times you suffered from an aio-dip, you still cared about my progress and helped me out with the, often minor, troubles I faced. I hope we will soon share a roof again. 
The "Kurt Lewin Institute Dissertation Series" started in 1997.

Since 2003 the following dissertations have been published:

2003-1: Femke Bennenbroek: Social comparison and coping witl radiation therapy. The significance of different dimensions of comprarison

2003-2: Marlene Roefs: Public Participation and Perceived (in)justice in Sow th Africh, 1995-2000

2003-3: Rick van Baaren: Minicry: A sacial perspectioe

2003-4: Michaéla Schippers: Reflexiwity in teams

2003-5:

2003-6:

Heleen van Mierlo: Self-managing temuork wh psychological well-being

2003-7:

2003-8:

2003-9:

2004-1:

2004-2:

2004-3:

2004-4:

2004-5: Ruben Taris: Person-Emotronntent Fit: A longitudinal shudy of the interaction betwoun enployees characteristics and work environmentad characteristics

2004-6:

Rob Holland: On the structure and consequences of attitude strength

Camiel Beukeboom: How mood wrons on language

Joost Miedema: Faimess and the Self

Wolfgang Steinel: Misledding in social decision-making: A motiontional approach

Edwin van Hooft: fob seeking as planned behavior. In search of group differences

Kirsten Ruys: The influence of self and similarity on inmediate affection inumessions of people Michel Handgraaf: Self-interest and other-oriented motines in social decision making: An ultimatum bargaining perspective

Sjoerd Goslinga: Betrokkenheid, participatie en ledenverloop in vakbonden

2004-7: Jaap Ham: Bridging attribution and spontaneous inferences: Spontaneous and intertional components of dispositional and situational inferences

2004-8: Mirjam Tazelaar: When things go wrong: The influence of noise on interactions

2004-9: Marjolein de Best-Waldhober: Coordinatic in interdependente situaties: Over de afweging van directe witkansten versus witkomsten op lange bernijn

2004-10: Hinke Groothof: When others are doing better or worse: Responses from the hart and the hend

2004-11: $\quad$ Laetitia Mulder: Throwing light on the dark side of sinctions. Sanctioning systens in social dilemmas re-exanined

2004-12: Annebel de Hoogh: Personality and charismatic lendership: A matter of context

2004-13: Brigitte sen Brink: Psychological controct: A useful concept?

2004-14: Gerben van Kleef: Emotion in social conflict. The interpersonnl effects of ewotions in negofiations

2004-15: Tom Frijns: Keeping secrets: Quantity, quality and consequences

2005-1: Elsbeth Steenland: Stereotype Change: Effects of disconfirming information

2005-2: Annet de Lange: What about causality? Examining longitudinal relations between work characteristics and nental health

2005-3: Reint Jan Renes: Sustained zolunteerism: Justification, mothation and wranagenent

2005-4:

2005-5:

2005-6:

Aloyse Augusta Dinsbach: Socialization in the workplace: A focus on migranf groups

Susarne Peters: The social psychology of being better off than others

2005-7: Ruud Zaalberg: The expression of emotion in social situations. The mediating roles of display rules and social motives

2005-8: Catharine Evers: Sex differences in anger expressions. The shaping role of social appraisals

2005-9: Ed Sleebos: The Consequences of Perceized Intra-group Respect: The Effects of Differential Intra-growip Respect on Behavior and Cognition.

2005-10; Jeroen Stouten: Virtue Summons the Fury: Coordination Rutes and Fantriss in Social Dilemwass

2005-11: Irene de Pater: Doing Things Right or Doing the Right Thing: A New Perspective on the Gender Gap in Career Success

2005-12: Rob Nelissen: Guided by Reason, Struck by Entotion: Integrating Motiwational Ex Expectrancy Value Accounts of Beharior

2005-13: Emely de Vet: Testing the Transtheoretical Model: Validity and Applicability for Fruit Jntake 